\title{
Conventional Nanosized Drug Delivery Systems for Cancer Applications
}

\section{Vergallo, Cristian}

Springer International Publishing AG 2021

Vergallo , C , Hafeez , M N , lannotta , D , Santos , H A, D'Avanzo , N , Dini , L , Cilurzo , F , Fresta , M , Di Marzio , L \& Christian , C 2021 , Conventional Nanosized Drug Delivery Systems for Cancer Applications . in F Fontana \& HA Santos (eds), BIO-NANOMEDICINE FOR CANCER THERAPY . Advances in Experimental Medicine and Biology, vol. 1295 , Springer International Publishing AG , Cham , pp. 3-27 . https://doi.org/10.1007/978-3-030-58174-9_1

http://hdl.handle.net/10138/332933

https://doi.org/10.1007/978-3-030-58174-9_1

Downloaded from Helda, University of Helsinki institutional repository.

This is an electronic reprint of the original article.

This reprint may differ from the original in pagination and typographic detail.

Please cite the original version. 


\title{
Conventional Nanosized Drug Delivery Systems for Cancer Applications
}

\author{
Cristian Vergallo, Muhammad Nadeem Hafeez, \\ Dalila lannotta, Hélder A. Santos, Nicola D'Avanzo, \\ Luciana Dini, Felisa Cilurzo, Massimo Fresta, Luisa Di \\ Marzio, and Celia Christian
}

\begin{abstract}
Clinical responses and tolerability of conventional nanocarriers (NCs) are sometimes different from those expected in anticancer therapy. Thus, new smart drug delivery systems (DDSs) with stimuli-responsive properties and novel materials have been developed. Several clinical trials demonstrated that these DDSs have better clinical therapeutic efficacy in the treatment of many cancers than free drugs. Composition of DDSs and their surface properties increase the specific targeting of therapeutics versus cancer cells, without affecting healthy tissues, and thus limiting their toxicity versus unspecific tissues. Herein, an extensive revision of literature on NCs used as DDSs for cancer applications has been performed using the available bibliographic databases.
\end{abstract}

\section{Keywords}

Drug delivery systems $\cdot$ Colloidal nanoparticles $\cdot$ Lipid nanoparticles · Liposomes · Polymeric nanoparticles · Metallic nanoparticles - Carbon nanostructure ·

Nanocrystal · Dendrimers · Hyper-branched polymers · Anticancer therapy $\cdot$ Passive targeting $\cdot$ Active targeting . Aptamers $\cdot$ Therapeutic proteins

Cristian Vergallo and Muhammad Nadeem Hafeez contributed equally with all other contributors.

\section{DDSs Enhancing the Therapeutic Effects of Co-delivered Drugs in Cancer Treatment}

Cancer is the most common disease and leading cause of death, and was responsible for an estimated 9.6 million deaths in 2018. Globally, about 1 in 6 deaths is due to cancer [201]. Chemotherapy represents a therapeutic option for cancer treatment that uses drugs, which are administered by different routes, such as intramuscular injection, intravenous push technique, or intravenous infusion, to kill cancer cells [47]. However, several side effects are associated with chemotherapeutics. They include toxic effects and detrimental metabolic activities for healthy cells; in addition, some cancer cells may not be responsive to anticancer drugs, thus developing resistance [75, 184]. Unfavorable toxicity, development of drug resistance, and limited regime in clinical uses are associated with the anticancer treatment by using single chemotherapeutic drugs [159]. To overcome these drawbacks of chemotherapy, in the past decade, the combination of different chemotherapeutic drugs has gained impressive attention. Multiple anticancer drugs are simultaneously used in order to decrease each drug's toxic side effects on normal cells, while, at the same time, enhancing synergistic and additive effects with high therapeutic efficacy on cancer cells. Several NCs, such as lipid-based NCs, metallic/nonmetallic nanoparticles (NPs), nanoshells, nanorattles, quantum dots (QDs), fullerenes, nanotubes
C. Vergallo $\cdot$ M. N. Hafeez - D. Iannotta $\cdot$ F. Cilurzo $\cdot$ L. Di Marzio C. Christian $(\triangle)$

Department of Pharmacy, University of Chieti-Pescara "G.

d'Annunzio", Chieti, Italy

e-mail: c.celia@unich.it

H. A. Santos

Faculty of Pharmacy, University of Helsinki, Helsinki, Finland
N. D’Avanzo · M. Fresta

Department of Health Sciences, University of Catanzaro "Magna Graecia”, Catanzaro, Italy

L. Dini

Department of Biology and Biotechnology "Charles Darwin", University of Rome "Sapienza", Rome, Italy

CNR Nanotec, Lecce, Italy 
Fig. 1 Nanosized DDSs commonly used to treat different types of cancers

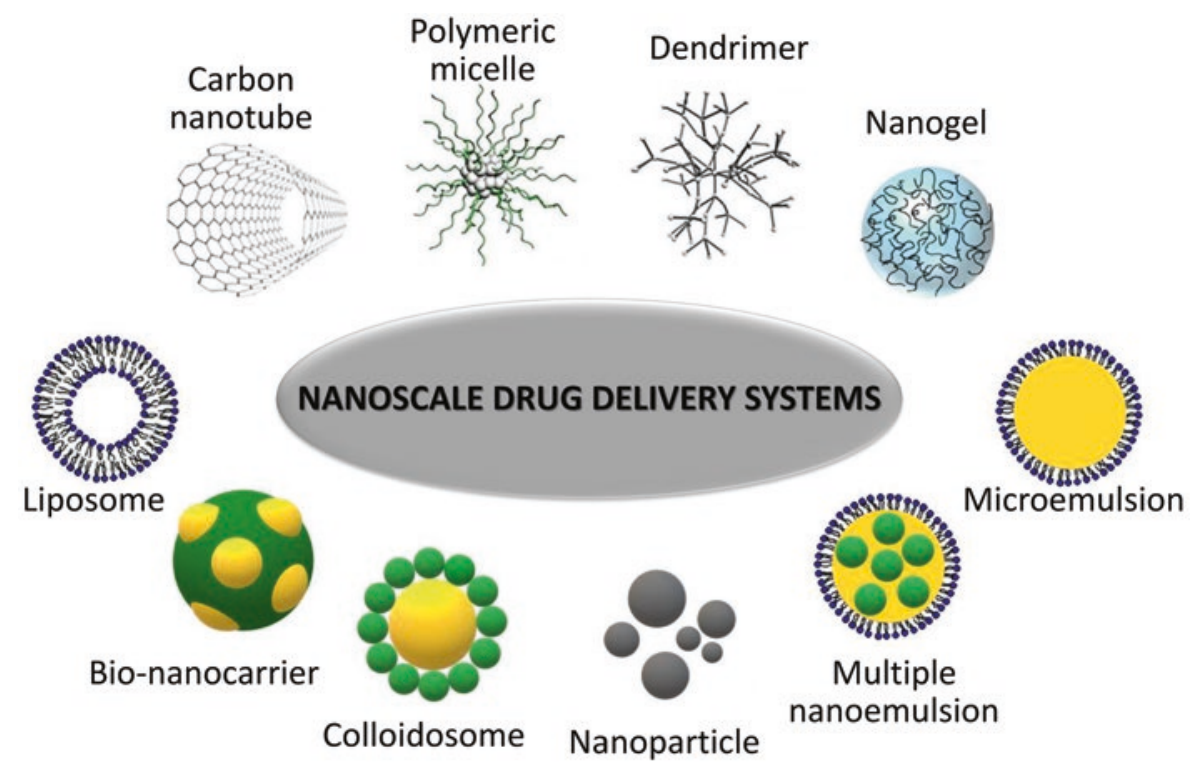

(NTs), carbon nanostructures, monodisperse-nanocrystals, close-packed nanocrystal assemblies, dendrimers, hyperbranched polymers, emulsions, and nanomicelles have been designed and exploited as co-delivery systems to treat different types of cancers. The administration of multiple drugs in drug delivery systems is still prevented by several challenges such as different pharmacokinetic properties and water solubility of the combined drugs, which significantly affect their bio-distribution and blood circulation, the targeting on the tumor site, the bio-accumulation in tissue, and the drug circulation in the blood stream [217, 218].

Examples of nanosized DDSs that have recently been gaining interest from the scientific community and clinicians to enhance the therapeutic effects of co-delivered drugs in combination chemotherapy are shown in Fig. 1. Due to their enhanced bioavailability and blood circulation (e.g., DDSs modified after self-assembling in order to prevent the uptake by the mononuclear phagocyte system), permeability and retention (EPR) effect, nanosized DDSs can target selectively cancer cells [174]. The simultaneous delivery of multiple drugs at targeted tumor tissue is achieved by nanosized DDSs with normalized pharmacokinetics and pharmacodynamics of therapeutic agents. Nanosized DDS-based therapy shows several other advantages over other approaches such as prevention from drug degradation, controlled release of drug, high accumulation rate at the action site, and reduced side effects of drugs on normal body cells [174]. Carrier-free nanodrugs recently emerged as potential generation of nanosized DDSs. Photodynamic therapy (PDT) and photothermal therapy (PTT) have comprehensively been exploited to improve the biosafety and therapeutic efficacy of chemotherapeutic drug treatment of malignant cancers.

The loading of imaging agents into the carrier is another important advantage of nanosized DDSs, resulting in ther- anostic nanosized DDSs, which allow a rapid biodistribution and an easy pharmacokinetic analysis, as well as a real time tracking of drugs and an enhanced performance of delivery process, thus predicting its/their therapeutic effect(s) [202]. Nanosized DDSs are typically developed by loading multiple anticancer drugs within NCs such as polymer micelles, inorganic NPs, and liposomes. Materials for the synthesis of these NCs should be inert and not exert any dangerous side effects. In fact, they have safety properties and need to prevent such events as inflammation and systemic toxicity. It is also worth mentioning that the average loading capacity of nanosized DDSs is relatively low $(<10 \%)$. Therefore, it should be highly desirable to develop alternative nanosized DDSs with high drug loading efficiency [218].

\section{$2 \quad$ Lipid-Based NCs}

Lipid drug conjugates and lipid based NCs are conventional nanosized DDSs used for cancer treatment. Lipid-drug conjugates are drug molecules that have been covalently modified with lipids, showing several advantages such as improved oral bioavailability, enhanced tumor targeting, reduced toxicity, and increased payloads [94]. Conventional lipid-based NCs increase the water solubility of hydrophobic compounds in the blood stream due to the biopharmaceutical properties of the NCs [126]. Lipids render these nanocarriers suitable for drug delivery and therapeutic applications. A common example of lipid-based NCs is liposomes that mainly consist of phospholipids, which are the main components of the biological cell membranes [61]. Polymer-lipid hybrid NPs have been recently introduced as novel DDSs [203]. They are made up of a polymeric core coated by a phospholipid shell and provide some advantages of both polymeric NPs and 
liposomes in the form of a unique shell-core structure [208]. The solid core structure of these NPs provides both structural and mechanical stability, which allows a larger surface area, as well as a narrow size distribution [20]. The outer layer of lipid-based NCs is similar to a cell membrane and provides them with high bio-compatible properties [148]. Their improved drug loading and increased encapsulation efficiency [216] allow the delivery of both hydrophobic and hydrophilic drugs, thus promoting the intracellular uptake of payloads [151].

In a recent study, zinc(II)phthalocyanine (photosensitizer) has been combined with thiophenyl and loaded into a lipid $\mathrm{NC}$ in order to evaluate its therapeutic efficacy toward cancer treated by PDT. The substitution of thiophenyl in zinc(II) phthalocyanine significantly improves its photodynamic responsiveness, thus representing a useful therapeutic tool to treat hepatocellular carcinoma (HCC) [54]. Albano and coworkers [8], in an effort to minimize the total amount of excipients, formulated solid lipid nanosized DDSs by combining cetyl palmitate with two poloxamers, Pluronic-F68 and F127, as inhibitors of glyco-protein efflux pump. When these polymer-lipid NCs were tested as therapeutics for cancer treatment, both high encapsulation efficiency (more than $90 \%$ ) and sustained release of docetaxel were obtained. To address the challenges in the therapeutic efficacy of the topotecan and metformin (metabolic modulator) combination, lipid bilayer-camouflaged mesoporous silica NPs were formulated. In addition, a novel ion pairing-assisted loading procedure was developed by using pamoic acid as an in situ trapping agent. It was demonstrated that pamoic acid increased the hydrophobic properties of metformin and topotecan, thus resulting in high significant payload efficiency (>40 and 32 weight (wt)\%, respectively) in the mesoporous silica NPs and in a controlled release profile. The synergistic cytotoxicity of both drugs, via cell cycle arrest and depolarization of the mitochondrial membrane, was significantly increased toward MDA-MB-231/4 T1 cells compared to the control. Interestingly, the results of preclinical trials showed pharmacokinetic profiles with 4-7 fold higher circulation and 7.5-10 fold higher concentration at the tumor site for these two drugs, when injected using NPs, than the free drugs [15].

The conjugation of some natural bioactive compounds to lipid based NCs improves their bioavailability and water solubility, and it represents the main challenge of this strategy in drug development. A polymer-lipid hybrid $\mathrm{NC}$ was developed by Du et al. [46] to encapsulate the natural compound furanocoumarin. The entrapped compound showed a delayed release compared with the free furanocoumarin. This NC showed similar antitumor efficacy but lower toxicity than the chemotherapeutic anticancer agent doxorubicin (DOX) in a MCF-7 breast tumor model [46].
The combination of diagnostic and therapeutic agents in the same lipid based NCs is an interesting and emerging approach for cancer treatment. Camptothecin (CPT), a liposomal formulation, containing quantum dots, and made up of dimeric CPT glycerophosphorylcholine, significantly inhibits the growth of HepG-2, MCF-7, and HeLa cells, having $62 \mathrm{wt} \%$ loading capacity and stability in physiological fluid [58]. Results demonstrated that the resulting NCs can provide a new strategy for synergy therapy of cancer. Similarly, docosahexaenoic acid and DOX have a longer half-life, a higher tumor uptake ratio, lower toxicity and a higher tumor growth inhibition profile when co-loaded into radiolabeled lipid-based NC-Tc-99 $\mathrm{m}$ and used to treat a breast cancer murine model [63].

Melphalan and miR-181a were also successfully codelivered by lipid NCs for the treatment of retinoblastoma [185]. The co-delivery of regulator protein family B-cell lymphoma 2 (BCL-2) siRNA and epirubicin using lipid NCs showed an increased intracellular uptake and drug release in the tumor microenvironment, thus enhancing the anticancer efficacy as measured by the reduction in cell viability and in the expression of P-glycoprotein [212].

Cell penetrating peptides are another promising approaches in targeted cancer treatment, particularly, after their loading in lipid based NCs. Two types of cell penetrating peptides (linear-peptide RGERPPR, RGE, and cyclicpeptide CRGDRGPDC, cRGD) were used to modify gambogic acid-loaded lipid-based NC, and thus obtain gambogic acid-lipid-based NC-RGE, gambogic acid-lipid-based NC-cRGD, and gambogic acid-lipid-based NC-cRGD/ RGE. The cellular uptakes of these lipidic formulations were qualitatively and quantitatively evaluated swapping gambogic acid for coumarin- 6 as the fluorescent molecule. A high cytotoxicity was obtained for gambogic acid-lipidbased NC-RGE; this result agreed with high intracellular uptake of drug obtained in case of RGE-coumarin 6-lipidbased $\mathrm{NC}$ and the higher tumor growth inhibition resulted with RGE-gambogic acid-lipid-based NC [91].

\section{$3 \quad$ Metallic and Nonmetallic NPs, Nanoshells, Nanorattles, and QDs}

Metal NPs are submicron scale entities made of pure metals (e.g., gold, platinum, silver, titanium, zinc, cerium, iron, and thallium) or their derivatives (e.g., oxides, hydroxides, sulfides, phosphates, fluorides, and chlorides) [85]. Metallic, nonmetallic NPs, nanoshells, nanorattles, and QDs have several applications in the biomedical area, including cancer therapy. Magneto-plasmonic nanohybrid NCs, having a metallic shell of $\mathrm{Au} / \mathrm{Ag}$ coated with $\mathrm{Fe}_{3} \mathrm{O}_{4}$ and poly (comethacrylic acid-butyl methacrylate-co-acrylamide), coloading letrozole as the anticancer drug, are used as 
switchable trimodal temperature/light/pH-triggered and controlled/targeted drug delivery systems for chemo-PTT against MDAMB-231 breast cancer cell. The superparamagnetic internal core provides targeted delivery properties to the plasmonic-magneto-nanohybrid NCs, while phototriggering and photothermal properties depend on the $\mathrm{Au} / \mathrm{Ag}$ shells [85]. Amoli-Diva and co-workers developed bimetallic plasmonic $\mathrm{Ag}-\mathrm{Au}$ and $\mathrm{Au}-\mathrm{Ag}$ (core) NPs. The bimetallic core of NCs is activated by wavelengths to produce heat energy, which not only can increase the surrounding temperature over the upper critical solubility temperature of the polymer to open its valves and promote drug diffusion, but also can kill cancer cells through photo-thermal effects increasing the environment temperature by nearly $18{ }^{\circ} \mathrm{C}$ in $5 \mathrm{~min}$. After radiation [10]. Changes of the composition and structure of bimetallic NPs modify the optical resonances that can be tuned over a broad range of wavelengths (e.g., ultra-violet-visible, UV-VIS, and near-infrared, NIR). Optical resonances in the NIR region referred as the "Tissue Transparency Window," which includes wavelengths between 800 and $1200 \mathrm{~nm}$, are the most important because they can penetrate both water and human tissue [200]. Similarly, $\mathrm{Au}$ (1,7-phen) $\mathrm{Br}_{3}$ loaded $\mathrm{pH}$-responsive superparamagnetic NC (nanogels) demonstrated higher cytotoxicity, better $\mathrm{pH}$-triggered-controlled release and significant inhibition of tumor cells against human cervical cancer HeLa cell lines than free drugs and conventional chemotherapeutics [162]. Another study demonstrated that plasmonic hollow $\mathrm{Au}-\mathrm{Ag}$ nanoshells (tuned to NIR, laser-irradiation), loaded into mesoporous silica, can effectively and efficiently be used to modulate the release of 5-fluorouracil (5-FU) for PTT and prostate cancer therapy [153].

NCs containing metallic and nonmetallic (inorganichydroxyapatite, mesoporous silica and $\mathrm{Fe}_{3} \mathrm{O}_{4}$ ) nanoshells, and having suitable properties for drug delivery, as therapeutics and targeted agents, represent suitable drug delivery systems for therapeutic use. Inorganic NCs are very promising, because they can be used to study release kinetic and pathway activation by fluorescent probes. The fluorescence signal of tracers could be observed simultaneously with drug release, and thus, this strategy provides several benefits to evaluate the therapeutic effects of the nano-DDSs [55]. Compared with green light (490-570 nm), NIR light (650$900 \mathrm{~nm}$ ) is more suitable for biological applications due to several advantages, such as minimum photodamage to biological samples, minimal interference from background autofluorescence, and acceptable tissue penetration [196]. Nanocomposites containing graphene QDs (GQDs), concanavalin $\mathrm{A}$, lectin protein, and $\mathrm{Fe}_{3} \mathrm{O}_{4}$, co-loaded with DOX, were successfully tested for therapeutic/diagnostic applications on HeLa cells lines. The image analysis showed that more than double concentration of DOX was accumulated inside HeLa cells in the presence of an external magnetic field, due to the incorporation of $\mathrm{Fe}_{3} \mathrm{O}_{4}$ in the NC. The cytotoxicity of DOX was $13 \%$ higher than normal cells, thus clearly indicating that these NCs could be used in cancer therapy [50]. DOX targeted delivery is also achieved by using modified GQD-mesoporous silica-NPs with hyaluronic acid for fluorescent imaging [82].

Biosurfactants have been investigated as excipients making NCs by using QDs. They are basically amphipathic molecules having microbial origin that reduce interfacial and surface tension at liquid-solid-gas-interfaces. Bansal and coworkers [16] demonstrated that GQDs NCs conjugated with folic acid (FA) and biosurfactants could be used as therapeutic and diagnostic agents in clinical cancer treatment. In the future, the use of GQD-bioconjugates may allow the detection and treatment of cancer at an early stage. This may increase the life span of cancer patients [16]. Furthermore, black phosphorus QDs are incorporated into liposomes to make DDS with excellent NIR photothermal properties and drug release properties controlled by light. In vitro experiments demonstrate a good biocompatibility and NIR-lightinduced chemo-photothermal antitumor efficiency [74]. Finally, ultra-small WS2 QDs co-loaded with DOX and mesoporous organosilicas NPs demonstrated high potentiality as therapeutics for synergistic chemo-PTT [119].

\section{$4 \quad$ Fullerenes, NTs and Carbon Nanostructures}

Fullerenes may play an important role in clinical oncology because they form complexes with various anticancer drugs such as cisplatin and DOX. Moreover, fullerenes are versatile delivery platforms because they significantly increase in vitro and in vivo therapeutic/cytotoxic effects of drugs, as shown in vivo in Lewis lung carcinoma $[157,158]$. The therapeutic activity of landomycin, an angucycline antibiotic, was also increased using fullerenes as stable and flexible NCs for cancer treatment. Nanocomposites of fullerenes and landomycin show anticancer drug activity in vitro, thus suggesting their potential use in animal models and a future translation in clinics [24]. Water soluble polyhydroxylated fullerenes (PHFs) are functionalized nanomaterials or fullerenols, which can be used as molecular antioxidants and NPs. Compared to fullerenes, PHFs enhanced cellular permeability and solubility and had a lower toxicity [14]. PHFbased NCs conjugated with methotrexate have $\mathrm{pH}$-dependent controlled release of drug, with a significant decrease of $\mathrm{IC}_{50}$ value, increased AUC, half-life, and cell cytotoxicity, and lack of toxicity for erythrocytes [14].

Carbon nanotubes (CNTs) are cylindrical fullerenes, the third allotrope of carbon, first discovered by Sumio Iijima in 1991 [93]. CNTs with a minimum diameter of one nanometer, and having length of several micrometers, are graphene 
sheets rolled in the form of concentric cylinders [51]. According to the number of sheets present in concentric cylinders, CNTs can be divided into two categories: singlewalled-CNTs (SWCNTs) and multi-walled-CNTs (MWCNTs). SWCNTs have length of 20-1000 nm and diameter of $0.4-3.0 \mathrm{~nm}$ and are made up of a single graphene layer wrapped into a hexagonal cylindrical structure [110]. MWCNTs are composed of several single graphene cylinders, having an inner diameter of $1-3 \mathrm{~nm}$, an outer diameter of 2-100 $\mathrm{nm}$ and a length ranging from 1 to several meters [45]. SWCNTs were conjugated with folate and their cell internalization was mediated via folate receptor (FR) pathway thanks to the FA targeting. Results showed that SWCNTfolate $\mathrm{NC}$ was not suitable for receptor-mediated cancer cell targeting. However, due to their needle-like structure, which allows easy cellular penetration, SWCNTs can be used as drug delivery systems of nucleic acid into cells [33]. Furthermore, an enhanced intracellular uptake of anticancer drugs, like CPT, was achieved by magnetic halloysite NTs conjugated with chitosan oligosaccharides and FA [44].

Besides CNTs, Khoee and co-workers successfully demonstrated the use of titanium dioxide NTs in cancer therapy. Titanium dioxide NT-loaded liposomes (cholesterol, soy lecithin, and polyethylene glycol or PEG) can be used for the prolonged delivery of 5-FU in the treatment of HeLa cells [106]. The use of longer MWCNTs was limited due to their cytotoxic effects. However, PEGylated MWCNTs of $\sim 300 \mathrm{~nm}$ were not toxic and allowed a safe delivery of chemotherapeutic drugs, for example, DOX [219]. Carbon dots can be used as $\mathrm{pH}$-responsive fluorescent therapeutic DDSs for DOX in human gastric cancer cells instead of carbon and titanium dioxide NTs. The fluorescent carbon dots enable the optical labeling and tracking of the drug delivery process for at least $48 \mathrm{~h}$. Carboxyl-rich carbon dots have almost no toxicity to human gastric cancer (MGC-803) and human gastric epithelial (GES-1) cells with a cytocompatibility of over $90 \%$ compared to control (untreated) cells [48].

\section{$5 \quad$ Monodisperse Nanocrystals and Close-Packed Nanocrystal Assemblies}

Homologous series of monodisperse nanometer size crystals are known as nanocrystals. They are characterized by narrow size distributed systems in terms of the shape, size, surface chemistry, and internal structure. Probes with different properties (electrical, magnetic, and optical) are combined to characterize and develop consistent structural models of nanocrystal samples.

Several anticancer drugs have limitations to intravenous administration because of their poor aqueous solubility. One recent approach to address this issue is to entrap these compounds into colloidal nanocrystals during the nanocrystal assembling. The cores of nanocrystals and the relatively packed nanocrystals are composed of pure drugs, and they are stabilized with various excipients. Nanocrystals improve the treatment efficacy and decrease the side effects of drugs to healthy cells because they target specifically the tumor tissue thus delivering payloads in bulk. Their small size and high energy surfaces cause a rapid dissolution following administration, which limits the nanocrystal capability to accumulate at tumor site $[67,140]$. A similar problem was observed with several nanocrystal-based anticancer drugs having pharmacokinetic profiles similar to Abraxane or paclitaxel (PTX) NPs [152, 172]. Among several other benefits, such as reduced hypersensitivity and lower neutropenia, Abraxane dissolves rapidly in biological fluids with an insignificant EPR effect. Several strategies have been investigated till date to interfere with the rapid dissolution of nanocrystal-based anticancer drugs [68, 69]. Among these approaches, the layer-by-layer stabilized coating of polyelectrolytes on nanocrystals is one of the few experimental successes reported, even though polyelectrolyte multilayering of nanocrystals drastically affects the dissolution capability of CPT [178], tamoxifen and PTX [3]. However, several other challenges still need to be addressed, such as opsonization and clearance by the mononuclear phagocyte system, accumulation in spleen and liver, and short half-life $[70,90]$. The charged surface of nanocrystals represents one of the causes for the above-mentioned challenges. One possible solution to this challenge comes from masking nanocrystals with PEG, hindering the surface absorption of plasma proteins [150]. PEGylation of polyelectrolyte multilayer nanocrystals has already reported some advantages in vitro, such as slower dissolution than naked NCs, the increased colloidal stability in physiological media, and the lack of toxic effect. Conversely, pharmacokinetic and biodistribution show that these NCs are quickly cleared from the bloodstream and thus accumulated in the mononuclear phagocyte system organs (i.e., liver and spleen). This result may depend on the PEG which modifies the native surface properties of NCs [152].

Closely packed nanocellulose crystals can be successfully linked with chlorotoxin, a polypeptide, using Brønsted acid ionic as a solvent. The resulting close-packed nanocrystals of cellulose and chlorotoxin had significant biocompatibility and enhanced internalization in the U87MG glioblastoma cell line. Thus, this material may be used as DDSs in anticancer therapy [32]. Furthermore, novel PTX nanocrystals were evaluated for their antitumoral efficacy both in vitro and in vivo in comparison with a solution of free drug. Together with a significant lower systemic toxicity, an enhanced accumulation of nanocrystals-PTX was obtained at tumor site in 
MCF-7-bearing mice. Moreover, the cytotoxicity was found to be time- and dose-dependent [118].

\section{Dendrimers and Hyperbranched Polymers}

Highly branched polymers, or dendrimers, were used for the first time in the field of nanotechnology by Vogtle [26]. Dendrimers are well organized 3D nanoscopic macromolecules (5000-500,000 g/mol) characterized by a narrow size distribution. They are distinguished by the number of layers between each cascade (tree like branches), generally known as generations, which possess functional groups at the terminal surface [49]. Their potential interaction with other macromolecules has been widely increased to make them highly functional in terms of host-guest complexes, thus offering wide applications in drug delivery. Herein, we discuss recent updates about dendrimers and hyperbranched polymers in drug delivery, drug conjugation, and their overall toxicity. To avoid any issue like uncontrolled drug release, low efficacy, toxicity, lower half-life, and lower loading capacity associated with NCs, dendrimers represent the solution for the administration of conventional chemotherapeutic drugs. Dendrimers are small in size, and they can penetrate easily in cancer cells. Penetration of dendrimers in deeper layers of 3D spheroid models and tumors depend on their small size (usually below $10 \mathrm{~nm}$ ) and positive charge [28]. Interestingly, DOX-loaded negatively charged poly(amido amine)-2,3dimethylmaleic monoamide (PAMAM-DMA) dendrimers had lower toxicity, enhanced cell membrane permeability and cytotoxicity over cancer cells, and are converted into positively charged PAMAM-DMA when exposed to acidic $\mathrm{pH}$ at the tumor site [31]. The surface of polyamidoamine G4 (PAMAM G4) dendrimers was modified with PEG to reduce the systemic toxic effect of the dendrimer conjugated with FA while increasing nanocomplex targeting. The FA application to PAMAM G4 improved the cell toxicity of PAMAM G4 and 5-FU-loaded PAMAM G4 in high concentrations due to their increased internalization, while PEGylation increased cell viability in a concentration-dependent manner. This nanocomplex had a good cell growth inhibition of HT-29 human colorectal cancer cells, with low side effects on mice myoblast C2C12 normal ones [142].

Graphene-decorated magnetic dendrimers can be used to co-deliver hydrophobic (curcumin) and hydrophilic (e.g., DOX) drugs to MCF-7 cancer cells. To increase the loading capacity of NCs, edges of graphene oxide sheets were decorated with a magnetic-functionalized polyamidoamine dendrimer with hydrazone groups at the end of the polymer, which not only improved the dispersibility and solubility of sheets but also conferred magnetic property to NCs. These $\mathrm{NCs}$ showed a $\mathrm{pH}$-sensitive release for both drugs, and the drug release behavior was also improved by the coimmobilization of payloads [154]. Unimolecular micelles based on PAMAM dendrimers can be used as tumor-selective diagnostic/therapeutic platform owing to their stability, biocompatibility and selectivity. PAMAM dendrimers conjugated with F3, a peptide, and loading DOX exhibit enhanced permeability/internalization into breast cancer cells (e.g., MDA-MB-231) than PAMAM without F3 [207].

\section{$7 \quad$ Emulsions and Micelles}

Emulsions are two-phase mixtures of insoluble liquids in which a "continuous phase" surrounds vesicles of the "dispersed phase." They are stabilized by surfactants to prevent coalescing of the dispersed phase into the macroscopic phase. The continuous phase of an emulsion is generally the aqueous phase, and the drug can be part of this. The stability of emulsions depends upon the diffusion and delivery of drugs from the hydrophobic interior [105]. Microemulsions are transparent oil-in-water systems with a diameter of 100$500 \mathrm{~nm}$ [167] which are widely studied as drug carriers, in order to improve the systemic, controlled, and targeted delivery of anticancer drugs [171]. DOX and phthalocyanine coloaded microemulsions have been incubated with murine breast cancer (4 T1) for $3 \mathrm{~h}$ at various concentrations. Less than $10 \%$ of $4 \mathrm{~T} 1$ viable cells were observed when photodynamic therapy and chemotherapy were combined at a $1.0 \mathrm{~J} \times$ $\mathrm{cm}^{-2}$ laser light dose with $1.0 \mu \mathrm{M}$ phthalocyanine and $0.5 \mu \mathrm{M}$ DOX. Additionally, these microemulsions were stable, had a suitable biocompatibility and improved photophysical properties, as well a significant pharmacokinetics profile, and upregulated 15 and 25 genes related to apoptosis and anticancer activity, respectively. These interesting features make them promising formulations for clinical trials in anticancer therapy by systemic, topical or transdermal administration [29].

Migotto et al. developed cationic bio-adhesive microemulsions for the intraductal administration of C6 ceramide, a sphingolipid that mediates nonapoptotic as well as apoptotic cell death. C6 ceramide concentration necessary to reduce MCF-7 cells viability to $50 \%$ decreased by 4.5 -fold after nanoencapsulation compared to free drug; a further decrease of cell viability (2.6-fold) was obtained when tributyrin (a prodrug of butyric acid) was added as component of the oil phase of the nanocarrier. This approach provides a significant synergism between payloads and microemulsions that are per se therapeutic [138]. Gonzales et al. [77] highlighted the pharmacokinetic benefits of microemulsions used as NCs, by developing fluorescent poly(ADP-ribose)polymerase 1 (PARP1) inhibitor (PARPi-FL), as an imaging agent targeting the nuclear enzyme PARP1 in small cell lung cancer, loaded nanoemulsions. Interestingly, this nanoemul- 
sion is accumulated in H-69 and H-82 small cell lung cancer cell lines, where the contrast agent escapes and is then accumulated inside the cell nuclei [77].

Micelles are colloidal suspensions having a small diameter in the range of 5-100 $\mathrm{nm}$. They gained attention from the scientific community thanks to their potential use in cancer therapy and diagnosis [170]. Along with the ability to deliver partially water-soluble anticancer drugs, micelles display also a significant penetration inside the tissue, site specificity (by attaching specific targeting moieties to the outer surface), and drug stability which contribute to their enhanced therapeutic efficacy [87, 127]. Micellar nanosystems are composed of hydrophilic and hydrophobic components self-assembled into nanosized ellipsoid, spherical, or cylindrical structures. Recently, hybrid stimuli-responsive micelles have been developed either by integrating metal NPs (gold, silver, and iron oxide) inside conventional micelles or by combining polymers and lipids into a single composite [87]. Micelles received scientific interest in the field of drug delivery because their diameter facilitates an easy and effective penetration into tumor cells. For example, PTX encapsulated inside polymeric micelles showed good efficacy in patients with advanced metastatic cancer $[116,175]$. Similarly, DOX/PTX-loaded micelles [41] and DOX conjugated to pluronic [107] were widely used for cancer treatment. A hydrophobic core of micelles obtained by lipid-conjugated PEG can be used to deliver several poor water drugs, such as PTX, tamoxifen, porphyrin [134], CPT [72], and vitamin K3 [139].

\section{Drug Targeting Strategies}

One of the main challenges in cancer treatment is drug biodistribution. Drugs reach healthy tissues, damaging them or causing side effects. Because of this redistribution toward other tissues, there is a lower drug concentration in the tumor site. Thus, new targeting strategies have been developed [195]. NCs can achieve targeted delivery, extend half-life, and reduce the systemic toxicity in chemotherapy [36]. Two kinds of drug targeting are being investigated, passive and active [13]. The passive targeting is based on the EPR effect, by exploiting the presence of abnormal fenestrations belonging to the tumor vasculature (Fig. 2a). On the contrary, active targeting depends on NCs conjugating ligands that bind to a specific receptor overexpressed on the surface of the cancer cell (Fig. 2b).

\section{$9 \quad$ Passive Targeting}

The EPR effect is caused by the need for tumor cells to access nutrient and oxygen rapidly to fuel their exponential growth. Thus, cancer cells stimulate the growth of new blood vessels by activating vascular endothelial growth factor (VEGF) and other growth factors. These newly formed vessels are structurally and anatomically anomalous. In fact, they have a saccular endothelium with very large fenestra-
Fig. 2 Passive (a) and active (b) targeting. Anatomically, tumor micro-vessels are fenestrated, tortuous, and saccular with irregular and discontinuous patterns of interconnection and branching. Passive targeting takes advantage of these unique pathophysiological properties of tumor vessels, allowing nanosized DDS to accumulate into the tumor tissues. In active targeting, nanosized DDS is recognized and bound to target tumor cells through ligand-receptor interactions by the expression of receptors or epitopes on their cell surface. To achieve high specificity, those receptors/epitopes should be highly expressed on tumor cells, but not on normal ones
A

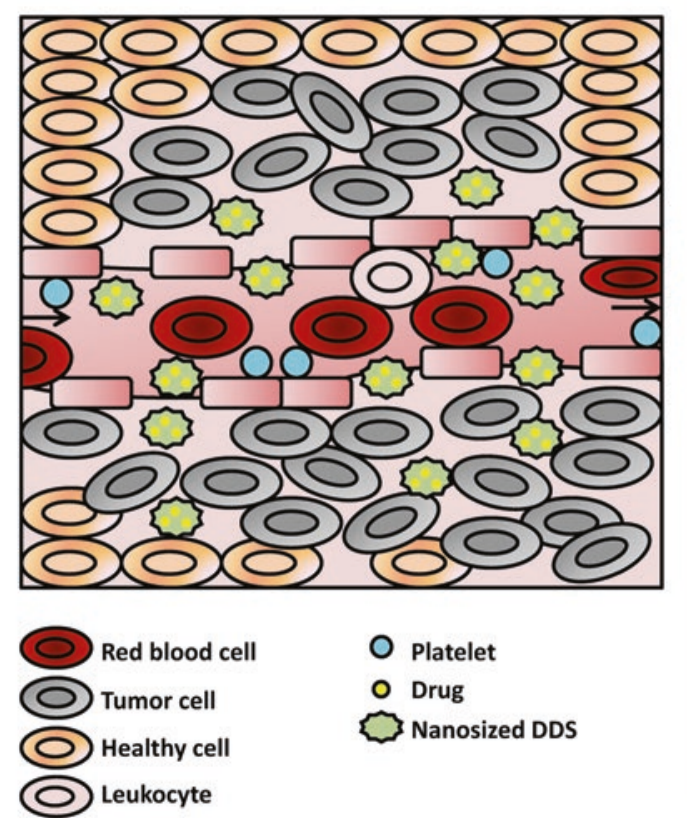

B
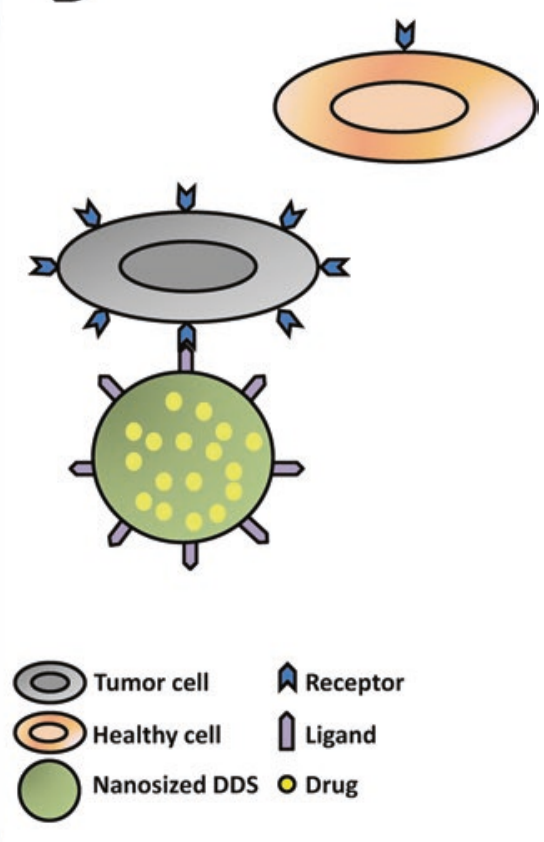
tions, without innervations, smooth musculature and lymphatic drainage, exhibiting altered receptors for Angiotensin II $[21,128]$. Passive targeting exploits dilated endothelium fenestrations, formed because of the abnormal angiogenesis, to accumulate macromolecules/NPs preferentially in the neoplastic tissues [13, 128, 129]; this phenomenon (EPR effect) occurs by a diffusion-mediated transport, allowing a higher accumulation of macromolecules/NPs into tumor cells rather than in healthy ones [128, 129]. However, there is significant heterogeneity of EPR within and between tumor types. Different tumor types have different pore dimensions in the vasculature and that the maximum pore size changes with the location for a given type of tumor (i.e., primary vs. metastases). In addition, there may be differences in the vessel structure within a single tumor type [155]. Heterogeneous consequences are associated with the EPR effect. For example, the central area of metastatic cancers does not exhibit the EPR effect and shows less accumulation of macromolecules than other parts [141]; it facilitates the transport to tumors of nutrients and oxygen that sustain rapid tumor growth [57].

Erythrocytes normally constitute $40-50 \%$ of the total blood volume [190]. Leukocytes and platelets are pushed laterally by the red cells, which tend to accumulate into the vessel core. Thereby, platelets and leukocytes undergo an easy extravasation during inflammation processes [78]. Some NPs have been developed to exploit these underlying phenomena. NPs with the same size of platelets (from ten up to few hundreds of nanometers) flow laterally into the blood vessels and extravasate through the dilated endothelial fenestrations of tumor microvasculature. In addition, the slow blood flow in the tumor microvasculature, due to their anatomical tortuosity, allows NPs to stagnate in the tumor site [13]. The factors exploited in passive targeting, in addition to the anatomical anomalies of the tumor vessels, are the physical characteristics of macromolecules/NPs (e.g., size and shape) as well as the tumor microenvironment, that is, the characteristic of environment around a tumor (e.g., decrease in $\mathrm{pH}$ and increase in temperature) $[17,62]$.

In particular, the tumor microenvironment is characterized by different physicochemical properties in comparison with normal neighboring tissue, such as a lower $\mathrm{pH}$, hyperthermia, and hypoxia [89]. All these differences are useful in the design of stimuli-responsive nanoscale DDSs for cancer therapy.

The responsiveness of DDSs to several stimuli could increase their effectiveness. For this reason, Chitgupi et al. [39] synthesized two liposomal formulations using two different chromophores conjugated to phospholipids, purpurin $(690 \mathrm{~nm})$ or pyropheophorbide $(665 \mathrm{~nm})$. Basic orange and DOX anthraquinones are loaded as single agents into the two liposomal formulations, and they were then mixed together into a single colloidal suspension.
Interestingly, the formulation showed the release of payloads in cancer at a specific wavelength [39]. Fang et al. developed mesoporous carbon NPs modified with hyaluronic acid and GQDs, thus obtaining a dual-responsive targeting drug delivery system capable of providing both in vitro and in vivo synergistic chemo-PTT [56]. Another interesting approach to improve the effectiveness of anticancer DDSs has been proposed recently by Chen et al. and it consists of synthesizing $\mathrm{pH} /$ reduction responsive NPs capable of allowing an efficient delivery and a programed release of drugs in vivo. They developed a core of positively charged DOX-loaded lactobionic acid-chitosanlipoic acid NP which was then coated with a negatively charged dimethylmaleic acid-PEG-chitosan layer to obtain a prolonged circulation time and improve the tumor-targeting effect. NPs targeting the specific tumor tissue were activated by the acidic microenvironment, thus modifying their surface charge from negative to positive with the relative removing of PEG from the surface of NPs. This effect depends on the dimethylmaleic acid-PEG-chitosan layer coating NPs that is $\mathrm{pH}$-responsive to the modification of the microenvironment in tumors. $\mathrm{pH}$-induced responsiveness of NPs caused the rapid release of entrapped DOX and its transfection inside the nuclei where DOX was triggered by the intracellular high concentration of glutathione, and thus activated cellular apoptosis [38].

In order to improve the efficacy of anticancer DDSs, several researches are focusing not only on targeting the general population of cancer cells, but also on others present in the tumor microenvironment [179]. Cancer stem cells (CSCs) are a small subpopulation of cells within tumors with capabilities of self-proliferation, differentiation, and tumorigenicity when transplanted into an animal host [213]. There is a direct correlation between the increase in the number of CSCs and the insurgence of chemoresistance that hinders a successful chemotherapy [122, 213]. Lin et al. recently developed a $\mathrm{pH}$ and glutathione sensitive $\mathrm{NC}$ co-delivering docetaxel and rubone, a miR-34 activator for targeting cancer stem cells, for a selective targeting of CSCs. Therapeutic NCs are specific for the treatment of taxane-resistant prostate cancer and enter the tumor cells by endocytosis pathway. NCs changed their structure and disassembled in the tumor microenvironment after protonation of the $\mathrm{pH}$-responsive diisopropylaminoethanol, making nanocarriers, with the cleavage of the disulfide bond induced by glutathione under acidic condition. This modification results in a fast release of docetaxel and rubone from NCs, thus providing an upregulation of the intracellular miR-34a (rubone-mediated effect), which modulated the expression of proteins involved in chemoresistance, the increased responsiveness of tumor cells versus docetaxel treatment, and a significant inhibition of taxane-resistant tumor progression [122]. Karandish et al. [99] identified and targeted the neuropilin-1 receptor in both 
pancreatic and prostate cancer stem cells with stimuliresponsive PEG-PLA-based polymersomes delivering napabucasin, a stemness inhibitor. In particular, napabucasin-loaded polymerosomes decreased significantly the cell viability of prostate and pancreatic stem cells, compared to controls and cancer cells treated with empty formulation, and reduced the expression of stemness proteins like notch-1 and nanog. These DDSs provide some advantages compared to conventional drug delivery systems in prostate and pancreatic cancer therapy because they deeply penetrate into the tumors, thus releasing the entrapped stemness inhibitor, and killing CSCs [99].

Cancer induces an inflammatory response within the organism that leads to a massive accumulation of macrophages on its onset site (tumor associated macrophages, TAMs). TAMs included two entities: M1 and M2. M1 represents macrophages that follow "classical" activation by interferon- $\gamma$ with either lipopolysaccharide or tumor necrosis factor, whereas M2 represents macrophages that follow "alternative" activation by interleukin 4. M1 macrophages showed a proinflammatory and cytotoxic (antitumoral) function, whereas M2 macrophages are antiinflammatory (protumoral) and promote wound healing [132]. Several studies have shown that the primary tumor growth and the number of metastatic sites can be significantly decreased by decreasing the population of macrophages in tumor tissue, for example, by blocking the recruitment of monocytes or eliminating TAMs already present in the tumor tissue [168]. To this aim, Cao et al. [30]) synthesized PTX-loaded NPs conjugating innovative peptide YI (YINP/PTX) on their surface that can target specifically TAMs and angiogenetic factors. The modified YI peptide increased significantly the specific accumulation of NPs in the tumor tissues [31], thus decreasing the untargeted effect on health tissues and particularly liver [30]. Similarly, Chen et al. [37]) showed that the co-administration of nRGD and lycobetaine-loaded PEGylated liposomes led to an improved tumor penetration and enhanced extravasation [38] which increased the antitumor efficacy against lung carcinoma, likely due to the depletion of TAMs [37].

\subsection{Size and Shape}

Both size and shape play a crucial role for adhesion of some DDSs, like NPs, to tumor endothelium. Even if several NCs for anticancer drugs have been synthesized in spherical forms, many bacteria and viruses display a nonspherical morphology to evade the immune system [102]. Thus, scientists modified the shape of NCs in order to develop smart and novel DDSs. Recent results showed that discoidal NPs are a good compromise between size (few nanometers) and shape, allowing for a greater adhesion surface [146], a better cellular uptake and antitumor efficacy than classical spherical ones [83]. In fact, they can be margined to the blood vessels thus escaping to macrophage uptake and move through the blood stream. Discoidal shape of nanocarriers also generated a circulating second stage of delivering systems that can transport inside other payloads showing potent anticancer activity versus metastatic tumors and improving the rate and long-term survival in vivo of animal engrafted with metastatic tumor cells [206].

\section{$9.2 \mathrm{pH}$}

Several charge-switchable DDSs are developed to improve both the half-life in the bloodstream and their tumor cellular uptake. Due to the accumulation of acid moieties resulting from lower and slower flow of tumor vasculature as well as hypoxic conditions, the tumor microenvironment has a $\mathrm{pH}$ value of about 6.5 versus 7.4 of the normal neighboring tissue [81]. These $\mathrm{pH}$ differences depend on the ion concentrations that modify the microenvironment and the proton concentration, thus activating some mechanisms that can modulate the internalization and uptake DDSs by different pathways like endocytosis. In particular, protons entry into the endosome, by means of a mechanism called "proton sponge," leads to osmotic swelling and finally to endosome rupture, thus releasing the NCs and its payload into the cytoplasm. Other DDSs have been developed by using organic, inorganic, and hybrid $\mathrm{pH}$-sensitive materials. The easy rupture of some bonds at low $\mathrm{pH}$ values allows the drug release at the tumor site $[19,124$, 193]. Recently, a novel polymeric micelle-decorated $\mathrm{Fe}_{3} \mathrm{O}_{4} /$ $\mathrm{Au}$ core-shell NP has been designed for $\mathrm{pH}$-responsive intracellular co-delivery of 6-mercaptopurine and DOX [75].

The main disadvantage of using $\mathrm{pH}$-sensitive DDSs is that they act in a specific $\mathrm{pH}$ range. However, some $\mathrm{pH}-$ sensitive DDSs have been translated from bench to clinical application and have been approved by the US Food and Drug Administration (FDA) for treatment of various cancerous diseases [124].

\subsection{Temperature}

The temperature in different body districts varies according to different factors such as the blood flow and the thermal energy generated by cellular metabolism. Due to their higher metabolic activity and blood flow compared to the surrounding healthy tissue, an increase in the temperature occurs in the tumor tissue $[60,98,100,166]$. Thus, this increase of temperature at the tumor site could be used by thermoresponsive DDSs. Among the many different stimulisensitive delivery systems, temperature-sensitive DDSs offer great potential over their counterparts due to the versatility in their design, the tunability of phase transition temperatures, the passive targeting ability, and in situ phase transitions. In an effort to further control existing temperature-responsive systems, current innovative applications have combined tem- 
perature with other stimuli such as $\mathrm{pH}$ and light. The result has been the development of highly sophisticated systems, which demonstrate suitable control over drug release and represent huge advances in biomedical research [23]. For example, sulfonamide and hydroethylacrylate monomers have been polymerized with poly( $\mathrm{N}$-isopropylacrylamide) to combine $\mathrm{pH}$ and temperature stimuli and thus develop injectable drug delivery systems that do not undergo phase transitions at the lower value of critical solution temperatures in physiological condition [133, 177]. Bikram et al. [22] have developed composite hydrogels of silica-gold nanoshells and poly(N-isopropylacrylamide) co-polymerized with acrylamide to form the photothermal modulated drug delivery system in which NIR light can be used to induce the collapse of the polymeric matrix containing payloads used as drug candidate model [22].

\section{Active Targeting}

Advances in nanobiotechnology and targeting strategy could improve the delivery of therapeutic molecules into cancer cells, thus improving the treatment effectiveness with minimal side effects on healthy cells [189].

Active targeting uses specific ligands to bind receptors overexpressed in tumor tissues. In literature, many ligands have been used for active targeting. They include peptides (e.g., octreotide, asparagine-glycine-arginine, cyclic peptide iRGD (CCRGDKGPDC) and C-end Rule, or CendR, motif peptide tLyP1), proteins (e.g., transferrin, growth factors, antibodies, and antibody fragments), mono- (e.g., galactose) and polysaccharides, glycosaminoglycans (e.g., hyaluronic acid), aptamers, and other molecules (e.g., FA, bisphosphonates, and biotin) [34, 64, 104, 164, 188]. These ligands are conjugated to NPs loaded with the drugs of interest, in order to release higher amounts of payloads in cancer cells rather than in healthy tissues. The choice of specific ligands to bind the appropriate receptors is very important for the right active targeting [13]. Very useful receptors are epidermal growth factor receptor (EGFR) and folate receptor (FR), which are overexpressed in most cancer cells [120, 147]. Active targeting has several advantages including increasing the drug dosage that is delivered to the tumor tissue with the resulting lower systemic administration, and the higher therapeutic efficacy [113, 194].

Targeting agents are conjugated to the DDS surface, thus increasing their interaction with specific cancer cells and tissues. In particular, many papers demonstrated the targeting cancer cells-overexpressed specific tumor receptors and accumulation of drugs inside cancer cells. However, strategies preserving healthy cells and tissues from the toxicity of drug targeting need to be addressed and are still one of the major challenges in pharmaceutical field [33]. Below, we discuss the main targeting agents that are conjugated to innovative DDSs and provided a smart and specific targeting for anticancer therapy (Fig. 3).

\subsection{Peptides and Proteins}

Peptides can recognize and bind receptors overexpressed by cancer cells with high affinity. They are poorly immunogenic and quickly excreted via liver and kidneys. Their synthesis is very simple, but it is quite expensive. In addition, peptides are easily exposed to the action of the peptidase enzyme that cleaves and inactivates these macromolecules, thus altering their biological effects.

The blockade of integrin signaling has been demonstrated to be efficient in inhibiting tumor growth, angiogenesis, and metastasis. Among all the integrins, $\alpha v \beta_{3}$ seems to play a fundamental role during tumor angiogenesis. Integrin $\alpha v \beta_{3}$ is highly expressed on activated endothelial cells, new-born vessels as well as some tumor cells, but it is not present in the other endothelial cells and most normal tissues and organs [125].

iRGD is a prototypic tumor-specific tissue-penetrating peptide (a 9-amino acid cyclic peptide with sequence CRGDKGPDC), which delivers drugs deeply into the extravascular tumor tissue (Fig. 3b). The pathway triggered by iRGD can be used for the enhanced transport of anticancer drugs administered as combination into the tumors. Indeed, Yang et al. [207]) had higher 4 T1 cells cellular uptake in vitro [209, 210] as well as higher penetration and accumulation of legumain-responsive aggregate gold NPs in breast tumor in vivo when these NPs were co-administered with iRGD [209].

Moreover, the cyclic peptide iRGD has been well established as a tumor-homing and tumor-penetrating peptide. iRGD initially binds the $\alpha v$ integrins expressed on tumor endothelial cells. Subsequently, the cleavage by a protease and the bond with neuropilin-1 allows its internalization $[187,188]$. The identity of the iRGD processing protease(s) is unknown [27]. Song et al. [180] reported a DOX loaded liposomes modified with a "tadpole"-like peptide, which was obtained by conjugating covalently the alanine-alanineasparagine "tail" residual to the cyclic tumor homing peptide iRGD to afford nRGD (multi-target peptide consisting of alanine-alanine-asparagine and cyclic iRGD). This DOX loaded liposomes modified with nRGD showed excellent antitumor efficacy in $4 \mathrm{~T} 1$ breast cancer mice than other formulations tested (i.e., free DOX, DOX-loaded PEGylated liposomes, DOX-loaded PEGylated liposomes and modified with iRGD, and DOX-loaded PEGylated liposomes and modified with nRGD), interacting with tumor vascular endothelial cells to achieve efficient tumor penetration, and modulate tumor microenvironment with depletion of tumor associated macrophages [180]. 

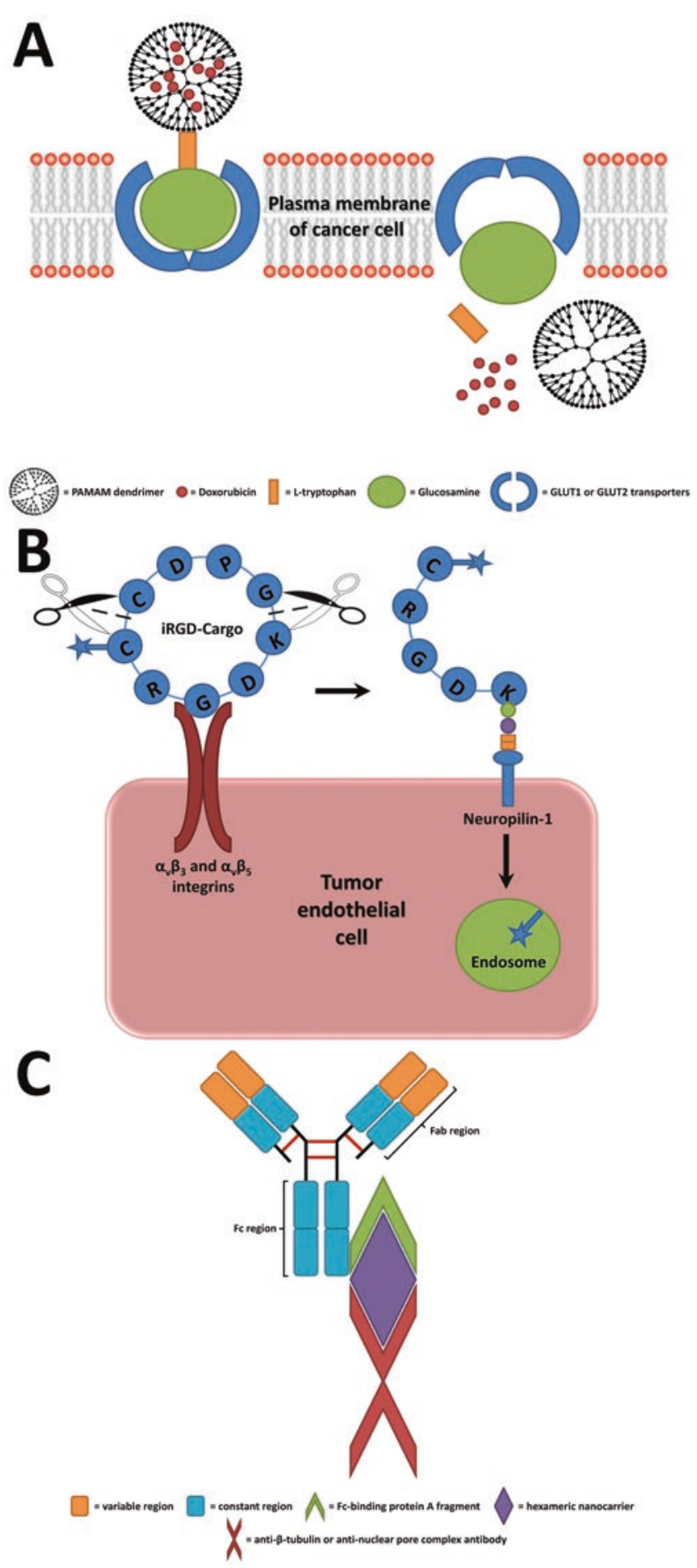

Fig. 3 New generation of DDSs for the enhanced transport of cancer drugs into tumors. (a) GLUTs mediated delivery of anticancer drugs. PAMAM dendrimers modified with L-tryptophan and GlcN permit higher drug loading and the use of GLUT transporters. This DDS allows the DOX release with significant higher cytotoxicity than PAMAM [112]. (b) Drug delivery into extravascular tumor tissue by the prototypic tumorspecific tissue-penetrating peptide CRGDKGPDC (iRGD). iRGD peptide homes and penetrates tumors through a three-step process: (1) the RGD sequence motif mediates the binding to $\alpha v \beta_{3}$ and $\alpha v \beta_{5}$ integrins expressed on tumor endothelial cells; (2) upon $\alpha v$ binding, a protease cleavage event is activated, revealing the c-terminal CendR motif of the peptide; (3) the peptide, by its CendR motif, binds neuropilin-1, thus activating the endocytotic/exocytotic transport pathway [187, 188] (C) Delivering of functional antibodies to the cytosol as intracellular therapeutics without cytotoxicity by Hex NC. This NC has been generated by combining a $\alpha$-helical peptide that self-assembles into a hexameric coiled-coil bundle with an Fc-binding protein A fragment. An anti $\beta$-tubulin or antinuclear pore complex antibody as cargo allows the intracellular delivery of antibodies. The addition of an endosomolytic motif conjugated to the core improves this delivery [121] 
Proteins, such as transferrin and EGF, are also used for active targeting. Transferrin is the major serum glycoprotein which transports iron into cells by receptor-mediated endocytosis, also called clathrin-mediated endocytosis [43]. Since cancer cells have higher transferrin receptor 1 expression than normal cells, this receptor represents a potential targeting to treat cancers by transferrin-conjugated DDSs [173]. However, the administration of elevated doses of transferrinconjugated DDSs may also cause the death of healthy cells or cells with high proliferative activity [104]. Like transferrin receptor 1, also EGFR is overexpressed in human malignancies (e.g., carcinomas), and its activity enhances tumor growth, invasion, and metastasis [144]. The advantage of using EGF as the targeting moiety is that this can promote the transport of the drug to the nuclear level through an endocytic pathway in highly proliferating cells [34]. In addition, the internalization of DDSs conjugated to EGF is more rapid than those conjugated with other proteins, such as antibodies [164]. However, the use of this ligand may promote cell division because EGF is mitogenic [131, 191]. Antibodies are other ligands used for active targeting. The NC is bound to an antibody in the heavy chains portion, so that the two variable light chain endings can bind a specific receptor expressed on the tumoral cell surface [13]. This allows for a directional targeting effect and a controlled release of the drug simultaneously. V-erb-b2 erythroblastic leukemia viral oncogene homolog 2 (ErbB2) is a growth factor receptor overexpressed in 20-30\% of human breast carcinomas as well as other adenocarcinomas. ErbB2 F5-scFv antibody was conjugated to DOX loaded into PEGylated liposomes. These F5-scFv targeted liposomes had antitumor activity and produced significant reduction of the tumor size in xenografted mice compared to nontargeted liposomes loading DOX, Doxil® [143]. Anti VEGF monoclonal antibodies and other VEGF inhibitors block the growth of several tumor cell lines in nude mice as previously reported [64]. Clinical trials with VEGF inhibitors in a variety of malignancies are still ongoing. In particular, a humanized anti VEGF monoclonal antibody (bevacizumab; Avastin $®$ ) has been approved by the FDA as a first-line treatment for metastatic colorectal cancer in combination with chemotherapy. VEGF is also implicated in intraocular neovascularization associated with diabetic retinopathy and age-related macular degeneration [64]. Other examples of new targeted DDSs using antibodies are further reported in the Sect. 19. However, targeted DDSs present also some disadvantages. The therapeutic effect could be altered if the bound DDS-antibody is not degraded correctly. Moreover, the antibody binding ability could be decreased if the fractions involved in this bond (antibody and or antigen) are sterically hampered by the presence of the nanocarrier. Finally, the immunoconjugate DDS-antibody could trigger an immune response in vivo by the organism [109, 163].

\subsection{Aptamers}

Aptamers are short single-stranded DNA or RNA oligonucleotides that can selectively bind to small molecular ligands or protein targets with high affinity and specificity, by acquiring unique three-dimensional structures as previously reported [101]; they have the advantage of being highly specific, relatively small, nonimmunogenic and can be easily stabilized by chemical modifications, thus allowing expansion of their diagnostic and therapeutic potential [101]. They could be conjugated to DDSs by a covalent bond to obtain a high affinity as well as a low immunogenic targeting. In addition, they are stable at different $\mathrm{pH}$ and temperature ranges, easily synthesizable, modifiable and excretable by kidneys [114]. However, because aptamers are composed of DNA or RNA oligonucleotides, they are rapidly degraded by exo- and endonucleases [176], thus the half-life of unmodified nucleotide aptamers in blood can be as short as $2 \mathrm{~min}$. [80]. This issue has been solved through PEGylation. Dassie et al. [42] demonstrated that the RNA aptamer A9g functions as a smart drug for metastatic prostate cancer by inhibiting the enzymatic activity of the prostate-specific membrane antigen. A9g was safe in vivo and not immunogenic for human cells. Pharmacokinetic and biodistribution studies in mice have confirmed the targeting specificity and absence of nonspecific on/off-target effects [42]. Since their excellent properties, different aptamers conjugated with DDSs have been developed for cancer therapy. Sgc8c aptamer-DOX conjugates were found cytotoxic for leukemic cells expressing the protein tyrosine kinase 7 [92]. DOX was covalently conjugated with the AS1411 DNA aptamer, which targets plasma membrane nucleolin, a protein that is overexpressed in many cancer cells [115].

\subsection{Other Molecules}

Several low molecular weight ligand interactions with receptors are used as targeting moieties for DDSs. Among those ligands, folate is often used to target the FR because of its high expression level in most cancer cells compared to healthy cells and tissues. Folate is a very small ligand and has high affinity for its receptor; moreover, it can be easily conjugated with DDSs loading different payloads, and it is cheap, biocompatible, and safe [66].

\section{Combined Targeting}

The dual targeting of DDSs to tumors by different mechanisms could represent a promising translational approach for anticancer treatment. PEG and its derivatives are used as polymeric materials to synthesize NCs that have both active 
and passive targeting properties; nowadays, poly(lactic acid) (PLA), PEG, or their combinations are the most used macromolecules to synthesize therapeutic and targeted NPs [95]. PEG is widely used to prevent the opsonization of NPs after their injection in the blood stream. Opsonins are the proteins involved in this process, and interact with the hydrophobic components of NPs, leading to their flagging as "not self" material. NPs flagged by opsonins are then captured by macrophages forming the mononuclear phagocyte system, and, at the end, eliminated from the body. PEGylated NPs are not recognized by opsonins. Thus, their recognition and uptake by the mononuclear phagocyte system is reduced. Consequently, the circulation half-life of various types of NPs, such as polymer-based NPs and hybrid NPs, is widely increased [79, 117, 145]. However, PEG-related immunological issues have received considerable attention. Antibodies against PEG may limit the therapeutic efficacy and/or reduce tolerance of PEG-asparaginase in patients with acute lymphoblastic leukemia and of PEGloticase in patients with chronic gout. In particular, anti PEG immune reaction occurred in 22-25\% of healthy volunteers and anti PEG antibodies are present in their blood, compared with a very low $0.2 \%$ of anti PEG antibody immune reactions that occurred in the two decades before. This increase may be due to an improvement of the limit of detection of antibodies during the years and to greater exposure to PEG and PEGcontaining compounds in cosmetics, pharmaceuticals, and processed food products. Thus, patients should be prescreened and monitored for anti PEG prior to and throughout the course of treatment with a PEGylated compound [73].

\subsection{Anticancer Nanosized DDSs on Market}

To overcome chemotherapy-associated drawbacks (see Sect. 1), great progress has been made in the field of anticancer nanomedicine, which led to the development of smart and novel DDSs with characteristics including size not exceeding $200 \mathrm{~nm}$ and versatile architecture [2]. The main advantage provided from these nanosized DDSs is the increase of the surface area [53]. To date, several liposomal- and NP-based DDSs for cancer treatment have been approved in different countries. In 1995, Doxil ${ }^{\circledR}$, lipo-DOX, was the first US-approved (by FDA) nanodrug. Its corresponding equivalent drug approved in the E.U. was Caelyx ${ }^{\circledR}$, and both are used for the treatment of Kaposi's sarcoma, breast, multiple myeloma, and ovarian neoplasms, and had a better toxicity profile than free DOX hydrochloride. In 1996, FDA approved DaunoXome $^{\circledR}$, another lipo-DOX formulation, used in cancers and HIV-associated Kaposi's sarcoma. Recently, other liposomal-based drugs have been approved both in the E.U. and USA. In 2009, Mepact $^{\circledR}$, liposomal mifamurtide, was marketed as a powder which is resuspended into a suspen- sion for infusion to treat osteosarcoma. In 2012, liposomal vincristine sulfate, commercially available as Marqibo ${ }^{\circledR}$, was marketed to treat Philadelphia chromosome-negative chronic myelogenous leukemia. In 2015, Onivyde ${ }^{\circledR}$, lipo-irinotecan, was marked as a chemotherapeutic agent to treat metastatic pancreatic cancer [2]. Furthermore, several DDSs are currently under clinical trials, such as AS15 (liposomal adjuvant AS15 containing monophosphoryl lipid-A, Quillaja Saponaria plant extract QS21 and CpG oligodeoxynucleotide, phase II trial), SP1-077 (cisplatin-loaded PEG-liposome treating lung, head, and neck cancers, phase II trial) and ALN-VSP (lipid-based NPs containing VEGF-A and kinesin spindle protein siRNAs, developed for the treatment of advanced cancer and liver metastases, phase I trial) [2]. NP-based DDSs currently in the market include Abraxane ${ }^{\circledR}$, PTX-loaded albumin NPs, approved in 2005 for non-small cell lung carcinoma and pancreatic neoplasm therapy, and Zevalin $^{\circledR}$, a monoclonal antibody radio-immunotherapy treatment for relapsed or refractory, low grade or transformed B-cell non-Hodgkin's lymphoma, approved in 2004 [59].

\section{Efficacy and Side Effects}

DDSs can increase the therapeutic efficacy of several anticancer drugs, by decreasing the drug side effects, and increasing the patient's compliance [123]. Compared to free drugs, DDSs promote drug accumulation preferentially in the disease area. To enhance the DDS effectiveness, many strategies have been developed to increase their targeting toward specific sites. The conjugation of targeting moieties on the surface of NCs is one of the strategies used to increase the accumulation. Recently Ai et al. [4] synthesized integrin targeted iRGD-heparin NPs loading cis-diamminedichloroplatinum(II) to clinically treat gastric cancer. These NPs showed a significant antitumor activity of payload without the weight loss or liver and kidney damage occurring in the nude mice, bearing MKN-45P tumors, that are treated with free diamminedichloroplatinum(II) or untargeted heparin NPs [4].

Interestingly, drugs can become sensible to endogenous and/or exogenous stimuli [161]. Daga et al. [40] developed glutathione-responsive cyclodextrin nanosponge loaded DOX for anticancer therapy. These nanosponges were able to release DOX preferentially in cells having high glutathione content, such as DU145 prostate and HCT116 colon cancer cells, as well as to inhibit the growth of DU145 xenograft tumors more than DOX [40]. Similarly, Argenziano et al. [11] generated strigolactones-loaded glutathione/pHresponsive nanosponges to selectively deliver strigolactones to prostate cancer cells and increase their therapeutic efficacy [11]. Moreover, the therapeutic effectiveness of DDSs can be increased also by co-delivering various anticancer drugs simultaneously (see Sect. 1). 
NC protects drugs from degradation after systemic injection, thus increasing their half-time, leading to less frequent administrations, improving patients' compliance and decreasing healthcare costs [75, 130, 205]. Unfortunately, DDSs exhibit some detrimental reactions/side effects strongly correlated with their physicochemical properties and material composition [135]. The side effects include complement activation, which can cause both a rapid drug clearance and a pseudo-allergic reaction in patients [9], and blood adverse reactions such as hemolysis, thrombogenicity, and/or vascular oxidative stress that result in inflammatory responses $[135,137]$.

\section{Physicochemical Properties}

Physicochemical properties, such as size, shape, surface chemistry, and polydispersity index (PDI), are fundamental parameters affecting the performance of DDSs (Fig. 4). In particular, they influence primary biopharmaceutical properties such as biodistribution, pharmacokinetic, therapeutic versus side effects of drugs. DDSs can be manipulated and change their physicochemical properties to obtain a personalized medicine for cancer therapy [220]. As a result, DDSs increased the effectiveness of cancer treatments compared to free drugs.

\subsection{Size and Shape}

Size is directly proportional to the surface area of DDSs interacting with the external environment after administration. Different studies, most of them involving NPs, are currently ongoing to study the mechanisms of DDS cell internalization. The cellular uptake of NPs is a size-dependent

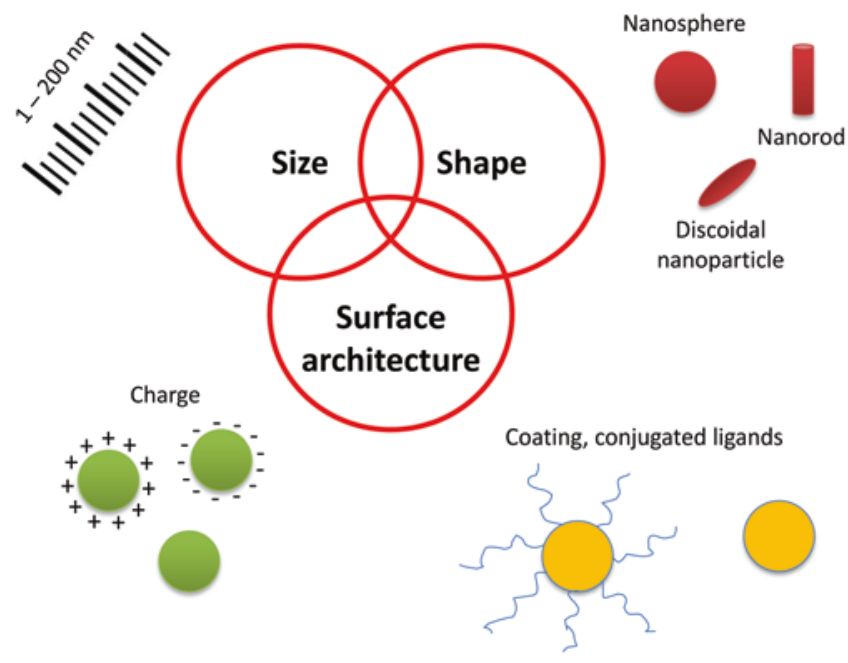

Fig. 4 Main physicochemical properties affecting biopharmaceutics of DDSs process that starts from the interaction between NPs' ligands and the receptors expressed on the cell surface. These interactions provide enough energy to bypass the phospholipid bilayer forming the plasma membrane. Thermodynamically, a NP having a size between 40 and $50 \mathrm{~nm}$ can interact and bind receptors to successfully produce membrane wrapping. NPs of $\sim 50 \mathrm{~nm}$ bind many receptors and their uptake is limited by the redistribution of receptors on the cell membrane via diffusion to compensate for local depletion. NPs larger than $50 \mathrm{~nm}$ bind with high affinity to a great number of receptors and may limit the further binding of additional NPs [7]. Size is also a crucial property that allows NPs to extravasate from the abnormal fenestrations belonging to the tumor vasculature. This extravasation is possible only for DDS with dimensions smaller than $200 \mathrm{~nm}$ [108]. Furthermore, the NP size strongly affects the type and the amount of proteins adsorbed on their surface after administration in the blood stream. Plasma proteins, such as serum albumin, complements, immunoglobulins and apolipoproteins, are adsorbed onto the NP surface and form a "protein corona." Basically, there are hard and soft corona proteins that cover the surface of NPs. The protein corona phenomenon can modify the surface properties and targeting on nanoparticles, thus decreasing their specific targeting and modifying their therapeutic response in preclinical and clinical studies [5, 86]. Protein corona also affects the physiological property of DDSs, their circulation lifetime, accumulation, toxicity, cellular uptake, and agglomeration [111, 220].

Shape is another crucial characteristic that strongly affects the fate of DDSs because it is involved in several processes occurring after administration, such as biodistribution, macrophage phagocytosis, cell uptake, and targeting [220]. In fact, the shape of NPs can affect their metabolic pathway and the interaction with the cell surface. Spherical shape is better taken up by macrophages and cleared by renal filtration, while discoidal or nonspherical NPs long-circulate inside the blood stream thus avoiding the macrophage uptake and the clearance [103]. This effect depends on the different marginalization of NPs and their distribution in the blood stream. Moreover, opsonins are better adsorbed on the surface of spherical NPs than discoidal or nonspherical one due to their curvature radius that facilitates this interaction and modify the relative surface properties of NPs [86].

\subsection{Surface Chemistry}

The surface charge plays a crucial role in protein adsorption, which in turn affects the pharmacokinetics and biodistribution of NCs [25]. Circulation time of NCs in blood vessels is highly dependent on charge. For example, highly cationic NPs are rapidly cleared from circulation greater than highly anionic NPs. In contrast, neutral NPs, as well as those with a 
slight negative charge, show significantly prolonged circulating half-lives [12]. Surface charge affects both internalization and accumulation of NCs in the tumor cells [215]. Conversely to neutral NPs, positively charged NPs show a greater rate of interaction with cancer cells in response to the electrostatic force between the NP surface and the plasma membrane [71, 97]. Xiao et al. [204] showed that the amount of PEG-oligocholic acid NPs accumulated in the tumor was inversely correlated with their surface charge densities (either positive or negative) [204]. Particularly, they demonstrated that the highest tumor accumulation could be achieved by rendering the surface slightly negative. Although it is not good enough for long circulation and tumor accumulation, the positive surface charge improved the tumor extravasation and penetration. For example, He et al. demonstrated that the surface charge density had different impacts on tumor accumulation for negatively and positively charged NPs [88]. Higher surface charge densities caused lower tumor accumulation for negatively charged NPs; however, for positively charged particles, NPs with higher charge densities showed higher accumulation in the tumor [88]. NPs with a higher positive charge density could more efficiently escape from the interstitial space and be internalized by tumor cells and the associated endothelium [220].

\section{Health Costs of DDSs in Cancer Therapy}

As of 2018, almost 250 therapeutic DDSs were available for clinical use [53], and the perspective assumes the double for 2019 [18]. A small part of these DDSs has been already marketed, whereas all the others are still waiting for approval [1]. On June 2018, US FDA adopted a specific policy for improving their approval [169], whereas European Medicines Agency (EMA) formed a group composed by experts in the field of nanotherapeutics to better outline and review guidelines [65]. Shortening the approval times for new DDSs for cancer therapy could have a net effect on saving public health costs. Targeted drug delivery and conventional medical management have shown to promote a decrease in healthcare use and costs for cancer patients in comparison to conventional medicines. Stearns et al. [182] conducted a retrospective economic evaluation using score-matched analysis by MarketScan commercial claims data on beneficiaries receiving targeted drug delivery and/or conventional medicines for cancer therapy. The survey examined the period from January 1,2009 , to September 30, 2015. In this economic evaluation, involving 536 patients from a large US payer database, the use of targeted drug delivery and conventional medicines versus conventional medicines alone was associated with a significant overall cost savings of $\$ 63,498$ in the 12 months after the index date and with significantly fewer inpatient visits, shorter hospitalization, and fewer emergency department visits. In the short term and with respect to conventional medical management alone, the use of targeted drug delivery and conventional medicines was associated with significantly higher outpatient and lower inpatient costs [182].

\section{Receptors}

Receptor-targeted drug delivery has been extensively explored to target/treat cancer cells. Several studies about drug targeting involved the high-affinity receptor for the vitamin FA, also known as FR. The high affinity of the FR toward FA has been also recently confirmed by Kumar et al. [112]. They evaluated the targeted delivery of gum kondagogu-capped gold NPs coupled with FA in both FR positive (MCF-7) and FR negative (A549) cancer cells. A great affinity of these NPs was found toward FR positive cells [112]. In some cases, FA is used to improve the passive targeting of DDS by introducing an active targeting too, thus increasing its effectiveness [189]. Ak et al. [6] synthesized DOX-loaded and glucose/gluconic acidcoated magnetically responsive NPs. Erythrocyte membrane vesicles were used as coating, while folate ligand was anchored to their surface to target the FR. Interestingly, these FR-targeted NCs showed more cytotoxicity against ovarian cancer cells than nontargeted ones [6]. Drug delivery by FR has also proved some effects for other chemotherapeutic agents for the treatment of various cancers, such as PTX and gemcitabine (GEM). In the cytotoxicity study carried out by Erdoğar et al. [52] it was found that the breast cancer cells were more sensitive to cytotoxic effects of PTX delivered by folate-conjugated cyclodextrin NPs than PTX in Cremophor EL $®$ solution [52]. The analysis of parameters that facilitates direct drug delivery into the cancer cells is very important to consequently select a suitable pathway of drug penetration. For example, singlewall carbon nanotubes (SWNTs) below $450 \mathrm{~nm}$ could be used as DDSs to transfect nucleic acids into the cells due to direct cell penetration based on their needle-like structure, whereas SWNTs over $450 \mathrm{~nm}$ are suitable to target the cells through the FR [33]. The systemic use of free GEM is restricted because of its poor physicochemical properties and nonspecific drug delivery, resulting in dose-dependent adverse effects. With respect to free GEM, fucose-conjugated GO nanosheets provided high loading efficiency of GEM, which was optimized by increasing the drug concentration and maintaining a constant fucose-conjugated GO nanosheets concentration. This strategy provided a sustained $\mathrm{pH}$-dependent drug release, and an affective targeting of GEM toward both human breast cancer MDA-MB-231 cells and human lung cancer A549 cells overexpressing fucose-receptor [84]. The conjugation of several ligands in the same NCs could improve the responsiveness of tumor cells versus these formulations. Wang et al. 
[199] synthesized synergetic targeted liposomes which conjugated on their surface both a tumor identification ligand, the antiestrogen receptor (ER) antibody, and an immune targeting ligand, that was the critical segment of Cluster of differentiation 47 (CD47), called self-peptide (SP) [198]. The anti ER antibody recognizes and binds ER-positive breast cancer tissues in a specific way, while SP activates the CD 47-Signal regulatory protein $\alpha(\mathrm{CD} 47-\mathrm{SIRP} \alpha)$ immune response, thus decreasing the phagocytosis of NPs by macrophages. Both the enhanced targeting ability and antiphagocytosis properties improve the tumor uptake of the NCs and prevent their immune clearance [199]. Yang et al. [207, 209, 210] synthesized DOXloaded micelles made up of hyaluronic acid conjugated with FA via a reduction-sensitive disulfide linkage to form an amphiphilic polymer (HA-ss-FA). Cellular uptake and in vivo biodistribution studies showed that these DOX-loaded micelles targeted specifically tumor tissues and enhanced significantly cellular uptake by CD44 receptor-mediated endocytosis. The cellular uptake of DOX in CD44-positve A549 cells was 1.6fold more than that in CD44-negative ones. In addition, the biodistribution of HA-ss-FA micelles showed promising results in in vivo tumor targeting in human (HCC-LM3)bearing nude mice [210].

\section{Transporters}

Cells have a membrane with different protein transporters that work as channels or pumps driving in and out the molecules according to a specific gradient. The protein transporters protect the internal cell environment and keep its homeostasis for salts, nutrients, and proteins within a physiological range. These protein transporters can allow the specific delivery of anticancer drugs into the cell, thus bypassing the high selectivity of the cell membrane. Glucosamine (2-amino-2-deoxy-D-glucose, GlcN) and N-acetyl glucosamine (GlcNAc) are amino monosaccharides that are components of glycosaminoglycans, which constitute a major part of the matrix of all connective tissues. The entry of GlcN into cells is stimulated by insulin and involves glucose-transporters (GLUTs), GLUT1 and GLUT2. GLUT1 has similar affinity for glucose and GlcN, while GLUT2 has about 20-fold higher affinity for GlcN than glucose [192]. GLUT1 and GLUT2 are expressed in a variety of tumors including breast invasive ductal carcinoma. The same GLUTs are not expressed in normal breast tissue [76]. Thus, the binding of GlcN could be an attractive therapeutic approach for GLUTs mediated delivery of anticancer drugs. Kumar et al. [112] modified poly(amidoamine), or PAMAM dendrimers, obtained by the interactive branching of $\beta$-alanine repeat units, with L-tryptophan and GlcN (PTN) for higher drug loading and to use GLUT transporters, respectively. DOX-loaded PTN demonstrated $\mathrm{pH}$-sensitive drug release with significant higher cytotoxicity against breast cancer cells than PAMAM (Fig. 4a) [112]. GLUT1 is overexpressed in breast cancer stem-like cell (CSC) subpopulation [211]. Yi et al. [211] modified glucose-installed poly(ethylene glycol)-block-poly(L-lysine) with lipoic acid at the $\omega$-end (Glu-PEG-PLL-LA). Glu-PEG-PLL-LA was associated with a single siRNA to form an unimer-polyion, which was used to decorate gold NPs through Au-S bonding. With respect to glucose-unconjugated control NPs, conjugated NPs exhibited higher cellular uptake of siRNA payloads in spheroid breast cancer cells (MBA-MB-231), especially in the CSC fraction, probably due to their higher GLUT1 expression level [211]. It was demonstrated that tumor cells compete with host cells for glutamine (Gln), which causes Gln to flux from normal tissues to the tumor [197]. Wang et al. [197] exploited Gln transporter SLC1A5 (solute carrier family 1 member 5 ) to target the polyglutamine-based NCs delivering therapeutic small interfering RNAs (siRNAs) into orthotopic lung tumor cells. Gamma-ray emitting 111In, which is extensively used for imaging, is also a source of short-range Auger electrons (AE). While exhibiting negligible effect outside cells, these AE become highly toxic near DNA within the cell nucleus. Therefore, these radionuclides can be used as a therapeutic anticancer agent if delivered precisely into the nuclei of tumor target cells [197]. A modular nanotransporter loading AE emitter 111In has also been developed by Rosenkranz et al. [165] to kill human bladder cancer cells overexpressing the EGFR [165].

\section{Enzymes}

The development of safe and effective stimuli responsive NCs is very important for tumor chemotherapy [214]. In particular, due to their high specificity to bind a certain substrate, molecules responsive to these specific stimuli are enzymes. Prodrug cancer therapy involves the selective activation of prodrug(s) in tumor tissues by exogenous enzyme(s). As a result, the activated prodrug could kill cancer cells. A peptide linker, mimicking the enzyme substrate conjugated between the drug and the polymeric carrier, is responsible for the drug release mechanism [186]. Jiang et al. [96] synthesized hyaluronidase enzyme-responsive targeted NPs for effective delivery of 5-FU in colon cancer. These NPs were threefold more effective than free drug and twofold more effective than 5-FU loaded mesoporous silica NPs [96].

\section{Antibodies}

Monoclonal antibodies are produced by a single clone of cells. They are engineered to recognize and bind to a single specific antigen as well as highly specific toward a circulat- 
ing protein or cells that have the corresponding antigenic structure on their surfaces. This high specificity renders antibodies suitable tools for delivering payloads to specific targets, for example, cytotoxic drugs to malignant cells or enzymes to specific cell types [160]. Lim et al. [121] described a self-assembled protein NC capable of delivering functional antibodies to the cytosol and generated intracellular therapeutics without systemic cytotoxicity. This NC has been made by combining a $\alpha$-helical peptide that is selfassembled into a hexameric coiled-coil bundle with an Fc-binding protein A fragment. Regardless of the $\mathrm{pH}$ and the antibody's originating species, the localization of multiple Fc-binding domains on the hexameric core allows to bind the antibody with a subnanomolar affinity. A cytosol-delivering capability of this NC was provided by using anti $\beta$-tubulin or antinuclear pore complex antibody as cargo. In addition, an endosomolytic component conjugated to the NC improved the cytosolic delivery effectiveness of the Hex NC. As a result, this modified Hex NC exhibited similar antibodybinding properties but delivered more antibodies to their cytosolic targets at a faster rate than other reported antibody carriers (Fig. 4c) [121].

\section{Hybrid DDSs}

The current trend of DDSs for cancer targeting and treatment is the synthesis of hybrid DDSs combining the properties of various biomaterials and NCs. Due to their enhanced effect on tumor therapeutics, these multifunctional NCs are widely used in therapy [199]. For example, Prusty et al. [156], synthesized lipid-mediated self-assembly of aptamers, and combined different biomaterials into a single nanoconstruct that targets the hepatocyte growth factor receptor (cMet). This $\mathrm{NC}$ consists of lipid derivatives of a cMet-binding aptamer and a separate lipid derivative of GC-rich DNA hairpin loaded with intercalated DOX. Furthermore, multiple $2^{\prime}, 6^{\prime}$-dimethylazobenzene moieties were incorporated into the DOX-binding polymer to trigger the release of the chemotherapeutics by photoisomerization. The resulting NC increased: (i) the serum nuclease resistance, (ii) the relative transfection into the cells mediated by endocytosis, and (iii) the selective photo-release of chemotherapeutics into the targeted cells [156]. Biodegradable core-shell lipid-polymer hybrid NPs can combine the advantages of lipid and polymeric NPs for controlled drug delivery [181]. Song et al. [181] synthetized lipid-polymer hybrid NPs conjugated with EGF co-delivering docetaxel and resveratrol for the treatment of advanced non-small cell lung cancer. In vitro and in vivo studies demonstrated that these NPs have significant synergistic effects, the best tumor inhibition ability, and the lowest systemic toxicity [181]. Zhang et al. [214] used bovine serum albumin and green tea polyphenol to synthe- size glutathione and enzyme (trypsin) responsive NCs [215, 216] for DOX delivery [214].

These new-generation DDSs still face multiple challenges. These hurdles include the accessibility/identification of suitable/novel receptors, transporters and enzymes, the lack of knowledge about differential expression between healthy and cancer cells, the selection of ligands (cell surface receptors and transporters), enzymes, immunological kinetics and/or click chemistry (coating molecules and payloads), the improvement of the entrapment efficiency/release of payloads, the drug degradation and the NC stability.

\section{Future Perspectives and Conclusions}

NC-based DDSs have recently gained attention in nanomedicine for the treatment of various diseases, and particularly cancer. Polymeric, lipid, and hybrid NCs are used to develop safe, biocompatible, and advanced DDSs loading payloads with different physicochemical properties and modulate the cargo release as a specific response to physical and chemical stimuli. Biomaterials used for the synthesis of NCs have different compositions and functional groups that can be simultaneously or not conjugated with peptides, antibodies, and small molecules to provide a selective active targeting. Payloads used for cancer drug delivery are natural and/or synthetic molecules that can affect, as well as NCs per se, the costs of anticancer therapy. Various strategies for targeting have been developed to increase the effectiveness of the therapy and improve patient compliance. Targeting approaches, biomaterials, and payloads as well as biopharmaceutical properties of DDSs affect anticancer therapy and impact the healthcare and patient quality of life. A similar effect was recently obtained for other pathologies, such as metabolic, cardiovascular, and infection diseases. The wide diffusion of selective molecules, providing specific targeting, novel and smart biomaterials as well as natural and synthetic payloads with potent therapeutic efficacy, allowed the synthesis of smart NCs and the development of modern DDSs. This scenario is slowly transforming lethal diseases, such as some cancers, into chronic diseases while developing the medicine for the future, focused on patient's quality of life and overall survival. NCs help nanotechnology and nanomedicines to treat tumor selectively due to the recent development of smart and novel DDSs using physical/ chemical stimuli and molecules generated from microenvironment of tumors. These factors are part of the immune systems of patients and are specific targets suitable for personalized medicines. Drug degradation, stability of NCs, and scale-up still remain a major challenge to be address. Although freeze-drying allows stabilizing NCs and preserving NPs as well as payloads from chemical and bio- 
logical degradation, the lyophilization process can partially inactivate targeting molecules and decrease their selectivity against specific receptors and transporters overexpressed on the surface of cancer cells and tissues. To date, a new frontier for personalized medicines and selective targeting of DDSs is the synthesis of lipid micelles conjugating targeting molecules that are postinserted in preformulated lyophilized NCs that are dissolved in sterile water or saline solution before patient injection. This strategy opens a new scenario in the targeting strategies and allows to preserve both biopharmaceutical properties of NCs either the therapeutic activity of payloads for long storage without affecting targeting properties. Furthermore, targeting molecules that are specific for their tumor markers can be cloned and collected from patients thus improving the efficacy of cancer therapy and decreasing the side effects and related failure. This approach may represent a new frontier for future experimental protocols and clinical studies.

Many studies currently need acute and chronic toxicities of DDSs used in anticancer therapy. The lack of toxicity data depends on missing data (meta-analysis) in this field due to the restricted access of patients to DDSs cure for cancer therapy which remains a second line of treatment for different carcinoma. In addition, several patients with cancer have a limited rate of survival and a low perspective on life for many aggressive carcinomas.

\section{Bibliographic Database Search (Carried Out on March 22, 2019 at 12:30 AM)}

The following query string was entered into the searchcommand-line of the database of the peer-reviewed literature Scopus (Elsevier):

TITLE-ABS-KEY ("nanocarrier" AND "drug delivery" AND “cancer") AND (LIMIT-TO (PUBSTAGE, "final”)) AND (LIMIT-TO (PUBYEAR, 2019) OR LIMIT-TO (PUBYEAR, 2018)) AND (LIMIT-TO (DOCTYPE, “ar")) AND (LIMIT-TO (SUBJAREA, "PHAR") OR LIMIT-TO (SUBJAREA, "BIOC") OR LIMIT-TO (SUBJAREA, "CHEM") OR LIMIT-TO (SUBJAREA, "MEDI") OR LIMIT-TO (SUBJAREA, "IMMU")) AND (LIMIT-TO (LANGUAGE, "English")).

The query string was structured to retrieve manuscripts that include in the title/abstract/keywords regarding NCs in the field of the application of DDSs for cancer applications.

\section{Boolean/Proximity Operators and Wildcard Characters}

The following Boolean/Proximity operators and Wildcard characters were adopted to define the query strings:

1. "' quotation marks. They allow one to find the terms between the quotation marks in the exact order they are specified (exact sentence), avoiding sentences with reversed terms.

2. () round brackets. They allow to find the composition of complex search expressions by defining the research priorities.

3. AND. It allows to find records in which they are present all the expressions simultaneously.

\section{General Database Settings}

The following general settings were adopted:

1. English language.

2. Search by topic (title, abstract, and keywords).

3. Timespan from 2018 to 2019.

4. Document type (articles and reviews).

5. Subject area (pharmacology, (bio)chemistry, medicine, and immunology).

Bibliographic searches by using other bibliographic databases (PubMed, Web Of Science) were also performed.

\section{Manuscript Selection}

The selection of manuscripts has been made manually by topic (title, abstract, and keywords), without considering their impact (number of citation or IF/SJR of the Journal). Among the papers (articles and reviews) retrieved in the last 2 years (more than 523 papers, without duplicates), only appropriate findings involving the subject areas of pharmacology, (bio)chemistry, medicine, and immunology were discussed. Older papers were included in the discussion just in case they were essential for the description of various topics. In cases where two or more papers discuss the same topic, the most recent, or the one containing more data, was considered. 
Acknowledgments The researches of Nadeem Muhammad Hafeez, and Dalila Iannotta are funded by the Italian Ministry of Instruction, University, and Research under the national project Programma Operativo Nazionale Ricerca e Innovazione (PON) 2014-2020 (CCI 2014IT16M2OP005) Fondo Sociale Europeo, Azione I.1, Dottorati Innovativi con Caratterizzazione Industriale.

\section{References}

1. Accomasso, L., Cristallini, C., \& Giachino, C. (2018). Risk assessment and risk minimization in nanomedicine: A need for predictive, alternative, and 3Rs strategies. Frontiers in Pharmacology, 9 , 228.

2. Aftab, S., Shah, A., Nadhman, A., Kurbanoglu, S., Aysıl Ozkan, S., Dionysiou, D. D., Shukla, S. S., \& Aminabhavi, T. M. (2018). Nanomedicine: An effective tool in cancer therapy. International Journal of Pharmaceutics, 540, 132-149.

3. Agrawal, S., Kelkar, M., De, A., et al. (2018). Newly emerging mesoporous strontium hydroxyapatite nanorods: Microwave synthesis and relevance as doxorubicin nanocarrier. Journal of Nanoparticle Research, 20, 230.

4. Ai, S., Zhen, S., Liu, Z., et al. (2018). An iRGD peptide conjugated heparin nanocarrier for gastric cancer therapy. RSC Advances, 8 , 30012-30020.

5. Al-Ahmady, Z. S., Hadjidemetriou, M., Gubbins, J., et al. (2018). Formation of protein corona in vivo affects drug release from temperature-sensitive liposomes. Journal of Controlled Release, 276, 157-167.

6. Ak, G., Yilmaz, H., Güneş, A., et al. (2018). In vitro and in vivo evaluation of folate receptor-targeted a novel magnetic drug delivery system for ovarian cancer therapy. Artificial Cells, Nanomedicine, and Biotechnology, 46(Suppl), 926-937.

7. Albanese, A., Tang, P. S., \& Chan, W. C. (2012). The effect of nanoparticle size, shape, and surface chemistry on biological systems. Annual Review of Biomedical Engineering, 14, 1-16.

8. Albano, J. M., De Morais Ribeiro, L. N., Couto, V. M., et al. (2019). Rational design of polymer-lipid nanoparticles for docetaxel delivery. Colloids and Surfaces B: Biointerfaces, 175, $56-64$.

9. Alsaleh, N. B., \& Brown, J. M. (2018). Immune responses to engineered nanomaterials: Current understanding and challenges. Current Opinion in Toxicology, 10, 8-14.

10. Amoli-Diva, M., Sadighi-Bonabi, R., Pourghazi, K., et al. (2018). Tunable surface plasmon resonance-based remote actuation of bimetallic core-shell nanoparticle-coated stimuli responsive polymer for switchable chemo-photothermal synergistic cancer therapy. Journal of Pharmaceutical Sciences, 107, 2618-2627.

11. Argenziano, M., Lombardi, C., Ferrara, B., et al. (2018). Glutathione/pH-responsive nanosponges enhance strigolactone delivery to prostate cancer cells. Oncotarget, 9, 35813-35829.

12. Arvizo, R. R., Miranda, O. R., Moyano, D. F., et al. (2011). Modulating pharmacokinetics, tumor uptake and biodistribution by engineered nanoparticles. PLoS One, 6, e24374.

13. Attia, M. F., Anton, N., Wallyn, J., et al. (2019). An overview of active and passive targeting strategies to improve the nanocarriers efficiency to tumour sites. The Journal of Pharmacy and Pharmacology, 71, 1185-1198.

14. Bahuguna, S., Kumar, M., Sharma, G., et al. (2018). Fullerenolbased intracellular delivery of methotrexate: A water-soluble nanoconjugate for enhanced cytotoxicity and improved pharmacokinetics. AAPS PharmSciTech, 19, 1084-1092.

15. Banala, V. T., Sharma, S., Barnwal, P., et al. (2018). Synchronized ratiometric codelivery of metformin and topotecan through engineered nanocarrier facilitates in vivo synergistic precision levels at tumor site. Advanced Healthcare Materials, 7, 1800300.
16. Bansal, S., Singh, J., Kumari, U., et al. (2019). Development of biosurfactant-based graphene quantum dot conjugate as a novel and fluorescent theranostic tool for cancer. International Journal of Nanomedicine, 14, 809.

17. Bazak, R., Houri, M., Achy, S. E., et al. (2014). Passive targeting of nanoparticles to cancer: A comprehensive review of the literature. Molecular and Clinical Oncology, 2, 904-908.

18. BBCResearch. (2015). Nanotechnology in medical applications: the global market. http://www.bccresearch.com/market-research/ healthcare/nanotechnology-medical-applications-markethlc069c.html. Accessed 9 Nov 2019.

19. Behr, J. (1997). The proton sponge: A trick to enter cells the viruses did not exploit. Chimia, 51, 34-36.

20. Beija, M., Salvayre, R., Lauth-De Viguerie, N., et al. (2012). Colloidal systems for drug delivery: From design to therapy. Trends in Biotechnology, 30, 485-496.

21. Bergers, G., \& Benjamin, L. E. (2003). Tumorigenesis and the angiogenic switch. Nature Reviews. Cancer, 3, 401-410.

22. Bikram, M., Gobin, A. M., Whitmire, R. E., et al. (2007). Temperature-sensitive hydrogels with $\mathrm{SiO} 2-\mathrm{Au}$ nanoshells for controlled drug delivery. Journal of Controlled Release, 123, 219-227.

23. Bikram, M., \& West, J. L. (2008). Thermo-responsive systems for controlled drug delivery. Expert Opinion on Drug Delivery, 5, 1077-1091.

24. Bilobrov, V., Sokolova, V., Prylutska, S., et al. (2019). A novel nanoconjugate of landomycin A with $\mathrm{C} 60$ fullerene for cancer targeted therapy: In vitro studies. Cellular and Molecular Bioengineering, 12, 41-51.

25. Blanco, E., Shen, H., Ferrari, M., et al. (2015). Principles of nanoparticle design for overcoming biological barriers to drug delivery. Nature Biotechnology, 33, 941-951.

26. Buhleier, E., Wehner, W., \& Vögtle, F. (1978). "Cascade"- and "nonskid-chain-like" syntheses of molecular cavity topologies. Synthesis, 1978, 155-158.

27. Braun, G. B., Sugahara, K. N., Yu, O. M., et al. (2016). Urokinasecontrolled tumor penetrating peptide. Journal of Controlled Release, 232, 188-195.

28. Bugno, J., Hsu, H.-J., Pearson, R. M., et al. (2016). Size and surface charge of engineered poly(amidoamine) dendrimers modulate tumor accumulation and penetration: A model study using multicellular tumor spheroids. Molecular Pharmaceutics, 13, 2155-2163.

29. Candido, N. M., De Melo, M. T., Franchi, L. P., et al. (2018). Combining photodynamic therapy and chemotherapy: Improving breast cancer treatment with nanotechnology. Journal of Biomedical Nanotechnology, 14, 994-1008.

30. Cao, D., Liang, L., Xu, Y., et al. (2018b). Tumor associated macrophages and angiogenesis dual-recognizable nanoparticles for enhanced cancer chemotherapy. Nanomedicine, 14, 651-659.

31. Cao, J., Wang, C., Guo, L., et al. (2018a). Co-administration of a charge-conversional dendrimer enhances antitumor efficacy of conventional chemotherapy. European Journal of Pharmaceutics and Biopharmaceutics, 127, 371-377.

32. Cellante, L., Costa, R., Monaco, I., et al. (2018). One-step esterification of nanocellulose in a Brønsted acid ionic liquid for delivery to glioblastoma cancer cells. New Journal of Chemistry, 42, 5237-5242.

33. Charbgoo, F., Nikkhah, M., \& Behmanesh, M. (2018). Size of single-wall carbon nanotube affects the folate receptor-mediated cancer cell targeting. Biotechnology and Applied Biochemistry, 65, 328-337.

34. Chaumet, A., Wright, G. D., Seet, S. H., et al. (2015). Nuclear envelope-associated endosomes deliver surface proteins to the nucleus. Nature Communications, 6, 8218.

35. Chen, H. H., Lu, I. L., Liu, T. I., et al. (2019). Indocyanine green/ doxorubicin-encapsulated functionalized nanoparticles for effec- 
tive combination therapy against human MDR breast cancer. Colloids and Surfaces. B, Biointerfaces, 177, 294-305.

36. Chen, T., Gong, T., Zhao, T., et al. (2017). A comparison study between lycobetaine-loaded nanoemulsion and liposome using nRGD as therapeutic adjuvant for lung cancer therapy. European Journal of Pharmaceutical Sciences, 111, 293-302.

37. Chen, T., Gong, T., Zhao, T., et al. (2018b). A comparison study between lycobetaine-loaded nanoemulsion and liposome using nRGD as therapeutic adjuvant for lung cancer therapy. European Journal of Pharmaceutical Sciences, 111, 293-302.

38. Chen, W. L., Yang, S. D., Li, F., et al. (2018a). Programmed pH/ reduction-responsive nanoparticles for efficient delivery of antitumor agents in vivo. Acta Biomaterialia, 81, 219-230.

39. Chitgupi, U., Shao, S., Carter, K. A., et al. (2018). Multicolor liposome mixtures for selective and selectable cargo release. Nano Letters, 18, 1331-1336.

40. Daga, M., Ullio, C., Argenziano, M., et al. (2016). GSH-targeted nanosponges increase doxorubicin-induced toxicity "in vitro" and "in vivo" in cancer cells with high antioxidant defenses. Free Radical Biology \& Medicine, 97, 24-37.

41. Danson, S., Ferry, D., Alakhov, V., et al. (2004). Phase I dose escalation and pharmacokinetic study of pluronic polymer-bound doxorubicin (SP1049C) in patients with advanced cancer. British Journal of Cancer, 90, 2085-2091.

42. Dassie, J. P., Hernandez, L. I., Thomas, G. S., et al. (2014). Targeted inhibition of prostate cancer metastases with an RNA aptamer to prostate-specific membrane antigen. Molecular Therapy, 22, 1910-1922.

43. Dautry-Varsat, A. (1986). Receptor-mediated endocytosis: The intracellular journey of transferrin and its receptor. Biochimie, 68, 375-381.

44. Dramou, P., Fizir, M., Taleb, A., et al. (2018). Folic acid-conjugated chitosan oligosaccharide-magnetic halloysite nanotubes as a delivery system for camptothecin. Carbohydrate Polymers, 197, $117-127$.

45. Dresselhaus, M. S., Dresselhaus, G., \& Eklund, P. C. (1996). Science of fullerenes and carbon nanotubes: Their properties and applications. Cambridge: Academic Press.

46. Du, M., Ouyang, Y., Meng, F., et al. (2019). Polymer-lipid hybrid nanoparticles: A novel drug delivery system for enhancing the activity of psoralen against breast cancer. International Journal of Pharmaceutics, 561, 274-282.

47. Du, X. L., Chan, W., Giordano, S., et al. (2005). Variation in modes of chemotherapy administration for breast carcinoma and association with hospitalization for chemotherapy-related toxicity. Cancer, 104, 913-924.

48. Duan, Q., Ma, Y., Che, M., et al. (2019). Fluorescent carbon dots as carriers for intracellular doxorubicin delivery and track. Journal of Drug Delivery Science and Technology, 49, 527-533.

49. Dufes, C., Uchegbu, I. F., \& Schätzlein, A. G. (2005). Dendrimers in gene delivery. Advanced Drug Delivery Reviews, 57, 2177-2202.

50. Dutta Chowdhury, A., Ganganboina, A. B., Tsai, Y. C., et al. (2018). Multifunctional GQDs-Concanavalin A@Fe3O4 nanocomposites for cancer cells detection and targeted drug delivery. Analytica Chimica Acta, 1027, 109-120.

51. Elhissi, A., Ahmed, W., Dhanak, V., et al. (2012). Carbon nanotubes in cancer therapy and drug delivery. In K. Subramani \& W. Ahmed (Eds.), Emerging nanotechnologies in dentistry (1st ed., pp. 347-363). Waltham: William Andrew Publishing (Elsevier).

52. Erdoğar, N., Esendağlı, G., Nielsen, T. T., et al. (2018). Therapeutic efficacy of folate receptor-targeted amphiphilic cyclodextrin nanoparticles as a novel vehicle for paclitaxel delivery in breast cancer. Journal of Drug Targeting, 26, 66-74.
53. Etheridge, M. L., Campbell, S. A., Erdman, A. G., et al. (2013). The big picture on nanomedicine: The state of investigational and approved nanomedicine products. Nanomedicine, 9, 1-14.

54. Fadeel, D. A., Al-Toukhy, G. M., Elsharif, A. M., et al. (2018). Improved photodynamic efficacy of thiophenyl sulfonated zinc phthalocyanine loaded in lipid nano-carriers for hepatocellular carcinoma cancer cells. Photodiagnosis and Photodynamic Therapy, 23, 25-31.

55. Fan, J., Wang, S., Sun, W., et al. (2018). Anticancer drug delivery systems based on inorganic nanocarriers with fluorescent tracers. AICHE Journal, 64, 835-859.

56. Fang, J., Liu, Y., Chen, Y., et al. (2018b). Graphene quantum dots-gated hollow mesoporous carbon nanoplatform for targeting drug delivery and synergistic chemo-photothermal therapy. International Journal of Nanomedicine, 13, 5991-6007.

57. Fang, J., Nakamura, H., \& Maeda, H. (2011). The EPR effect: Unique features of tumor blood vessels for drug delivery, factors involved, and limitations and augmentation of the effect. Advanced Drug Delivery Reviews, 63, 136-151.

58. Fang, S., Hou, Y., Ling, L., et al. (2018a). Dimeric camptothecin derived phospholipid assembled liposomes with high drug loading for cancer therapy. Colloids and Surfaces B: Biointerfaces, 166, 235-244.

59. Farjadian, F., Ghasemi, A., Gohari, O., Roointan, A., Karimi, M., \& Hamblin, M. R. (2019). Nanopharmaceuticals and nanomedicines currently on the market: Challenges and opportunities. Nanomedicine (London, England), 14, 93-126.

60. Feng, W., Han, X., Wang, R., et al. (2019). Nanocatalystsaugmented and photo-thermal-enhanced tumor-specific sequential nanocatalytic therapy in both NIR-I and NIR-II biowindows. Advanced Materials, 31, e1805919.

61. Fenske, D. B., \& Cullis, P. R. (2008). Liposomal nanomedicines. Expert Opinion on Drug Delivery, 5, 25-44.

62. Fernandes, C., Suares, D., \& Yergeri, M. C. (2018b). Tumor microenvironment targeted nanotherapy. Frontiers in Pharmacology, 9, 1230.

63. Fernandes, R. S., Silva, J. O., Mussi, S. V., et al. (2018a). Nanostructured lipid carrier co-loaded with doxorubicin and docosahexaenoic acid as a theranostic agent: Evaluation of biodistribution and antitumor activity in experimental model. Molecular Imaging and Biology, 20, 437-447.

64. Ferrara, N., Hillan, K. J., \& Novotny, W. (2005). Bevacizumab (Avastin), a humanized anti-VEGF monoclonal antibody for cancer therapy. Biochemical and Biophysical Research Communications, 333, 328-335.

65. Flühmann, B., Ntai, I., Borchard, G., et al. (2019). Nanomedicines: The magic bullets reaching their target? European Journal of Pharmaceutical Sciences, 128, 73-80.

66. Frigerio, B., Bizzoni, C., Jansen, G., et al. (2019). Folate receptors and transporters: Biological role and diagnostic/therapeutic targets in cancer and other diseases. Journal of Experimental \& Clinical Cancer Research, 38, 125.

67. Fuhrmann, K., Gauthier, M. A., \& Leroux, J.-C. (2014). Targeting of injectable drug nanocrystals. Molecular Pharmaceutics, 11, $1762-1771$.

68. Fuhrmann, K., Połomska, A., Aeberli, C., et al. (2013). Modular design of redox-responsive stabilizers for nanocrystals. ACS Nano, 7, 8243-8250.

69. Fuhrmann, K., Schulz, J. D., Gauthier, M. A., et al. (2012). PEG nanocages as non-sheddable stabilizers for drug nanocrystals. ACS Nano, 6, 1667-1676.

70. Ganta, S., Paxton, J. W., Baguley, B. C., et al. (2009). Formulation and pharmacokinetic evaluation of an asulacrine nanocrystalline suspension for intravenous delivery. International Journal of Pharmaceutics, 367, 179-186. 
71. Gao, J., Yu, B., Li, C., et al. (2019). Ultrasound triggered phasechange nanodroplets for doxorubicin prodrug delivery and ultrasound diagnosis: An in vitro study. Colloids and Surfaces. B, Biointerfaces, 174, 416-425.

72. Gao, Z., Lukyanov, A. N., Singhal, A., et al. (2002). Diacyllipidpolymer micelles as nanocarriers for poorly soluble anticancer drugs. Nano Letters, 2, 979-982.

73. Garay, R. P., El-Gewely, R., Armstrong, J. K., et al. (2012). Antibodies against polyethylene glycol in healthy subjects and in patients treated with PEG-conjugated agents. Expert Opinion on Drug Delivery, 9, 1319-1323.

74. Geng, S., Wu, L., Cui, H., et al. (2018). Synthesis of lipid-black phosphorus quantum dot bilayer vesicles for near-infraredcontrolled drug release. Chemical Communications (Cambridge), 54, 6060-6063.

75. Ghorbani, M., Mahmoodzadeh, F., Nezhad-Mokhtari, P., et al. (2018). A novel polymeric micelle-decorated Fe3O4/Au coreshell nanoparticle for $\mathrm{pH}$ and reduction-responsive intracellular co-delivery of doxorubicin and 6-mercaptopurine. New Journal of Chemistry, 42, 18038-18049.

76. Godoy, A., Ulloa, V., Rodríguez, F., et al. (2006). Differential subcellular distribution of glucose transporters GLUT1-6 and GLUT9 in human cancer: Ultrastructural localization of GLUT1 and GLUT5 in breast tumor tissues. Journal of Cellular Physiology, 207, 614-662.

77. Gonzales, J., Kossatz, S., Roberts, S., et al. (2018). Nanoemulsion-based delivery of fluorescent PARP inhibitors in mouse models of small cell lung cancer. Bioconjugate Chemistry, 29, 3776-3782.

78. Granger, D. N., \& Senchenkova, E. (2010). Chapter 7: Leukocyteendothelial cell adhesion. In D. N. Granger \& E. Senchenkova (Eds.), Inflammation and the microcirculation (pp. 29-40). San Rafael: Morgan \& Claypool Life Sciences.

79. Gref, R., Lück, M., Quellec, P., et al. (2000). 'Stealth' corona-core nanoparticles surface modified by polyethylene glycol (PEG): Influences of the corona (PEG chain length and surface density) and of the core composition on phagocytic uptake and plasma protein adsorption. Colloids and Surfaces. B, Biointerfaces, 18, 301-313.

80. Griffin, L. C., Tidmarsh, G. F., Bock, L. C., et al. (1993). In vivo anticoagulant properties of a novel nucleotide-based thrombin inhibitor and demonstration of regional anticoagulation in extracorporeal circuits. Blood, 81, 3271-3276.

81. Guaita-Esteruelas, S., Gumà, J., Masana, L., et al. (2018). The peritumoural adipose tissue microenvironment and cancer. The roles of fatty acid binding protein 4 and fatty acid binding protein. Molecular and Cellular Endocrinology, 462(Pt B), 107-118.

82. Gui, W., Zhang, J., Chen, X., et al. (2018). N-Doped graphene quantum dot@ mesoporous silica nanoparticles modified with hyaluronic acid for fluorescent imaging of tumor cells and drug delivery. Microchimica Acta, 185, 66.

83. Guo, Y., Zhao, S., Qiu, H., et al. (2018). Shape of nanoparticles as a design parameter to improve docetaxel antitumor efficacy. Bioconjugate Chemistry, 29, 1302-1311.

84. Gupta, N., Jangid, A. K., Singh, M., et al. (2018). Designing twodimensional nanosheets for improving drug delivery to Fucosereceptor-overexpressing cancer cells. ChemMedChem, 13, 2644-2652.

85. Hadilou, N., Khoshgenab, A. N., Amoli-Diva, M., et al. (2018). Remote trice light, temperature, and $\mathrm{pH}$-actuation of switchable magneto-plasmonic nanocarriers for combinational photothermal and controlled/targeted chemotherapies. Journal of Pharmaceutical Sciences, 107, 3123-3133.

86. Hadjidemetriou, M., McAdam, S., Garner, G., et al. (2019). The human in vivo biomolecule Corona onto PEGylated liposomes:
A proof-of-concept clinical study. Advanced Materials, 31, e1803335.

87. Hanafy, N. A. N., El-Kemary, M., \& Leporatti, S. (2018). Micelles structure development as a strategy to improve smart cancer therapy. Cancers (Basel). https://doi.org/10.3390/ cancers 10070238.

88. He, C. B., Hu, Y. P., Yin, L. C., et al. (2010). Effects of particle size and surface charge on cellular uptake and biodistribution of polymeric nanoparticles. Biomaterials, 31, 3657-3666.

89. Hao, G., Xu, Z. P., \& Li, L. (2018). Manipulating extracellular tumour $\mathrm{pH}$ : An effective target for cancer therapy. RSC Advances, 8, 22182-22192.

90. Hollis, C. P., Weiss, H. L., Leggas, M., et al. (2013). Biodistribution and bioimaging studies of hybrid paclitaxel nanocrystals: Lessons learned of the EPR effect and image-guided drug delivery. Journal of Controlled Release, 172, 12-21.

91. Huang, R., Li, J., Kebebe, D., et al. (2018). Cell penetrating peptides functionalized gambogic acid-nanostructured lipid carrier for cancer treatment. Drug Delivery, 25, 757-765.

92. Huang, Y. F., Shangguan, D. H., Liu, H. P., et al. (2009). Molecular assembly of an aptamer-drug conjugate for targeted drug delivery to tumor cells. Chembiochem, 10, 862-868.

93. Iijima, S. (1991). Helical microtubules of graphitic carbon. Nature, 354, 56-58.

94. Irby, D., Du, C., \& Li, F. (2017). Lipid-drug conjugate for enhancing drug delivery. Molecular Pharmaceutics, 14, 1325-1338.

95. Jahan, S. T., Sadat, S. M. A., Walliser, M., \& Haddadi, A. (2017). Targeted therapeutic nanoparticles: An immense promise to fight against cancer. Journal of Drug Delivery, 2017, 9090325.

96. Jiang, H., Shi, X., Yu, X., et al. (2018). Hyaluronidase enzymeresponsive targeted nanoparticles for effective delivery of 5-fluorouracil in colon cancer. Pharmaceutical Research, 35, 73.

97. Jin, R., Liu, Z., Bai, Y., et al. (2018). Multiple-responsive mesoporous silica nanoparticles for highly accurate drugs delivery to tumor cells. ACS Omega, 3, 4306-4315.

98. Kang, S., Kim, E. H., Hwang, J. E., Shin, J. H., Jeong, Y. S., Yim, S. Y., Joo, E. W., Eun, Y. G., Lee, D. J., Sohn, B. H., Lee, S. H., Lim, B., \& Lee, J. S. (2019). Prognostic significance of high metabolic activity in breast cancer: PET signature in breast cancer. Biochemical and Biophysical Research Communications, 511, 185-191.

99. Karandish, F., Froberg, J., Borowicz, P., et al. (2018). Peptidetargeted, stimuli-responsive polymersomes for delivering a cancer stemness inhibitor to cancer stem cell microtumors. Colloids and Surfaces. B, Biointerfaces, 163, 225-235.

100. Kateb, B., Yamamoto, V., Yu, C., et al. (2009). Infrared thermal imaging: A review of the literature and case report. NeuroImage, 47, T154-T162.

101. Kaur, H., Bruno, J. G., Kumar, A., \& Sharma, T. K. (2018). Aptamers in the therapeutics and diagnostics pipelines. Theranostics, 8, 4016-4032.

102. Kayani, Z., Firuzi, O., \& Bordbar, A. K. (2018). Doughnut-shaped bovine serum albumin nanoparticles loaded with doxorubicin for overcoming multidrug-resistant in cancer cells. International Journal of Biological Macromolecules, 107, 1835-1843.

103. Key, J., Palange, A. L., Gentile, F., et al. (2015). Soft discoidal polymeric nanoconstructs resist macrophage uptake and enhance vascular targeting in tumors. ACS Nano, 9, 11628-11641.

104. Khajavinia, A., Varshosaz, J., \& Dehkordi, A. J. (2012). Targeting etoposide to acute myelogenous leukaemia cells using nanostructured lipid carriers coated with transferrin. Nanotechnology, 23, 405101.

105. Khatri, S., Lohani, P., \& Gandhi, S. (2013). Nanoemulsions in cancer therapy. Indo Global Journal of Pharmaceutical Sciences, $3,124-133$. 
106. Khoee, M. H., Khoee, S., \& Lotfi, M. (2019). Synthesis of titanium dioxide nanotubes with liposomal covers for carrying and extended release of 5-FU as anticancer drug in the treatment of HeLa cells. Analytical Biochemistry, 572, 16-24.

107. Kim, S., Shi, Y., Kim, J. Y., et al. (2010). Overcoming the barriers in micellar drug delivery: Loading efficiency, in vivo stability, and micelle-cell interaction. Expert Opinion on Drug Delivery, 7, 49-62.

108. Kopeckova, K., Eckschlager, T., Sirc, J., et al. (2019). Nanodrugs used in cancer therapy. Biomedical Papers of the Medical Faculty of the University Palacky, Olomouc, Czech Republic. https://doi. org/10.5507/bp.2019.010.

109. Koshkaryev, A., Sawant, R., Deshpande, M., et al. (2013). Immunoconjugates and long circulating systems: Origins, current state of the art and future directions. Advanced Drug Delivery Reviews, 65, 24-35.

110. Kostarelos, K., Lacerda, L., Partidos, C., et al. (2005). Carbon nanotube-mediated delivery of peptides and genes to cells: Translating nanobiotechnology to therapeutics. Journal of Drug Delivery Science and Technology, 15, 41-47.

111. Kumar, A., \& Dhawan, A. (Eds.). (2019). Nanoparticle-protein corona: Biophysics to biology. Cambridge: Royal Society of Chemistry.

112. Kumar, S. S. D., Mahesh, A., Antoniraj, M. G., et al. (2018). Cellular imaging and folate receptor targeting delivery of gum kondagogu capped gold nanoparticles in cancer cells. International Journal of Biological Macromolecules, 109, 220-230.

113. Kutova, O. M., Guryev, E. L., Sokolova, E. A., et al. (2019). Targeted delivery to tumors: Multidirectional strategies to improve treatment efficiency. Cancers (Basel), 11(1), 68. Pii:E68.

114. Lakhin, A. V., Tarantul, V. Z., Gening, L. V., et al. (2013). Aptamers: Problems, solutions and prospects. Acta Naturae, 5, 34-43.

115. Le Trinh, T., Zhu, G. Z., Xiao, X. L., et al. (2015). A synthetic aptamer-drug adduct for targeted liver cancer therapy. PLoS One, 10, $\mathrm{e} 0136673$.

116. Lee, K. S., Chung, H. C., Im, S. A., et al. (2008). Multicenter phase II trial of Genexol-PM, a Cremophor-free, polymeric micelle formulation of paclitaxel, in patients with metastatic breast cancer. Breast Cancer Research and Treatment, 108, 241-250.

117. Li, S. D., \& Huang, L. (2006). Surface-modified LPD nanoparticles for tumor targeting. Annals of the New York Academy of Sciences, 1082, 1-8.

118. Li, W., Li, Z., Wei, L., et al. (2018). Evaluation of paclitaxel nanocrystals in vitro and in vivo. Drug Research, 68, 205-212.

119. Liao, W., Zhang, L., Zhong, Y., et al. (2018). Fabrication of ultrasmall WS2 quantum dots-coated periodic mesoporous organosilica nanoparticles for intracellular drug delivery and synergistic chemo-photothermal therapy. Oncotargets and Therapy, 11, 1949.

120. Licciardi, M., Paolino, D., Celia, C., et al. (2010). Folate-targeted supramolecular vesicular aggregates based on polyas-partylhydrazide copolymers for the selective delivery of antitumoral drugs. Biomaterials, 31, 7340-7354.

121. Lim, S. I., Lukianov, C. I., \& Champion, J. A. (2017). Selfassembled protein nanocarrier for intracellular delivery of antibody. Journal of Controlled Release, 249, 1-10.

122. Lin, F., Wen, D., Wang, X., et al. (2019). Dual responsive micelles capable of modulating miRNA-34a to combat taxane resistance in prostate cancer. Biomaterials, 192, 95-108.

123. Liu, D., Yang, F., Xiong, F., et al. (2016). The smart drug delivery system and its clinical potential. Theranostics, 6, 1306-1323.

124. Liu, J., Huang, Y., Kumar, A., Tan, A., Jin, S., Mozhi, A., \& Liang, X. J. (2014). pH-sensitive nano-systems for drug delivery in cancer therapy. Biotechnology Advances, 32, 693-710.

125. Liu, Z., Wang, F., \& Chen, X. (2008). Integrin alpha(v)beta(3)-targeted cancer therapy. Drug Development Research, 69, 329-339.
126. Lopez-Toledano, M. A., Saxena, V., Legassie, J. D., et al. (2019). Advanced lipid technologies ${ }^{\circledR}$ (ALT®): A proven formulation platform to enhance the bioavailability of lipophilic compounds. Journal of Drug Delivery, 2019, 1957360.

127. Loppinet, B., \& Monteux, C. (2016). Dynamics of surfactants and polymers at liquid interfaces. In P. Lang \& Y. Liu (Eds.), Soft matter at aqueous interfaces (pp. 137-157). Heidelberg: Springer.

128. Maeda, H., \& Khatami, M. (2018). Analyses of repeated failures in cancer therapy for solid tumors: Poor tumor-selective drug delivery, low therapeutic efficacy and unsustainable costs. Clinical and Translational Medicine, 7, 11.

129. Maeda, H., Sawa, T., \& Konno, T. (2001). Mechanism of tumortargeted delivery of macromolecular drugs, including the EPR effect in solid tumor and clinical over-view of the prototype polymeric drug SMANCS. Journal of Controlled Release, 74, 47-46.

130. Mahmoodzadeh, F., Jannat, B., \& Ghorbani, M. (2019). Chitosan-based nanomicelle as a novel platform for targeted delivery of methotrexate. International Journal of Biological Macromolecules, 126, 517-524.

131. Mao, J., Ran, D., Xie, C., et al. (2017). EGFR/EGFRvIII dualtargeting peptide-mediated drug delivery for enhanced glioma therapy. ACS Applied Materials \& Interfaces, 9, 24462-24475.

132. Martinez, F. O., \& Gordon, S. (2014). The M1 and M2 paradigm of macrophage activation: Time for reassessment. F1000Prime Reports, 6, 13.

133. Mathews, A. S., Ha, C. S., Cho, W. J., et al. (2006). Drug delivery system based on covalently bonded poly[N-isopropylacrylamideco-2-hydroxyethylacrylate]-based nanoparticle networks. Drug Delivery, 13, 245-251.

134. Matsumura, Y., Hamaguchi, T., Ura, T., et al. (2004). Phase I clinical trial and pharmacokinetic evaluation of NK911, a micelle-encapsulated doxorubicin. British Journal of Cancer, 91, $1775-1781$.

135. Mauricio, M. D., Guerra-Ojeda, S., Marchio, P., et al. (2018). Nanoparticles in medicine: A focus on vascular oxidative stress. Oxidative Medicine and Cellular Longevity, 2018, 6231482.

136. McClements, D. J. (2015). Nanoscale nutrient delivery systems for food applications: Improving bioactive dispersibility, stability, and bioavailability. Journal of Food Science, 80, N1602-N1611.

137. Michanetzis, G. P., Markoutsa, E., Mourtas, S., et al. (2019). Hemocompatibility of amyloid and/or brain targeted liposomes. Future Medicinal Chemistry. https://doi.org/10.4155/ fmc-2018-0236.

138. Migotto, A., Carvalho, V. F., Salata, G. C., et al. (2018). Multifunctional nanoemulsions for intraductal delivery as a new platform for local treatment of breast cancer. Drug Delivery, 25, 654-667.

139. Mu, L., Chrastina, A., Levchenko, T., et al. (2005). Micelles from poly (ethylene glycol)-phosphatidyl ethanolamine conjugates (peg-Pe) as pharmaceutical nanocarriers for poorly soluble drug camptothecin. Journal of Biomedical Nanotechnology, 1, $190-195$.

140. Müller, R. H., Gohla, S., \& Keck, C. M. (2011). State of the art of nanocrystals-special features, production, nanotoxicology aspects and intracellular delivery. European Journal of Pharmaceutics and Biopharmaceutics, 78, 1-9.

141. Nagamitsu, A., Greish, K., \& Maeda, H. (2009). Elevating blood pressure as a strategy to increase tumor-targeted delivery of macromolecular drug SMANCS: Cases of advanced solid tumors. Japanese Journal of Clinical Oncology, 39, 756-766.

142. Narmani, A., Mohammadnejad, J., \& Yavari, K. (2019). Synthesis and evaluation of polyethylene glycol-and folic acid-conjugated polyamidoamine G4 dendrimer as nanocarrier. Journal of Drug Delivery Science and Technology, 50, 278-286.

143. Nielsen, U. B., Kirpotin, D. B., Pickering, E. M., et al. (2002). Therapeutic efficacy of anti-ErbB2 immunoliposomes tar- 
geted by a phage antibody selected for cellular endocytosis. Biochimica et Biophysica Acta (BBA)-Molecular Cell Research, 1591, 109-118.

144. Normanno, N., De Luca, A., Bianco, C., Strizzi, L., Mancino, M., Maiello, M. R., Carotenuto, A., De Feo, G., Caponigro, F., \& Salomon, D. S. (2006). Epidermal growth factor receptor (EGFR) signaling in cancer. Gene, 366, 2-16.

145. Owens, D. E., 3rd, \& Peppas, N. A. (2006). Opsonization, biodistribution, and pharmacokinetics of polymeric nanoparticles. International Journal of Pharmaceutics, 307, 93-102.

146. Palange, A. L., Palomba, R., Rizzuti, I. F., et al. (2017). Deformable discoidal polymeric nanoconstructs for the precise delivery of therapeutic and imaging agents. Molecular Therapy, $25,1514-1521$.

147. Paolino, D., Licciardi, M., Celia, C., et al. (2012). Folate-targeted supramolecular vesicular aggregates as a new frontier for effective anticancer treatment in in vivo model. European Journal of Pharmaceutics and Biopharmaceutics, 82, 94-102.

148. Peetla, C., Stine, A., \& Labhasetwar, V. (2009). Biophysical interactions with model lipid membranes: Applications in drug discovery and drug delivery. Molecular Pharmaceutics, 6, 1264-1276.

149. Pelaz, B., Alexiou, C., Alvarez-Puebla, R. A., et al. (2017). Diverse applications of nanomedicine. ACS Nano, 11, 2313-2381.

150. Peng, E., Choo, E. S., Tan, C. S., et al. (2013). Multifunctional PEGylated nanoclusters for biomedical applications. Nanoscale, 5, 5994-6005.

151. Pokharkar, V. B., Jolly, M. R., \& Kumbhar, D. D. (2015). Engineering of a hybrid polymer-lipid nanocarrier for the nasal delivery of tenofovir disoproxil fumarate: Physicochemical, molecular, microstructural, and stability evaluation. European Journal of Pharmaceutical Sciences, 71, 99-111.

152. Polomska, A., Gauthier, M. A., \& Leroux, J. C. (2017). In vitro and in vivo evaluation of PEGylated layer-by-layer polyelectrolytecoated paclitaxel nanocrystals. Small. https://doi.org/10.1002/ smll.201602066.

153. Poudel, B. K., Soe, Z. C., Ruttala, H. B., et al. (2018). In situ fabrication of mesoporous silica-coated silver-gold hollow nanoshell for remotely controllable chemo-photothermal therapy via phase-change molecule as gatekeepers. International Journal of Pharmaceutics, 548, 92-103.

154. Pourjavadi, A., Asgari, S., Hosseini, S. H., et al. (2018). Codelivery of hydrophobic and hydrophilic drugs by graphene-decorated magnetic dendrimers. Langmuir, 34, 15304-15318.

155. Prabhakar, U., Maeda, H., Jain, R. K., et al. (2013). Challenges and key considerations of the enhanced permeability and retention effect for nanomedicine drug delivery in oncology. Cancer Research, 73, 2412-2417.

156. Prusty, D. K., Adam, V., Zadegan, R. M., et al. (2018). Supramolecular aptamer nano-constructs for receptor-mediated targeting and light-triggered release of chemotherapeutics into cancer cells. Nature Communications, 9, 535.

157. Prylutska, S., Panchuk, R., Gołuński, G., et al. (2017a). C 60 fullerene enhances cisplatin anticancer activity and overcomes tumor cell drug resistance. Nano Research, 10, 652-671.

158. Prylutska, S., Politenkova, S., Afanasieva, K., et al. (2017b). A nanocomplex of C60 fullerene with cisplatin: Design, characterization and toxicity. Beilstein Journal of Nanotechnology, 8 , 1494-1501.

159. Qi, S. S., Sun, J. H., Yu, H. H., et al. (2017). Co-delivery nanoparticles of anti-cancer drugs for improving chemotherapy efficacy. Drug Delivery, 24, 1909-1926.

160. Ranade, V. V. (1989). Drug delivery systems-2. Site-specific drug delivery utilizing monoclonal antibodies. Journal of Clinical Pharmacology, 29, 873-884.

161. Raza, A., Rasheed, T., Nabeel, F., et al. (2019). Endogenous and exogenous stimuli-responsive drug delivery systems for programmed site-specific release. Molecules, 24, 1117.
162. Rezaei, S. J. T., Norouzi, K., Hesami, A., et al. (2018). Au (III) complexes loaded $\mathrm{pH}$-responsive magnetic nanogels for cancer therapy. Applied Organometallic Chemistry, 32, e4303.

163. Riley, R. S., June, C. H., Langer, R., et al. (2019). Delivery technologies for cancer immunotherapy. Nature, 18, 175-196.

164. Roncato, F., Rruga, F., Porcù, E., et al. (2018). Improvement and extension of anti-EGFR targeting in breast cancer therapy by integration with the Avidin-nucleic-acid-nano-assemblies. Nature Communications, 9, 4070.

165. Rosenkranz, A. A., Slastnikova, T. A., Karmakova, T. A., et al. (2018). Antitumor activity of Auger electron emitter 111In delivered by modular nanotransporter for treatment of bladder cancer with EGFR overexpression. Frontiers in Pharmacology, 9, 1331.

166. Rossmanna, C., \& Haemmerich, D. (2014). Review of temperature dependence of thermal properties, dielectric properties, and perfusion of biological tissues at hyperthermic and ablation temperatures. Critical Reviews in Biomedical Engineering, 42, 467-492.

167. Sarker, D. K. (2005). Engineering of nanoemulsions for drug delivery. Current Drug Delivery, 2, 297-310.

168. Sawa-Wejksza, K., \& Kandefer-Szerszeń, M. (2018). Tumorassociated macrophages as target for antitumor therapy. Archivum Immunologiae et Therapiae Experimentalis (Warsz), 66, 97-111.

169. Schatz, A. A., Prejsnar, K. W., McCanney, J., et al. (2019). Policy strategies for the "new normal" in healthcare to ensure access to high-quality cancer care. Journal of the National Comprehensive Cancer Network, 17, 105-109.

170. Schramm, L. L., Stasiuk, E. N., \& Marangoni, D. G. (2003). 2 surfactants and their applications. Annual Reports on the Progress of Chemistry, Section C: Physical Chemistry, 99, 3-48.

171. Shah, P., Bhalodia, D., \& Shelat, P. (2010). Nanoemulsion: A pharmaceutical review. Systematic Reviews in Pharmacy, 1, 24-32.

172. Sharma, P., Zujovic, Z. D., Bowmaker, G. A., et al. (2011). Evaluation of a crystalline nanosuspension: Polymorphism, process induced transformation and in vivo studies. International Journal of Pharmaceutics, 408, 138-151.

173. Shen, Y., Li, X., Dong, D., Zhang, B., Xue, Y., \& Shang, P. (2018). Transferrin receptor 1 in cancer: A new sight for cancer therapy. American Journal of Cancer Research, 8, 916-931.

174. Shi, J., Kantoff, P. W., Wooster, R., et al. (2017). Cancer nanomedicine: Progress, challenges and opportunities. Nature Reviews. Cancer, 17, 20.

175. Shi, Y., Van Der Meel, R., Theek, B., et al. (2015). Complete regression of xenograft tumors upon targeted delivery of paclitaxel via П-П stacking stabilized polymeric micelles. ACS Nano, 9, 3740-3752.

176. Shigdar, S., Macdonald, J., O'Connor, M., et al. (2013). Aptamers as theranostic agents: Modifications, serum stability and functionalisation. Sensors (Basel), 13, 13624-13637.

177. Shim, W. S., Yoo, J. S., Bae, Y. H., et al. (2005). Novel injectable $\mathrm{pH}$ and temperature sensitive block copolymer hydrogel. Biomacromolecules, 6, 2930-2934.

178. Shutava, T. G., Pattekari, P. P., Arapov, K. A., et al. (2012). Architectural layer-by-layer assembly of drug nanocapsules with PEGylated polyelectrolytes. Soft Matter, 8, 9418-9427.

179. Siegler, E. L., Kim, Y. J., \& Wang, P. (2016). Nanomedicine targeting the tumor microenvironment: Therapeutic strategies to inhibit angiogenesis, remodel matrix, and modulate immune responses. Journal of Cellular Immunotherapy, 2, 69-78.

180. Song, X., Wan, Z., Chen, T., et al. (2016). Development of a multitarget peptide for potentiating chemotherapy by modulating tumor microenvironment. Biomaterials, 108, 44-56.

181. Song, Z., Shi, Y., Han, Q., et al. (2018). Endothelial growth factor receptor-targeted and reactive oxygen species-responsive lung cancer therapy by docetaxel and resveratrol encapsulated lipidpolymer hybrid nanoparticles. Biomedicine \& Pharmacotherapy, $105,18-26$. 
182. Stearns, L. J., Narang, S., Albright, R. E., Jr., et al. (2019). Assessment of health care utilization and cost of targeted drug delivery and conventional medical management vs conventional medical management alone for patients with cancer-related pain. JAMA Network Open, 2, e191549.

183. Su, Y., Liu, M., Liang, K., et al. (2018). Evaluating the accelerated blood clearance phenomenon of PEGylated nanoemulsions in rats by intraperitoneal administration. AAPS PharmSciTech, 19, 3210-3218.

184. Sutradhar, K. B., \& Amin, M. D. L. (2014). Nanotechnology in cancer drug delivery and selective targeting. ISRN Nanotechnology, 2014, ID939378.

185. Tabatabaei, S. N., Derbali, R. M., Yang, C., et al. (2019). Co-delivery of miR-181a and melphalan by lipid nanoparticles for treatment of seeded retinoblastoma. Journal of Controlled Release, 298, 177-185.

186. Taghizadeh, B., Taranejoo, S., Monemian, S. A., et al. (2015). Classification of stimuli-responsive polymers as anticancer drug delivery systems. Drug Delivery, 22, 145-155.

187. Teesalu, T., Sugahara, K. N., Kotamraju, V. R., et al. (2009). $\mathrm{C}$-end rule peptides mediate neuropilin-1-dependent cell, vascular, and tissue penetration. Proceedings of the National Academy of Sciences of the United States of America, 106, 16157-16162.

188. Teesalu, T., Sugahara, K. N., \& Ruoslahti, E. (2013). Tumorpenetrating peptides. Frontiers in Oncology, 3, 216.

189. Tesarova, B., Charousova, M., Dostalova, S., et al. (2019). Folic acid-mediated re-shuttling of ferritin receptor specificity towards a selective delivery of highly cytotoxic nickel(II) coordination compounds. International Journal of Biological Macromolecules, 126, 1099-1111.

190. Tombak, A. (2019). Introductory chapter: Erythrocytes - Basis of life. In A. Tombak (Ed.), Erythrocytes (pp. 1-4). London: IntechOpen.

191. Torrisi, M. R., Lotti, L. V., Belleudi, F., et al. (1999). Eps15 is recruited to the plasma membrane upon epidermal growth factor receptor activation and localizes to components of the endocytic pathway during receptor internalization. Molecular Biology of the Cell, 10, 417-434.

192. Uldry, M., Ibberson, M., Hosokawa, M., et al. (2002). GLUT2 is a high affinity glucosamine transporter. FEBS Letters, 524, 199-203.

193. Vermeulen, L. M. P., De Smedt, S. C., Remaut, K., et al. (2018). The proton sponge hypothesis: Fable or fact? European Journal of Pharmaceutics and Biopharmaceutics, 129, 184-190.

194. Villaverde, G., \& Baeza, A. (2019). Targeting strategies for improving the efficacy of nanomedicine in oncology. Beilstein Journal of Nanotechnology, 10, 168-181.

195. Wadhawan, A., Chatterjee, M., \& Singh, G. (2019). Present scenario of bioconjugates in cancer therapy: A review. International Journal of Molecular Sciences, 20, E5243.

196. Wang, B.-H., Fan, J.-L., Wang, X.-W., et al. (2015). A nile blue based infrared fluorescent probe: Imaging tumors that over-express cyclooxygenase-2. Chemical Communications, 51, $792-795$.

197. Wang, C., Wu, J., Wang, Z., et al. (2018). Glutamine addiction activates polyglutamine-based nanocarriers delivering therapeutic siRNAs to orthotopic lung tumor mediated by glutamine transporter SLC1A5. Biomaterials, 183, 77-92.

198. Wang, F., Ye, X., Wu, Y., et al. (2019b). Time interval of two injections and first-dose dependent of accelerated blood clearance phenomenon induced by PEGylated liposomal gambogenic acid: The contribution of PEG-specific IgM. Journal of Pharmaceutical Sciences-Us, 108, 641-651.

199. Wang, Y., Wang, Z., Qian, Y., et al. (2019a). Synergetic estrogen receptor-targeting liposome nanocarriers with anti-phagocytic properties for enhanced tumor theranostics. Journal of Materials Chemistry B, 7, 1056-1063.

200. Weissleder, R. (2001). A clearer vision for in vivo imaging. Nature Biotechnology, 19, 316-317.

201. WHO (2019) Cancer. https://www.who.int/news-room/factsheets/detail/cancer. Accessed 8 Nov 2019.

202. Wilhelm, S., Tavares, A. J., Dai, Q., et al. (2016). Analysis of nanoparticle delivery to tumours. Nature Reviews Materials, 1, 16014.

203. Wu, X. Y. (2016). Strategies for optimizing polymer-lipid hybrid nanoparticle-mediated drug delivery. Expert Opinion on Drug Delivery, 13, 609-612.

204. Xiao, K., Li, Y., Luo, J., et al. (2011). The effect of surface charge on in vivo biodistribution of PEG-oligocholic acid based micellar nanoparticles. Biomaterials, 32, 3435-3446.

205. Xuan, M., Shao, J., Zhao, J., et al. (2018). Magnetic mesoporous silica nanoparticles cloaked by red blood cell membranes: Applications in cancer therapy. Angewandte Chemie (International Ed. in English), 57, 6049-6053.

206. Xu, R., Zhang, G., Mai, J., et al. (2016). An injectable nanoparticle generator enhances delivery of cancer therapeutics. Nature Biotechnology, 34, 414-418.

207. Yang, J., Lu, W., Xiao, J., et al. (2018a). A positron emission tomography image-guidable unimolecular micelle nanoplatform for cancer theranostic applications. Acta Biomaterialia, 79, 306-316.

208. Yang, X.-Z., Dou, S., Wang, Y.-C., et al. (2012). Single-step assembly of cationic lipid-polymer hybrid nanoparticles for systemic delivery of siRNA. ACS Nano, 6, 4955-4965.

209. Yang, Y., Chen, Q., Li, S., et al. (2018c). iRGD-mediated and enzyme-induced precise targeting and retention of gold nanoparticles for the enhanced imaging and treatment of breast cancer. Journal of Biomedical Nanotechnology, 14, 1396-1408.

210. Yang, Y., Zhao, Y., Lan, J., et al. (2018b). Reduction-sensitive CD44 receptor-targeted hyaluronic acid derivative micelles for doxorubicin delivery. International Journal of Nanomedicine, 13, 4361-4378.

211. Yi, Y., Kim, H. J., Zheng, M., et al. (2019). Glucose-linked sub50-nm unimer polyion complex-assembled gold nanoparticles for targeted siRNA delivery to glucose transporter 1-overexpressing breast cancer stem-like cells. Journal of Controlled Release, 295, 268-277.

212. Yu, M., Han, S., Kou, Z., et al. (2018). Lipid nanoparticle-based co-delivery of epirubicin and BCL-2 siRNA for enhanced intracellular drug release and reversing multidrug resistance. Artificial Cells, Nanomedicine, and Biotechnology, 46, 323-332.

213. Yu, Z., Pestell, T. G., Lisanti, M. P., et al. (2012). Cancer stem cells. The International Journal of Biochemistry \& Cell Biology, 44, 2144-2151.

214. Zhang, J., Ren, X., Tian, X., et al. (2019c). GSH and enzyme responsive nanospheres based on self-assembly of green tea polyphenols and BSA used for target cancer chemotherapy. Colloids and Surfaces. B, Biointerfaces, 173, 654-661.

215. Zhang, L., Hao, P. Y., Yang, D. J., et al. (2019b). Designing nanoparticles with improved tumor penetration: Surface properties from the molecular architecture viewpoint. Journal of Materials Chemistry B, 7, 953-964.

216. Zhang, R. X., Cai, P., Zhang, T., et al. (2016). Polymer-lipid hybrid nanoparticles synchronize pharmacokinetics of co-encapsulated doxorubicin-mitomycin $\mathrm{C}$ and enable their spatiotemporal codelivery and local bioavailability in breast tumor. Nanomedicine, $12,1279-1290$.

217. Zhang, W., Wen, Y., He, D.-X., et al. (2019a). Near-infrared AIEgens as transformers to enhance tumor treatment efficacy with controllable self-assembled redox-responsive carrier-free nanodrug. Biomaterials, 193, 12-21. 
218. Zhao, R., Zheng, G., Fan, L., et al. (2018a). Carrier-free nanodrug by co-assembly of chemotherapeutic agent and photosensitizer for cancer imaging and chemo-photo combination therapy. Acta Biomaterialia, 70, 197-210.

219. Zhao, X., Tian, K., Zhou, T., et al. (2018b). PEGylated multiwalled carbon nanotubes as versatile vector for tumor-specific intracellular triggered release with enhanced anti-cancer effi- ciency: Optimization of length and PEGylation degree. Colloids and Surfaces. B, Biointerfaces, 168, 43-49.

220. Zhao, Z., Ukidve, A., Krishnan, V., et al. (2019). Effect of physicochemical and surface properties on in vivo fate of drug nanocarriers. Advanced Drug Delivery Reviews. https://doi.org/10.1016/j. addr.2019.01.002. 


\title{
Homing Peptides for Cancer Therapy
}

\author{
Prakash Lingasamy and Tambet Teesalu
}

\section{Abstract}

Tumor-homing peptides are widely used for improving tumor selectivity of anticancer drugs and imaging agents. The goal is to increase tumor uptake and reduce accumulation at nontarget sites. Here, we describe current approaches for tumor-homing peptide identification and validation, and provide comprehensive overview of classes of tumor-homing peptides undergoing preclinical and clinical development. We focus on unique mechanistic features and applications of a recently discovered class of tumor-homing peptides, tumor-penetrating C-end Rule (CendR) peptides, that can be used for tissue penetrative targeting of extravascular tumor tissue. Finally, we discuss unanswered questions and future directions in the field of development of peptide-guided smart drugs and imaging agents.

\section{Keywords}

Tumor-homing peptide - Tumor-penetrating peptide . Nanoparticle $\cdot$ Nanomedicine $\cdot$ C-end Rule . Neuropilin-1 · Angiogenic integrins · gC1qR/p32 . Extracellular matrix $\cdot$ Multistep targeting $\cdot$ In vivo peptide phage display $\cdot \mathrm{T} 7$ phage $\cdot$ Affinity chromatography

P. Lingasamy

Laboratory of Cancer Biology, Institute of Biomedicine and Translational Medicine, University of Tartu, Tartu, Estonia

T. Teesalu $(\bowtie)$

Laboratory of Cancer Biology, Institute of Biomedicine and

Translational Medicine, University of Tartu, Tartu, Estonia

Center for Nanomedicine and Department of Cell, Molecular and Developmental Biology, University of California,

Santa Barbara, CA, USA

Cancer Research Center, Sanford Burnham Prebys Medical

Discovery Institute, La Jolla, CA, USA

e-mail: Tambet.Teesalu@ut.ee

\section{Introduction}

According to the World Health Organization (WHO), cancerrelated deaths are expected to pass the mortality of cardiovascular disease by 2030 [30]. Application of conventional therapies (surgery, radiotherapy, and chemotherapy) for the treatment of solid tumors has resulted in most cases only in incremental increases in the survival of patients with advanced disease [15]. The major chemotherapeutic drugs are low molecular weight chemical agents used to directly or indirectly inhibit the proliferation of rapidly growing cells. In general, the chemotherapy agents kill cancer cells by interacting with the DNA/RNA synthesis function of the cells and the cell cycle function. Chemotherapeutic drugs are categorized into different classes based on their mechanism of action [77]: (1) antimetabolites that mimic the building blocks of RNA and DNA; (2) alkaloids and alkylating agents that directly damage DNA; (3) antitumor antibiotics that interfere with the enzymes involved in DNA replication; (4) topoisomerase inhibitors that inhibit either topoisomerase I or II enzymes involved in unwinding DNA during replication and transcription; (5) mitotic inhibitors that disrupt microtubules causing inhibition of mitosis, or cell division; and (6) steroid hormones involved in estrogen and progesterone and relieve the side effects from other drugs.

Most chemotherapeutic drugs are administered via a systemic route, with the exposure of both healthy and cancerous cells in the body. Unfortunately, a major problem in systemic therapy is that the chemo drugs are nonspecific and attack not only malignant cells but also healthy proliferating cells. Typically only a small fraction of administered drugs reaches its target site(s) and the chemotherapeutics administered over an extended period cause severe toxic side effects, such as damage to the gastrointestinal mucosa and hematopoietic cells, mouth and throat sores, weight loss and cachexia, organ damage, nausea, vomiting, diarrhea, anemia, infections, fatigue, and destruction of the immune system [36]. 
Administering chemo drugs over extended periods of time leads to the loss of the ability to kill malignant cells and to emergence of resistance. To minimize the side effects, subtherapeutic doses of anticancer drugs are frequently used, the strategy that results in a selection of cancerous cells resistant to the drug, and, ultimately, in more aggressive disease [10]. Monitoring tumor response - clinical symptoms and tumor size - with imaging techniques such as computed tomography (CT), magnetic resonance imaging (MRI), positron emission tomography (PET), and radiography, is critical for effective cancer treatment strategies. Tumor size dynamics (shrinkage vs. progression) is widely used as a criterion for a response; however, some drugs that stabilize disease without an objective response may slow tumor growth sufficiently to improve patient survival [202]. Cancer treatment schemes are based on classification of tumor at diagnosis and clinical symptoms. However, genetic profiles of tumors with the same diagnosis, and hence, sensitivity to therapeutic interventions, can vary, leading to application of nonefficacious treatment strategies and poor prognosis. It has been estimated that the rate of application of inefficient treatment strategies varies from $29.8 \%$ to $42.5 \%$, and reaches in some cases even $100 \%$ [202].

Cancerous tissue is heterogeneous, with striking regional differences in the tumor structure and physiology that translate into variability in the uptake and distribution of anticancer drugs within tumor tissue. Approved anticancer drugs, even cytotoxic/static compounds with low molecular weight, show inefficient extravasation and penetration of malignant tissue and, therefore, limited therapeutic efficacy [106, 200]. Application of tumor and patient-specific markers for selective delivery of chemotherapeutics to malignant lesions may restrict the damage to healthy tissues and increase therapeutic efficacy $[45,114]$. Targeting ligands can also be used for noninvasive patient stratification and better monitoring of treatment response. Therefore, next-generation smart cancer therapeutics should include built-in homing mechanisms that result in their preferential accumulation in malignant tissues [202].

\subsection{Next-Generation Cancer Therapies}

A recent progress in preclinical and clinical cancer research has led to the development of novel transformative intervention strategies such as immunotherapies, personalized molecular therapies, nanomedicine, and affinity cancer targeting.

\subsubsection{Immunotherapy}

As part of its normal function, the immune system detects and destroys abnormal cells and most likely prevents or curbs the growth of many cancers. Malignant cells have evolved to avoid destruction by the immune system by a number of mechanisms, including: introducing genetic changes that make them less visible to the immune system, upregulating proteins on their surface that turn off immune cells, and modulating the tumor microenvironment to interfere with how the immune system responds to the cancer cells $[32,69,75]$. The aim of cancer immunotherapies is to restore the ability of the immune system to better recognize and fight against cancer. The first generation of cancer immunotherapies are immune checkpoint inhibitors (anti-CTLA-4 and anti-PD-1/PD-L1) that target natural immune homeostasis pathways to drive antitumor immune responses [32]. In a subset of patients, these checkpoint inhibitors have proven successful in curing metastatic diseases such as nonsmall cell lung cancer and melanoma. Additional immuno-based strategies include T-cell transfer therapy to boost the natural ability of $\mathrm{T}$ cells to fight cancer; treatment vaccines, which boost immune system's response to cancer cells, and immune system modulators, which enhance the body's immune response against cancer [35, 92].

The key challenge in cancer immunotherapies is to increase the responder patient population. There is a need for new antitumor immune-activating agents, which are currently in an advanced stages of development in preclinical/ clinical studies [22].

\subsubsection{Personalized Molecular Therapies}

The development of every cancer is driven by a unique set of abnormalities in its genetic makeup that are present only in the tumor and not in normal tissues. While different patients may be diagnosed with what appears to be the same type of cancer, the genetic makeup, underlying mechanisms, and efficient therapeutic disease management strategies vary from patient to patient. Personalized molecular therapies are designed to attack specific mutations and other cancer-related changes in cells to suppress malignant cell proliferation and induce apoptosis by targeting specific molecules that play a critical role in cellular activities. One strategy uses humanized monoclonal antibodies (hMoAb) such as Rituximab (target: CD20), cetuximab (EGFR), and Bevacizumab (VEGF) [11, 23]. The second approach is using tyrosine kinase inhibitors such as cabozantinib (VEGFR2 inhibitor) and vandetanib (a VEGFR and EGFR inhibitor) gefitinib, and imatinib. Personalized molecular therapies require molecular profiling of patients to characterize potential targets for choosing the right drug combinations. However, the off-target toxicities and development of resistance remain significant concerns. The ongoing development of complex genomic and molecular companion diagnostic assays could help to stratify patients and focus on cohorts most likely to respond to drugs. 


\subsubsection{Nanomedicine}

Nanomedicine drugs are seen as the "the magic bullets" that could improve cancer therapy and ameliorate the treatment of malignant disease by being more effective in reaching the target sites, have increased therapeutic efficacy, and cause less adverse side effects. The key revolutionary aspect of nanomedicine lies in the versatility and multifunctionality of the nanocarriers. Multitude of nanoformulations of different compositions, sizes, shapes, and surface properties, have been designed for therapeutic, diagnostic, and theranostic applications. Nanocarriers can accommodate therapeutic and diagnostic payloads of different classes, and can be simultaneously loaded with drugs and imaging agents for theranostic applications - application of a single nanosystems for both detection/diagnosis and therapy of malignant disease [64]. Nanocarrier loading can dramatically extend the drug circulation time and improve the biodistribution for better tolerability and an improved therapeutic outcome. Various classes of NPs are approved for clinical use including liposomes, PEGylated liposomes, albumin NPs, and polymeric micelles. Many more NPs are in clinical and preclinical trials [106]. The first marketed nanodrug Doxil (PEGylated liposomal doxorubicin) was approved by the FDA in 1995 for the treatment of Kaposi's sarcoma [20,71]. Doxil and other approved nanodrugs are based on existing drugs that have been nanoformulated to improve their biodistribution or pharmacodynamic properties. All clinically approved nanoparticles are nontargeted passive drug delivery vehicles [28, 199]. Accumulation of such nanodrugs in solid tumors is believed to be due to an enhanced permeability and retention (EPR) effect, caused by the abnormal architecture of tumor blood vessels, lack of lymphatic drainage, defective endothelial cells with wide fenestrations, and inadequate pericyte coverage [120, $121,123,153]$. For nanomedicine to hold its promise of revolutionizing disease prevention, diagnosis, and treatment, the in vivo delivery and biodistribution of nanoparticles must be improved further by strategies such as active targeting.

\subsection{Active Cancer Targeting with Affinity Ligands}

Malignant cells and cells in tumor stroma express surface molecules that distinguish them from healthy quiescent cells in adult organisms. Targeting of systemically accessible parts of this heterogeneity with affinity ligands (e.g., peptides and antibodies) is also referred to as active (synaphic) targeting [169]. The intended outcome of the synaphic targeting is to achieve what is seen following topical application: high local accumulation with little or no sys- temic exposure. In contrast to EPR-based passive targeting of tumors that relies on leaky vessels and poor lymphatic drainage that result in the extravasation and accumulation of drugs in the tumor tissue [122], active targeting can be used to improve accumulation of therapeutic payloads in target tissue and their uptake in target cells, leading to increased therapeutic efficacy and reduced off-target side effects $[46,165]$. Affinity ligands to tumor vascular signature primarily target specific expression patterns in activated vascular cells (vascular and lymphatic endothelial cells and pericytes), many of which are secondary to the process of angiogenesis - sprouting of new blood vessels from existing vessels. Examples are cell surface angiogenic integrins, growth factor receptors, extracellular proteases, and extracellular matrix proteins. Importantly, by increasing the local drug concentration, active targeting also helps to lower the risk of developing drug resistance [13]. In the last three decades, genomics, proteomics and molecular profiling of cancers, and agnostic techniques such as in vivo peptide phage display, have resulted in the discovery of a number of cancer-specific receptors that can serve as targets for systemic circulating probes. The knowledge facilitated the development of affinity ligands of different classes such as Abs and their fragments, peptides, aptamers, and small molecules [114, 160, 171, 207]. An important challenge related to the ligand-based affinity targeting of vascular heterogeneity in malignant tissues relates to the limitation due to the number of accessible receptors in the tumor tissue $[81,131,166]$. One way to increase the capacity of affinity-based targeting systems is to develop multitargeted vascular targeting ligands and/or ligands that are capable of actively entering tumor parenchyma $[48,115,159]$.

A large number of biological ligands, including antibodies, polysaccharides, nucleic acids, peptides, and small molecules, have been studied as agents to facilitate active targeting of malignant disease [210]. Polypeptide-based targeting ligands, including homing peptides, protein domains, and antibodies, have advantages over other classes of targeting ligands in that they can be systematically developed using various biological selection and expression systems. Some major issues of protein ligands include immunogenicity, low stability, and difficulty for site-specific conjugation on nanoparticles [57, 187]. Antibody-drug conjugates (ADCs) are complexes of monoclonal antibodies (mAb) conjugated to drugs or radionuclide therapeutic payloads through chemical linkers. Three FDA-approved ADCs are available for cancer treatment: brentuximab vedotin (target: CD30), trastuzumab emtansine (target: HER2 receptor), and inotuzumab ozogamicin (target: CD22) and many more are in clinical trials [1]. Antibodies are attractive targeting ligands due to high specificity and versatility; however, 
antibody-based drugs have inherent limitations, such as immunogenicity, and high cost of production on a large scale $[23,111,171]$. Also, antibodies as other proteins of a similar size cannot efficiently extravasate and reach beyond $50 \mu \mathrm{m}$ in the tumor parenchyma $[66,176]$.

\subsubsection{Tumor-Homing Peptides}

Tumor-homing peptides have typically size $<20$ amino acids and are derived from natural domains of receptorbinding proteins, or have been identified by combinatorial approaches such as ribosomal and phage display. The key advantage of peptides over antibodies as affinity targeting ligands is that due to their small size they possess superior tissue and cell penetration ability [167, 193]. In addition, peptides tend to interact with conserved and functionally important binding pockets on the surface of target molecules, and thus are often biologically active. Importantly and of translational relevance, the small size of peptides renders them relatively nonimmunogenic. The recent advances in peptide synthesis technology allow for fully automated, facile synthesis and manufacturing scale-up of the production in a cost-effective manner - an important aspect considering clinical development and translation, especially considering the cost of large-scale manufacturing of antibodies. Homing peptides can be engineered to involve a range of nonnatural modifications to improve their target binding and/or stability, and to introduce functional groups for site-specific conjugation to proteins, nanocarriers, cytotoxic drugs, radionuclides, and toxins [15, 58, 104, 190].

On downside, peptides generally have lower target binding affinity and stability in biological fluids than antibodies. To compensate for the low affinity and to increase the avidity of target binding, multiple peptides can be used simultaneously. Proteolytic processing may be mechanistically important for triggering interaction of the peptide with its target, for example, activation of the cell and tumor penetration function of neuropilin-1 binding C-end Rule (CendR) peptides requires cleavage of the peptide by a tumor-derived protease to expose the C-terminal arginine residue $[9,167$, 192, 193]. In addition, the in vivo half-life of peptides can be modulated by conjugating to albumin-binding elements or polyethylene glycol coupling, substitution of $\mathrm{L}$ amino acids with $\mathrm{D}$-amino acids, blocking $\mathrm{N}$ - and $\mathrm{C}$-termini, introduction of lactam bridges, and cyclization [113, 146, 203].

In preclinical studies, peptide-based synaphic targeting efforts have led to an impressive improvement in the biodistribution of payloads and their therapeutic efficacy. A big challenge is to translate this success into clinical studies and FDA-approved therapeutic strategies and peptide-targeted anticancer payloads and imaging agents $[15,50,117,167$, 193, 194].

\section{Vascular Heterogeneity and Homing Peptide Discovery}

\subsection{Vascular Zip Codes}

The growth of tumors beyond microscopic lesions depends critically on their ability to ensure the supply of oxygen and nutrients through neovessels generated by angiogenesis and lymphangiogenesis $[68,168]$. In the absence of the angiogenic switch to trigger neovascularization, tumor growth is limited to less than $2 \mathrm{~mm}^{3}$ [133]. The angiogenic tumor blood vessels are morphologically, molecularly, and functionally different from healthy blood vessels. Characteristic microanatomical features of tumor vascular trees include irregular and torturous blood vessels with variable intravascular distances and irregular branching patterns, and the lack of an efficient lymphatic drainage system [44, 130]. Molecularly, the angiogenic signature of tumor neovessels involves upregulation of proangiogenic factors and markers that are not expressed or are expressed at a much lower level in healthy vessels, including angiogenic signaling molecules, adhesion receptors, extracellular matrix components, and enzymes that remodel the extracellular matrix. Examples are members of the angiogenic vascular endothelial growth factor (VEGF) family, basic fibroblast growth factor (bFGF), transforming growth factor (TGF)- $\alpha$ and $-\beta$, platelet-derived endothelial growth factor, granulocyte colony-stimulating factor, placental growth factor, epidermal growth factor, interleukin- 8 , hepatocyte growth factor, $\alpha v \beta 3$ and $\alpha v \beta 5$ integrins, oncofetal splice variants of fibronectin and Tenascin-C, matrix remodeling proteases - metalloproteinases, and urokinase-type plasminogen activator [158]. As discussed below, in activated cells in tumors some intracellular proteins such as nucleolin and $\mathrm{P} 32 / \mathrm{qC} 1 \mathrm{qR}$ are aberrantly expressed at the cell surface of the tumor and are accessible to the circulating affinity probes $[41,76,137,173,182,216]$. The lymphatic endothelial cells are also specialized, as they express markers that are not present in the lymphatics of healthy tissues, or in tumor blood vessels [109]. The term vascular ZIP code refers to this unique vasculo/lymphatic signature that can be applied for systemic synaphic (affinitybased) targeting of diagnostic and therapeutic cargo [164, 194]. Interestingly, the vascular signatures show overlap across a palette of different diseases: cancer, atherosclerosis, inflammation, thrombosis, sepsis, vascular leak syndrome, and tissue regeneration $[17,73,135,188]$. The likely reason is that all these conditions involve angiogenic/inflammatory components. At the same time, a subset of tumor ZIP codes and the corresponding homing peptides shows exquisite target selectivity and can, for example, differentiate between premalignant lesions and malignant cancer of the same tumor type and location $[12,165]$. An important aspect of 
systemic targeting of the vascular component of tumors is that, unlike mutation-prone tumor cells, nonmalignant cells of the tumor vasculature are genetically stable and less likely to develop drug resistance or mutate the peptide binding sites on receptors [138].

\subsection{Peptide Phage Display}

Bacteriophages (or phages) are viruses composed of a nucleocapsid that encapsulates the genetic material (DNA or RNA genome) that infects and replicates within the bacteria, but are harmless to humans [2, 21, 83, 112, 195]. The bacteriophage genome can be modified to encode foreign peptide sequences as fusions with the coat protein. George Smith and his Ph.D. student, Stephen F. Parmley were the first to insert foreign DNA fragments into filamentous phage gene encoding $\mathrm{p} 3$ protein - one of the minor coat proteins that decorates in five copies the emerging tip of the filamentous phage, to express p3-fusion peptides on the phage surface [183]. Greg Winter subsequently created first phage libraries for humanized antibody discovery, alternative to the hybridoma technology [43]. To contruct peptide display phage libraries, genes encoding phage coat protein are modified to insert a stretch of DNA encoding random peptide sequences at a diversity of about one billion variants per library, close to the total number of theoretical permutations of a random 7-amino acid sequence (1.28E9). Phage display is widely used for peptide and antibody discovery based on different bacteriophage vectors such as T7, T4, M13, and f1. The importance of the phage display technology was acknowledged in 2018 by awarding the Nobel Prize in Chemistry to George P. Smith and Sir Gregory P. Winter "for their contribution in the phage display of peptides and antibodies."

In the context of systemic affinity targeting, in vivo phage display has become an established agnostic tool for the identification of homing peptides and mapping sytemically accessible molecular heterogeneity in normal organs and diseased tissues [143]. This technology has enabled assessment of the extent of the molecular specialization in the vasculature and has contributed to identification of a number of new markers expressed in the tumor neovasculature [143, 165]. For in vivo selection, a peptide phage library is injected into the systemic circulation of animals, typically intravenously, followed by perfusion to remove the background phage, removal of target organs, amplification of the bound phage, and next round of selection (Fig. 1). Unlike in vitro and cellfree selection, in vivo peptide phage selections have an inbuilt mechanism against selecting the promiscous panspecific peptides due to their depletion in nontarget vascular beds [194]. For in vivo peptide display, our group prefers to use the lytic T7 bacteriophage system that, compared to lyso- genic filamentous phage-based display systems, has a size and aspect ratio more similar to those of clinically developed nanoparticles (in the case of $\mathrm{T} 7$ the nucleocapsid diameter is $\sim 55-60 \mathrm{~nm}$, whereas in the case of filamentous phage dimensions are $\sim 6 \mathrm{~nm} \times 900 \mathrm{~nm}$ ), is less restrictive for the amino acid sequence of displayed peptides, exhibits increased stability, and has a lower mutation rate. In the T7-Select system of Novagen, the peptide-encoding DNA is inserted at the C-terminus of the major coat protein $10 \mathrm{~A}$ or $10 \mathrm{~B}$ gene, displaying density of foreign peptides maximum up to 415 identical peptides/phage [50, 167, 194]. High-copy display results in high avidity binding, even in the case of lowaffinity peptide ligands, and reduced off-rates. Using phage display, tumor-homing peptides can be discovered using two different strategies: either using cell-free biopanning on purified target receptor (top-down approach), or in vivo selection with no a priori knowledge of the receptors (bottom down, or agnostic screening approach). A representative selection of tumor-targeting peptide identification and application data are stored in TumorHoPe [94] and THPdb [197] databases.

\subsubsection{In Vitro Biopanning for Homing Peptide Detection}

For top-down selection, the target for affinity delivery is chosen based on its absence in healthy tissues, upregulation in malignancy(ies) of interest, and systemic accessibility in the tumor tissue. The target recombinant protein is expressed, purified, immobilized, and used for peptide phage biopanning. The selection rounds are repeated until enrichment is achieved (usually in 3-6 rounds), followed by sequencing of peptide-encoding genomic DNA using Sanger or nextgeneration sequencing. The candidate peptide-phages are then back-cloned in the phage genomic DNA (to exclude contribution of mutations elsewhere in the phage capsid to binding) and validated for their target interaction (binding, specificity), and stability. A variation of the theme is to perform different rounds of biopanning on different target proteins to develop multitargeted peptides, as we have recently done for tumor-associated isoforms of extracellular matrix proteins (tenascin C and fibronectin) [115]. In some cases, target receptors fail to maintain the structural integrity during recombinant protein expression and engineered cells overexpressing target molecules can be used for biopanning. In the case of difficult targets, for example, members of highly conserved protein families, the specificity of the peptides can be improved by addition of negative selection steps. A related problem is that cell-free and in vitro selections fail to eliminate promiscuous peptides that nonselectively "stick" to nontarget components and thus lack intended selectivity. One possible way to address this problem is to combine in vitro biopanning with the in vivo selection described in the next section to deplete promiscuous peptides in nontarget organs. 


\section{In vivo phage display}

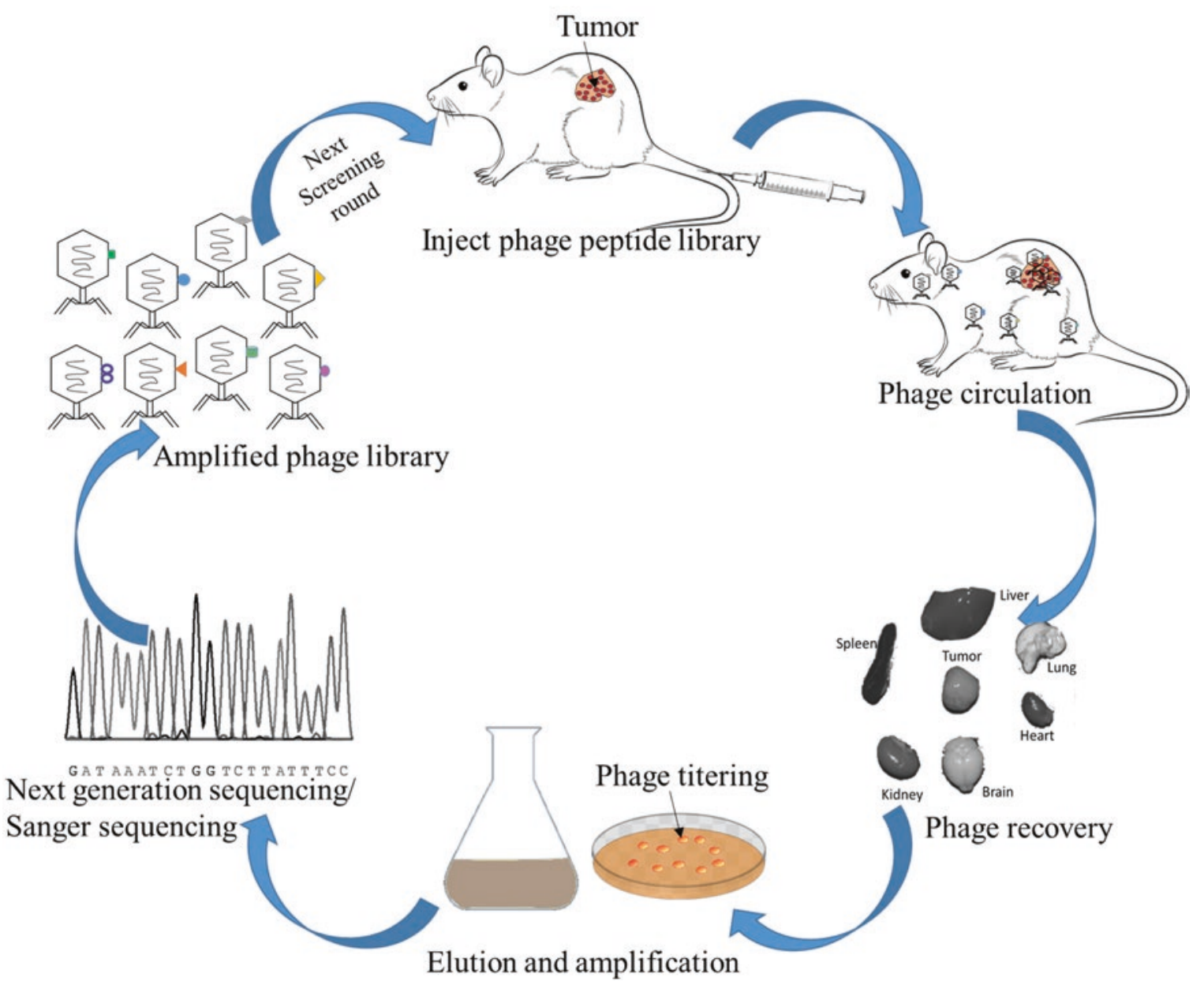

Fig. 1 In vivo peptide phage biopanning. The genome of T7 bacteriophage is modified to express foreign peptides on the capsid of bacteriophage particles as C-terminal fusions of coat protein 10A of a bacteriophage. A peptide library is injected through the tail vein of tumorbearing mice. Ten $\min$ to $1 \mathrm{~h}$ after injection, the mice are perfused through the heart to remove background unbound phages, and organs are collected for phage amplification in E. coli. The phage recovered from the tumor is amplified and reinjected in mice for additional selection rounds, until enrichment is seen. The phages from each round are subjected to DNA sequencing to identify tumor-targeting candidate peptides

\subsubsection{Agnostic In Vivo Peptide Phage Biopanning}

In the second approach, phage display is applied to living animals to identify homing peptides that preferentially accumulate in tumors [107, 194]. This approach takes into account the complexity and heterogeneity of the living animals and is closer to clinical applications. In vivo phage screening primarily probes the vascular beds, and peptide phages generally do not penetrate vascular walls into the tumor parenchyma [165]. Variations of in vivo biopanning besides "classic" intravenous route include alternative administration routes: trans- or intradermal, intratumoral, intraperitoneal (IP), subcutaneous (SC), and intranasal chosen based on the intended target site and screening stringency, and taking into account the desired downstream applications [19, 39, 82, 201]. For detailed protocols on in vivo phage display, the readers are referred to a chapter in the Methods in Enzymology [194]. Briefly, independent of the administration route, the phage particles will be typically allowed to circulate for $10 \mathrm{~min}$ to $1 \mathrm{~h}$, followed by perfusion of the circulatory system with a physiological buffer, such as phosphate-buffered saline, to remove the background of the unbound phages (in screens for internalizing peptides, the perfusion is carried out using a low-pH buffer that inactivates target bound noninternalized phages). The target organs and control organs are then collected for assessment of the titer of the recovered phages and amplified for the next round biopanning. These screening rounds are repeated until enrichment is achieved in the tumor tissue. In typical screens under medium-high stringency conditions, during each round of 
selection the diversity of peptide libraries collapses $\sim 4$-fold. Recently, our and other labs have started using nextgeneration sequencing of the peptide-coding segment of the phage genome and comprehensive assessment of peptide phage biodistribution patterns (custom analysis tool available at http://canbio.ut.ee) $[82,126]$. The ability of candidate peptide phages from in vivo biopanning screens to home to intended targets is further validated in individual peptide phage biodistribution studies or in "in vivo play-off assay." In play-off experiments, equimolar mix of candidate and control phages is administered in mice, followed by the assessment of representation of phages in control and target organs to evaluate the robustness and specificity of homing. The distinct advantage of the play-off experiment compared to individual phage homing tests is that they are internally controlled and interanimal variability does not affect the outcome. After validation of peptides in the phage display format, the promising peptides are synthesized and tested for homing as fluorescent monomeric peptides and as targeting ligands of various payloads (drugs, imaging agents, and nanoparticles).

The target receptors of homing peptides need to be established for understanding the mechanistic details of homing and potential bioactivity, for improvement of the efficiency of targeting and delivery, and for development of improved affinity ligands. Affinity chromatography is widely used technique for peptide receptor identification [127]. The peptides immobilized on solid matrices are incubated with tumor tissue and cell lysate for allowing target receptors to bind to the immobilized peptide. The matrix complex is extensively washed and the target receptor is eluted with excess of free peptide. The elutes are analyzed by SDS-PAGE and silver staining, followed by receptor identification by mass spectrometry (MS) [192]. The candidate receptors are then validated using biochemical, immunological, and cell-based assays to confirm the peptide-receptor binding. Besides affinity chromatography, other methods such as chemical cross-linking and enzyme-based proximity labeling (with BioID and hydrogen peroxidase) can be used [24, 37, 79, 97]. However, false-positive receptor identification remains a significant problem in all the receptor hunting technologies.

\subsection{Docking-Based Tumor Homing Peptides}

\subsubsection{Integrin-Targeting Homing Peptides}

Already more than 3 decades ago, Ruoslahti and colleagues discovered that RGD tripeptide represents a minimal cellbinding sequence in extracellular matrix protein fibronectin (FN) [148, 149]. The identification of the RGD peptide paved the way for the discovery of many more RGDcontaining extracellular matrix proteins such as vitronectin, type I collagen, fibrinogen, von Willebrand factor, osteopontin, and platelet protein gp HIb/lIIa. Ruoslahti and coworkers were also the first to identify transmembrane integrins as receptors for extracellular RGD-containing proteins and synthetic RGD peptides [155, 156, 170]. The integrin family includes 24 structurally related heterodimeric transmembrane proteins consisting of $\alpha$ and $\beta$ subunits that act as receptors for ECM proteins, including TGF- $\beta$, laminin, vitronectin, fibronectin, fibrinogen, and collagens [51]. The RGD-binding site of integrins is formed by sequences from both $\alpha$ and $\beta$ subunits. Over the years, multiple RGDderived peptides and RGD-mimetic integrin ligands have been developed for targeted delivery of therapeutics, imaging agents, macromolecules, and nanocarriers. One such example, Cilengitide - c(RGDf-NMeVal) a potent antagonist of $\alpha v \beta 3$, has been tested in glioblastoma multiforme phase-III clinical study by Merck [49, 51]. However, the trial failed to produce overall survival endpoints in GBM patients. Other RGD-containing peptides (e.g., cRGDfV, cRGDfK, and RGD4C) have been preclinically tested $[102,125]$. The $\alpha v \beta 3$ and $\alpha v \beta 5$ integrins play important roles in angiogenesis [172]. The variants of RGDs peptide, including CRGDfK, cRGDfC, CRGDyK, have been tested in design of $\alpha v \beta 3$ and $\alpha v \beta 5$ integrin targeted drug delivery systems in multiple cancers in vivo. The RTDLXXL peptide motif identified by a phage display binds with nanomolar affinity to $\alpha v \beta 6$ integrin - another member of the integrin family overexpressed in many solid tumors [99]. These RGD-based integrin targeting probes have been used for noninvasive cancer imaging and for the development of biomarkers. RGD based positron emission tomography (PET) ${ }^{18} \mathrm{~F}$ - and ${ }^{68} \mathrm{Ga}$ probes were developed and tested for imaging of solid cancers. Examples are ${ }^{18} \mathrm{~F}$-fluciclatide [177] and ${ }^{68} \mathrm{Ga}$-linked bombesin-RGD [213] used in probing prostate tumors and metastases, ${ }^{124}$ I-labelled RGD nanoparticle used in multimodal PET/ optical theranostic probes [25], radiolabeled A20FMDV2 peptide explored in targeting $\alpha v \beta 6$ in breast cancer [96], and NIR light-guided surgery in preclinical models ovarian carcinoma and gastric cancer [40, 91].

\subsubsection{Aminopeptidase N Targeting Homing Peptides}

Asn-Gly-Arg (NGR) homing motif first emerged in cell-free peptide phage biopanning against $\alpha 5 \beta 1$ integrins that resulted in the selection of different RGD-containing peptides and NGR motif-containing peptides [14, 101]. The relevance of the NGR motif for systemic delivery was confirmed by an in vivo phage display study in mice bearing human breast carcinoma xenografts that led to identification of tumor vasculature-homing peptide CNGRCVSGCAGRC [14, 142]. The minimal cyclic peptide CNGRC inhibited the accumulation of the other NGR motif peptides [14]. NGR does not compete with RGD peptides, suggesting both binds 
to different receptors. The NGR motif showed specific binding to aminopeptidase N (APN/CD13), a membrane-bound metallopeptidase involved in angiogenesis, cell migration, and proliferation, antigen presentation, and regulation of the activity of hormones and cytokines [119, 142]. The NGR peptide showed a high affinity to CD13 expressed on the surface of the endothelial cells of tumor blood vessels [14]. The CD13 is overexpressed in angiogenic tumor vessels and under other pathologic conditions, such as inflammation and retinal disorders [27, 31, 33, 65, 204].

The NGR peptide is widely used as an affinity ligand to deliver anticancer drugs and imaging agents. Treatment of mice bearing orthotopic neuroblastoma, prostate, lung, and ovarian cancer xenografts with NGR-guided doxorubicin (DOX) liposomes resulted in dramatic destruction of the tumor vasculature and prolonged survival of the mice [60, 144, 145]. CNGRC fusion to proapoptotic ${ }_{\mathrm{D}}\left(\mathrm{KLAKLAK}_{2}\right.$ peptides rendered the peptide selectively toxic for angiogenic endothelial cells [56]. The NGR peptides have been conjugated to different classes of cargoes such as nanoparticles, cytotoxic drugs, therapeutic proteins, proapoptotic peptides, viral particles, DNA/RNA, and imaging agents. The recombinant NGR-hTNF $\alpha$ cytokine, in combination with chemotherapeutics, has been tested in preclinical and clinical trials (phase I, II and III) showing promising efficacy [62, 63, 118]. NGR-hTNF $\alpha$ at low dose in combination with chemotherapeutic drugs (DOX, cisplatin; gemcitabine; pemetrexed, oxaliplatin) exerts synergistic effects and enhanced drug penetration, resulting in increased therapeutic response in various solid tumors in patients [119].

\subsubsection{P32/qC1qR Targeting}

The p32 is a small acidic mitochondrial chaperone that is expressed intracellularly in resting adult cells and displayed on the surface of tumor endothelial cells (blood and lymphatic), tumor cells, and tumor macrophages. First p32 targeting peptide, Lyp-1 (CGNKRTRGC), was originally identified in an in vivo screen for peptides that home to lymphatic vessels in breast tumors [109]. The Lyp-1 binds p32 in tumor cells, tumor-associated macrophages, and tumor lymphatics. It recognizes lesions from the premalignant lymphatic niche to fully metastatic tumors. Even though many normal cells express intracellular p32, it is a tumor-specific target as only the cell surface p32 present on malignant cells is accessible for cell-impermeable p32 ligands. Extracellular p32 ligands are taken up by the p32-displaying cells and become associated with the mitochondria, suggesting trafficking between the cell surface and the mitochondria. Importantly, p32-binding ligands have an inherent pharmacological activity and display an apoptosis-inducing activity on cultured p32-displaying tumor cells.

Recently we identified a novel ligand of p32, TT1 peptide (active both as a disulfide-bridged cyclic CKRGARSTC and as linearTT1, AKRGARSTA) [137, 178]. Compared to parental LyP-1 peptide, the TT1 peptide showed improved affinity in cell-free binding studies and excellent homing in vivo. In the same study, we reported a high-throughput screening of compound libraries that led to identification of a low molecular weight p32-targeting ligand (Cambridge compound \#4014008) that shares the binding site with LyP-1 [137, 178].

Lyp1 and TT1 compete with each other for p32 binding and both contain cryptic tumor-penetrating C-end Rule (CendR) elements (see below). Homing and extravasation of TT1 relies on multistep homing and tumor penetration pathway: first, it binds to $\mathrm{P} 32$ protein on cell surface, followed by processing by a tumor-related protease, urokinase-type plasminogen activator (uPA), to expose conditional C-terminal CendR motif (AKRGAR) with newly acquired ability to bind to NRP-1 and to trigger vascular exit and internalization of the peptide [147, 173]. Similarly, Lyp-1 has been shown to be capable of penetrating tumors and to accumulate in the extravascular tumor parenchyma. Both P32 targeting peptides have been evaluated in numerous preclinical studies. The p32 targeting peptides have been evaluated in preclinical efficacy studies as targeting ligands of paclitaxel-albumin nanoparticles, DOX-loaded liposomes and polymersomes. LyP-1 peptide was originally identified as a lymphatic endothelial cell-homing peptide. Besides LyP-1, there are other lymphatic targeting peptides, such as AGR (CAGRRSAYC) and REA (CREAGRKAC) peptides shown to exhibit tumor tissue and stage-specific lymphatic homing patterns [114].

\subsubsection{Homing Peptides for Targeting Fibrin- Fibronectin Complexes}

Tumors as "wounds that do not heal" are known to contain a meshwork of clotted plasma proteins in the tumor stroma and in the walls of the tumor blood vessels. This tumor ECM complex supports tumor cell migration, survival, and proliferation and could serve as a potential target for affinity probes in the tumor microenvironment. Ruoslahti lab has used in vitro peptide phage library on plasma clots and identified peptides CGLIIQKNEC (CLT1) and CNAGESSKNC (CLT2) that specifically bind to clotted plasma proteins in tumors [150]. In mouse models of orthotopic breast cancer, fluorescein (FAM)-conjugated CLT1 and 2 peptides specifically homed to tumor ECM, whereas nonmalignant tissues remained negative. CLT1 peptide conjugated to PEG-PLA nanoparticles (CNP) loaded with paclitaxel significantly prolonged the survival in glioma-bearing mice [212]. CLT1 peptide conjugated to Gd-DTPA for molecular imaging of fibronectin-fibrin complexes in tumor tissue with magnetic resonance imaging (MRI). CLT1-(Gd-DTPA) demonstrated significant tumor contrast enhancement for at least $60 \mathrm{~min}$ in mice bearing HT-29 human colon carcinoma xenografts [208]. 
CREKA is another clotted plasma protein binding peptide identified by in vivo phage display screen with tumor-bearing MMTV-PyMT transgenic breast cancer mice [181]. CREKA peptide homes to extracellular meshwork in the tumor stroma of MMTV-PyMT tumors and MDA-MB-435 human breast cancer xenografts and also accumulated in malignant but not normal blood vessels [3, 181]. CREKA pentapeptide has a free sulfhydryl group not required for binding activity that is available for conjugation with nanoparticles, drugs, and imaging agents. CREKA-coated iron oxide nanoworms (NWs) act as a self-amplifying nanoparticle-delivery system that causes tumor-specific clotting to trigger binding of additional clot-homing particles. Systemic CREKA-NWs can be used for T2 MRI-based imaging and therapy of orthotopic human prostate cancer modeled in mice [4]. Creka-liposomes loaded with DOX (CREKA-Lipo-Dox), and platelet inhibitor ticagrelor (CREKA-Lipo-T) showed enhanced antitumor and antimetastatic efficacy in metastatic breast cancer model $[90,215]$. Systemic treatment of breast tumor mice with CREKA peptide fusion with the truncated extracellular domain of tissue factor (tTF-CREKA) induced tumorselective intravascular thrombosis, reduced tumor blood perfusion, and resulted in inhibition of tumor growth. tTF-CREKA selectively blocks the tumor blood supply and can be applied to different types of solid tumors [179]. In various other diseases, complexes of CREKA with Fe3O4PLGA-PFH and SPION nanoparticles have been used for invasive multimodal molecular imaging (ultrasound and photoacoustic imaging) and therapy in thrombosis and myocardial ischemia models in mice and rats [185, 218].

\subsubsection{Homing Peptides for Stage-Specific Tumor Targeting}

The tumor stage-specific targeting peptides recognize neoplastic vascular signatures during different stages of tumor development $[110,164,194]$. A panel of such peptides and motifs were identified using in vivo phage display in the RIP1-Tag2 transgenic model of islet cell carcinoma, squamous cell carcinomas, pancreatic neuroendocrine tumor, and human MDA-MB-435 breast cancer [73, 93]. The peptides KAA (sequence: CKAAKNK) and KAR (sequence: CKGAKAR) were found to home preferentially to the established tumor over angiogenic islets. The peptides RSR (sequence: CRSRKG) and EYQ (sequence: CEYQLDVE) showed angiogenic islet-specific homing, with little or no binding to tumor vessels and to normal islets of control organs. Finally, VGVA (sequence: FRVGVADV) and RGR (sequence: CRGRRST) were found to home to vasculature of both established tumors and angiogenic islets [93].

Stage-specific tumor-homing peptides have been used successfully for preclinical imaging and efficacy studies. For example, tumor-targeting peptide KAA-guided nanoparticles loaded with anticancer drug gemcitabine showed robust homing to neovessels of pancreatic cancer in mice and therapeutic efficacy [198].

\subsubsection{Malignant Extracellular Matrix Targeting Peptides}

The extracellular matrix (ECM) is an important element of tissue architecture that provides structural and functional support for its cellular constituents. ECM is dynamic and adapts to changes in the microenvironment by remodeling its composition and topology, resulting in tissue and diseasespecific ECM signatures $[59,86]$. Compared to cell surfacehoming peptide receptors, tumor ECM components are more abundant, thus providing a higher binding capacity $[87,115]$. Notably, the oncofetal fibronectin Extra Domain-B (FN-EDB) and Tenascin-C (TNC) are overexpressed in many solid tumors and nearly absent in nonmalignant tissues $[34,140,180]$. There are various monospecific affinity ligands identified over the years to target FN-EDB or TNC, including for FN-EDB (ScFV L19 [132], ZD2 [67]) and TNC (ScFV G11 [180], TNC aptamer [47], and TNCbinding FHK peptide [98]). The FN-EDB and TNC antibodies (FN-EDB ScFV L19, TNC-C ScFV G11, F16, and 81C6) are used for precision delivery of cytokines (e.g., IL2 and TNF) and radionuclides [105, 186]. These ECM protein targeting ligands demonstrated their potential advantage in tumor targeting and delivery in various solid tumors.

Recently, we used cell-free screening to identify a 12 amino acid peptide, PL1 (sequence: PPRRGLIKLKTS), that recognizes two tumor associated ECM molecules: FN-EDB and tenascin-C C domain (TNC-C) [115]. The simultaneous affinity targeting of the two targets with the same affinity ligand allows for more uniform tumor targeting by overcoming the spatiotemporal heterogeneity in expression of target molecules and alleviating issues related to the limited number of available receptors for targeting ligands [81]. Another advantage of a bispecific peptide is that it helps to achieve synergistic targeting and delivery compared to monospecific targeting. The intravenously injected PL1 nanoworms (NWs) and silver nanoparticles (AgNPs) showed robust accumulation in a panel of glioblastoma and prostate carcinoma xenografts modeled in mice [115]. The PL1 nanoparticle accumulation was at least partially angiogenesis-dependent, as nonmalignant angiogenic neovessels induced by VEGF-overexpression we also found to take up PL1-NWs. The homing of nanoworms was dependent on both TNC-C and FN-EDB targeting, as functionblocking antibodies to either target alone decreased the homing, and a cocktail of both antibodies blocked the homing. Further, the experimental therapy of glioblastoma mice with proapoptotic nanoworms resulted in extended survival without apparent toxicity and PL1-NWs bound to the cryosections of clinical glioblastoma, suggesting translational relevance [115]. 


\subsubsection{Hyaluronan Targeting Peptides}

Hyaluronic acid (HA) is a nonsulfated glycosaminoglycan (GAG) that is negatively charged and contributes to the mechanical integrity of the ECM network with complex biological functions ranging from matrix organization, cell adhesion, migration, angiogenesis, morphogenesis, wound healing, and inflammatory responses to cancer metastasis $[182,214]$. Recently, we reported intraperitoneal in vivo phage display screens using the T7 CX7C library in mice with peritoneal carcinomatosis (xenografts of gastric and colon carcinoma) [82]. This screen led to identification of IP3 (sequence: CKRDLSRRC) 9-residue cyclic peptide that contains a HA-binding motif. Fluorescein-labeled monomeric IP3 peptide bound to immobilized HA in vitro and the intraperitoneal IP3-coated silver nanoparticles and free fluorescent IP3 peptide showed preferential accumulation in peritoneal nodules of carcinomas of colon, gastric, and ovarian origin [82].

Moreover, a synthetic 42-amino acid peptide, BH-P (sequence: CNGRCGGRRAVLGSPRVKWTFLSR GRGGRGVRVKVNEAYRFR), derived from human brain HA binding protein, was reported to bind to HA and possess antitumor activity both in vitro and in vivo [116].

\subsubsection{Tumor-Associated Macrophage Targeting Peptides}

Tumor-associated macrophages (TAMs) are part of the innate immune system and have been associated with poor prognosis in many types of solid tumors. TAMs promote angiogenesis and tumor cell growth, metastasis, and contribute to the immunosuppressive environment. TAMs are categorized into M1 (M1 TAMs) and M2 macrophages (M2 TAMs). After chemotherapy, M2 TAMs accumulate around blood vessels in tumors, where they promote tumor revascularization and relapse [78, 151]. Pro-tumoral M2-like TAMs in progressing neoplasms typically express characteristic surface molecules, such as various scavenger receptors, for example, the hemoglobin scavenger receptor CD163, scavenger receptor A (SR-A), and mannose receptor-1 (also known as CD206) [18, 174]. These cells secrete growth factors, such as epidermal growth factor (EGF), which stimulates proliferation of carcinoma cells and cytokines, and IL-1, that promote the accumulation of tumor cells at distant sites $[38,136,205]$. Due to the central role that TAMs play in tumor progression and therapeutic resistance, they are recognized as a translationally relevant target for cancer immunotherapy and triggered efforts aimed at their elimination or reprogramming. Protumoral TAMs are not a single uniform population; instead, they are composed of multiple distinct subpopulations with overlapping features depending on a variety of external factors. Defining, differentiating, and targeting these subsets remains a challenging work-in-progress. Over the years a number of affinity ligands to target TAMs have been identified and several compounds are being evaluated in clinical trials. However, these targeting ligands are promiscuous and target other cells besides TAMs. For example, the peptide IDR RP-182 triggers a conformational change in CD206 on M2-like macrophages that shifts M2-like macrophages toward an M1 phenotype [88]. Another group identified by biopanning on isolated M2 macrophages the M2pep peptide (sequence: YEQDPWGVKWWY) that binds CD11b + Ly6G-F4/80high subpopulation of M2 macrophages. M2pep fusion to ${ }_{\mathrm{D}}(\mathrm{KLAKLAK})_{2}$ was able to selectively reduce the M2-like TAM population, but not the M1 macrophages, in tumor-bearing animals [42].

Recently we reported the identification of a peptide (mUNO, sequence: CSPGAK) that binds to mouse CD206 and homes to M2 TAMs in multiple solid tumor models, suggesting that peptide homing is dependent on the presence of M2 TAMs and not on the tumor type [16, 175]. Systemically administered fluorescein-labeled mUNO homes exclusively to CD206 ${ }^{\text {high }}$ M2 TAMs in metastatic breast cancer, with no accumulation in tumor-free liver [16, 175]. Importantly, M2 TAMs internalize FAM-mUNO complexes, suggesting that the peptide can be used for intracellular payload delivery. Molecular dynamics simulations, docking predictions and fluorescence anisotropy studies suggested that on CD206 mUNO binds to a binding pocket between C-Type Lectin Domains-1 and -2 that is not involved in mannose binding. This observation provides an explanation for the improved selectivity of mUNO towards CD206 compared to mannosebased ligands such as Manocept ${ }^{\mathrm{TM}}$.

\subsubsection{Nucleolin Targeting Peptides}

Nucleolin is a multifunctional phosphoprotein found localized ubiquitously in the nucleolus and cytoplasm in healthy cells and is overexpressed in the cell surface of activated cells in many types of solid tumors [26, 191]. Nucleolintargeting F3 peptide (sequence: KDEPQRRSARLSAKPAPPKPEPKPKKAPAKK), identified by biopanning with the cDNA phage library, corresponds to 31-aminoacid fragment of the high mobility group protein 2 (HMGN2). The F3 peptide interacts with cell surface-expressed nucleolin on endothelial cells [152] and is able to translocate into the nucleus of tumors cells of different origin [15, 154]. Fluorophore-labeled F3 peptide was found to internalize and translocate in the nuclei in human myeloid leukemia cells and in human breast cancer cells. The cellular uptake of F3 is specific as blocking F3-nucleolin interaction with antibodies abolished binding and internalization. The F3 peptide has been used as a tumor-targeting ligand for nanoparticles, chemotherapeutics, and radiotherapeutic agents $[53,95,154,157,216]$.

Another nucleolin binding peptide, HB-19 pseudopeptide $\left(5\left[\mathrm{Kpsi}\left(\mathrm{CH}_{2} \mathrm{~N}\right) \mathrm{PR}\right]-\mathrm{TASP}\right)$, originally identified as a potent inhibitor of HIV-1 infection, inhibits angiogenesis and 
growth of human breast tumor xenografts orthotopically modeled in mice, and reduces metastasis while causing no toxicities in normal tissues [52, 55, 134].

\subsubsection{Epidermal Growth Factor Receptor Targeting Peptide}

Members of the EGFR family of receptor tyrosine kinases, including HER1 (EGFR, ErbB1), HER2 (Neu, ErbB2), HER3 (ErbB3), and HER4 (ErbB4), are involved in a wide range of processes, including cell growth, proliferation, survival, migration, and tissue invasion and are overexpressed in many types of solid tumors including breast, colon and nonsmall cell lung cancer (NSCLC) [129, 217]. FDAapproved modulators of the EGFR pathway include monoclonal antibodies (cetuximab and panitumumab) and small molecular inhibitors (gefitinib, erlotinib, and afatinib).

EBP peptide (sequence: CMYIEALDKYAC) was developed by structural modeling of the natural EGFR ligand, EGF [103]. EBP conjugate with DOX showed enhanced anticancer efficacy and reduced systemic toxicity in orthotopic breast cancer xenograft mice [7, 8]. Liposomes functionalized with another EGFR-targeting peptide, D4 (sequence: LARLLT), were found to accumulate in EGFR expressing xenograft tumor tissues [184]. Recently, another EGFR-targeting peptide, GE11 (sequence: YHWYGYTPQNVI), identified by peptide phage display, was found to bind cultured MDA-MB-231 breast cancer cells and was able to systemically deliver gene/drugs to EGFR overexpressing cancer in vivo [74]. Two additional peptidic affinity ligands to EGFR, KCCYSL, and LTVSPWY, were also discovered by phage display and have proven useful for imaging and targeted drug delivery to EGFR-positive tumors [217]. Finally, two EGFR targeting peptides, EDA (sequence: PgYNPTTYQAha) and disruptin (sequence: SVDNPH), were biologically active and inhibit dimerization of EGFR and downstream signaling [5, 6, 70].

\subsection{Tumor-Penetrating Peptides}

Poor tissue penetration is a serious limitation of drug delivery to tumors, and owing to their size, nanoparticles and antibody drugs, in particular, suffer from this limitation [196]. The barriers are exit from the vasculature and penetration through tumor tissue. The frequently cited "leakiness" of tumor vessels permits passive extravasation, but it is not clear to what extent clinical tumors share this characteristic with the commonly used subcutaneous experimental tumors. Moreover, antiangiogenic treatments cause "normalization" of tumor vasculature [84], making it likely that nanodevices will be deployed with tumors whose vasculature is not leaky. High interstitial pressure in tumors constitutes a major barrier to penetration of extravasated compounds within extra- vascular tumor tissue, as does abundant fibrotic tissue, which is common in tumors such as pancreatic cancer [72, 85, 167].

About 15 years ago, the tumor homing LyP-1 peptide with the ability to target the lymphatic vessels and hypoxic areas in tumors was identified [108]. Systemically administered LyP-1 phage was found to accumulate in macrophages and lymphatic vessels away from blood vessels. In 2009, we identified a family of internalizing and tumor-penetrating C-end Rule or CendR peptides that contain a $\mathrm{R} / \mathrm{KXXR} / \mathrm{K}$ recognition motif that needs to be carboxyterminally exposed for activity [188, 192]. The CendR receptor NRP-1 is a coreceptor of semaphorins, VEGF family members, and multiple other growth factors that play essential roles in vascular biology and progression of solid tumors [61, 128]. CendR tumor-penetrating peptides (TPP) activate an endocytic transport pathway related to but distinct from macropinocytosis by engaging a complex process that involves binding to a primary, tumor-specific receptor, a proteolytic cleavage, and binding to a second receptor (Fig. 2). The binding to the second receptor NRP-1 activates the transport pathway [139, 192]. The pathway is distinct from known endocytosis pathways and is partially characterized [139].

The first TPP to be discovered was iRGD peptide (sequence: CRGDKGPDC or CRGDRGPDC) [188] that is clinically developed by San Diego-based DrugCendR Inc. as CEND-1. The iRGD peptide combines the tumor homing ability of the RGD motif with conditional proteolytically activatable CendR tissue penetration switch, rendering it highly potent and tumor-specific. First, the RGD sequence motif drives the recruitment of the peptide to $\alpha v \beta 3$ and $\alpha v \beta 5$ integrins on the surface of tumor angiogenic vascular endothelial cells and tumor cells. Second, a tumor-derived protease cleaves iRGD to generate fragment with C-terminally exposed CendR motif (CRGDK/R). The truncated peptide loses integrin-binding activity but acquires ability to interact with b1 domain of tissue penetration receptor NRP-1. Interaction with NRP-1 then activates a CendR pathway (endocytotic/exocytotic transport pathway) for enhanced transport of drugs into tumors [188]. The $\alpha v \beta 3$ and $\alpha v \beta 5$ requirement and involvement of a tumor-derived proteolytic convertase render iRGD activation tumor-specific. The iRGD peptide can carry a payload as large as a nanoparticle deep into tumor tissue. Surprisingly, the cargo does not have to be attached to iRGD; compounds co-administered with iRGD are also transported from the vasculature into the tumor parenchyma [189]. Co-injection of iRGD has resulted in enhanced tumor penetration and accumulation of each of the several compounds tested, and the phenomenon has been observed in a large number preclinical tumor models (transgenic models, xenograft and syngeneic implants of breast, pancreatic, prostate, and ovarian tumors), including metastases [189]. iRGD combination treatment does not affect the extent of the side effects, such as cardiomyopathy caused by 


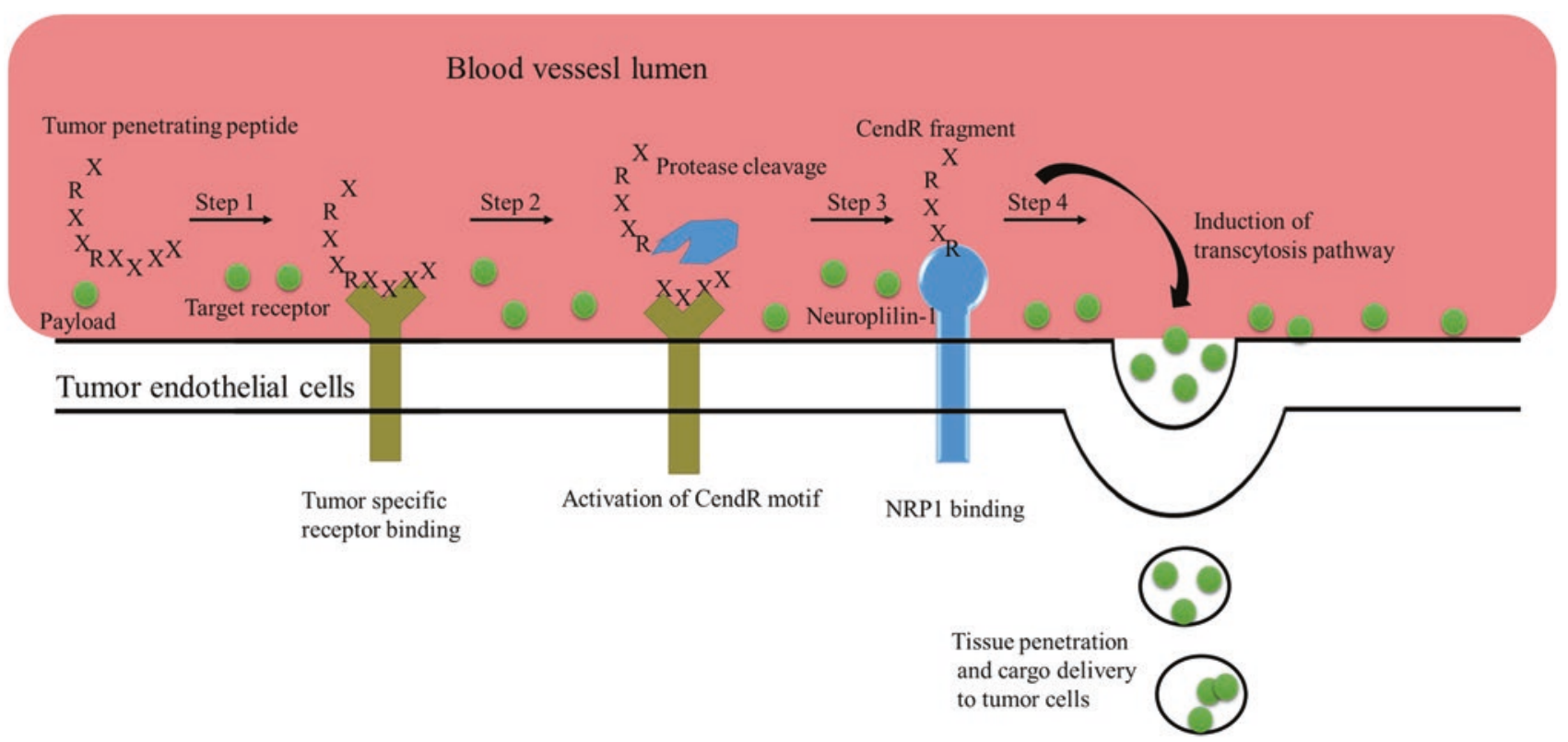

Fig. 2 CendR trans-tissue transport pathway. The TPP cell and tissue penetration pathway is based on a three-step process. First, the peptide is recruited to primary tumor-specific target receptors (e.g., $\alpha v \beta 3 / \alpha v \beta 5$ integrin in the case of iRGD; $\mathrm{p} 32 / \mathrm{gC} 1 \mathrm{qR}$ in the case of LyP-1 and

doxorubicin. Thus, iRGD broadens the therapeutic index of cancer drugs.

\subsubsection{Novel Tumor-Penetrating Peptides}

A defining feature of the CendR system is that the activity of the cell and tissue-penetrating CendR motif is strictly position-dependent and requires carboxy-terminal exposure of consensus element R/KXXR/K. Proteolytic enzymes mediate diverse processes such as extracellular matrix remodeling, immunity, development, protein processing, cell signaling, and apoptosis [54]. Of approximately 700 peptidases of the human degradome, nearly $1 / 4$ have cleavage specificities compatible with CendR exposure. Many of the proteases are expressed and secreted in a tissue or disease specific pattern. Incorporation of the CendR element in the context of a protease recognition and cleavage site can be applied for the activation of tissue-penetrating activity at a target site. CendR peptides of novel specificities can be constructed by combining CendR elements with protease cleavage sites and vascular homing modules - either by rational design or screening-based approaches. We have validated the concept by designing cryptic CendR peptides that are activated by urokinase-type plasminogen activator (uPA) [29]. The association of the UPA activity with tumorigenesis and its strong substrate selectivity make it an attractive candidate for protease-activated cancer targeting [29, 124, 182]. Our designer uPA-dependent tumor-penetrating peptide, uCendR, contains the following modules (starting from the N-terminus): a tandem RXXRXXR CendR element in the
TT1). In the second step, the peptide is cleaved by proteases to unmask the cryptic CendR element $(\mathrm{R} / \mathrm{KXXR} / \mathrm{K})$ at the $\mathrm{C}$-terminus. Finally, the CendR motif interacts with neuropilin-1 (NRP-1) to trigger vascular and tumor tissue penetration

context of uPA cleavage site, and a vascular homing motif CRGDC. The uCendR peptide is recruited to tumor endothelial cells and tumor cells, followed by cleavage by uPA and NRP-1 dependent penetration into tumor tissue.

Besides angiogenic integrins, other primary receptors have been used for TPP design. For example, the NGR tripeptide tumor homing motif that targets aminopeptidase $\mathrm{N}$ (CD13) was embedded in iRGD-derived cyclic peptide scaffold, resulting in internalizing iNGR peptide (sequence: CRNGRGPDC) [9]. The iNGR peptide was found to home to CD13 in tumor vessels and, unlike parental NGR peptide, extravasate, and penetrate deep into the tumor parenchyma. For iNGR peptide-coupled paclitaxel nanoparticles and coadministered DOX, improved biodistribution and antitumor efficacy were observed [211, 219]. In recent studies, the iNGR peptide has been used to potentiate the tumor homing of lanthanide nanoclusters [206], and for targeted co-delivery of chemotherapeutic drugs and photosensitizer to treat treatment-resistant cancer [89].

The p32-targeting LyP-1peptide (sequence: CGNKRTRGC), discussed above, contains a cryptic CendR element, shows robust extravasation and parenchymal penetration in tumor tissue, and is likely to use the CendR pathway for tumor homing and penetration. The truncated version of LyP-1 with exposed CendR motif, tLyP-1 (sequence: CGNKRTR), binds to p32 and internalizes in cells through the CendR pathway [163]. Compared to parental peptide, tLyP-1 improves extravasation of a co-injected nanoparticle into the tumor tissue. As it uses a different primary receptor 
that iRGD, it may act as complementary peptide for iRGD [163]. Recently, we have identified another P32-targeting cyclic peptide TT1 (sequence: CKRGARSTC) [137]. Similar to Lyp-1 peptide, TT1 (and its linear variant, linTT1, AKRGARSTA) showed homing and internalization in different tumor models in vivo [80, 173, 178]. TT1 and LinTT1 peptides can be activated by cleavage by tumor-derived uPA to carboxyterminally expose the AKRGAR active CendR fragment [178]. Both tLyP-1 and TT1 peptides have applications for targeted drug delivery of anticancer drugs or imaging agents to $\mathrm{p} 32$ overexpressing cancers.

\subsubsection{Translational Development and Perspectives of TPP Technology}

TPP enables specific tumor targeting, increasing the drug accumulation in the tumor, and promoting even drug distribution throughout the tumor. A unique property of TPPs is that the peptides can be used without the need to couple the drug to the targeting agent [189]. The TPP acts through a three-step process involving binding to a receptor present only in tumors, at the surface of tumor vessels, in particular, a subsequent proteolytic cleavage, and transfer to another receptor. The binding of processed TPP to NRP-1 activates a vesicular transport pathway (CendR pathway) that is meant to carry nutrients into tissues [139]. The current hypothesis is that a drug co-administered with TPPs will be transported into tissue as if it were a nutrient. Because TPPs activate the transport pathway only in cancerous tissue, the drug will only accumulate and spread within tumors, not in normal tissues, and the result is enhanced anticancer activity without an increase in side effects. There are currently nearly 200 publications in the scientific literature on applications of prototypic TPP iRGD and the CendR technology for improving the biodistribution and potentiation of a variety of compounds ranging from low molecular weight compounds to macromolecules and nanoparticles, that come from a large number of laboratories located all over the world. In animal studies, iRGD has been shown to enhance the efficacy of kinase inhibitors, cytokines, antibodies, nucleic acid-based therapeutics siRNA, and even anticancer immune cells [209, 220]. The increase in the activity of anti-cancer agents provided by iRGD has been shown for a variety of tumor types. Thus, iRGD/TPP can potentially become a platform technology able of enhancing the activity of a variety of treatment (and imaging) modalities aimed at solid tumors. Alternatively, as the boost in anticancer activity is tumor specific, iRGD has the potential to reduce systemic toxicity of treatments.

The lead indication, currently under Phase I clinical development, is iRGD-enhanced gemcitabine/abraxane therapy for treatment of pancreatic ductal adenocarcinoma (https://clinicaltrials.gov/ct2/show/NCT03517176). Clinical validation of $\mathrm{iRGD}$ as a method to enhance antitumor activity of co-administered gemcitabine and abraxane is expected to lead to broader efforts to combine iRGD and other TPPs that are currently in the preclinical development phase with anticancer agents. The use of iRGD will be a therapeutic booster that will not require patients to be removed from standard of care therapy. iRGD-based imaging approaches are also being developed that will serve as functional biomarkers of iRGD-enhanced tumor uptake in the clinic. Preclinical studies with iRGD and contrast agents show robust signals for iRGD-mediated tumor uptake and penetration of the contrast agent in mouse models [193, 220], indicating that iRGD-based imaging approaches could be used for patient stratification. The iRGD peptide is straightforward to manufacture and should provide a cost-effective therapeutic approach to improve the tumor localization, distribution, and efficacy of co-administered therapeutics.

\section{Conclusions and Perspectives}

Affinity targeting with tumor-homing peptides is an established technology that can be used to achieve improved selectivity and efficacy of anticancer drugs to widen their therapeutic index. The current arsenal of tumor-homing peptides allows targeted drug delivery with great precision to various components in malignant tissues: blood and lymphatic vascular trees, extracellular matrix, immune cells, and fibroblasts (Fig. 3). Translational potential of homing peptides is further strengthened by their small size, low immunogenicity, and biocompatibility. Peptides are usually not species-specific as they target functionally important binding pockets on target molecules, and these sites are highly conserved among species. This circumstance increases the translational potential of peptides. Finally, vascular-homing peptides can be readily identified in an unbiased manner by in vivo phage display.

Among tumor-homing peptides, tumor-penetrating CendR peptides hold a great promise. Activating the CendR pathway in a tumor-specific manner provides a specific way of increasing the activity of conjugated and co-administered anticancer drugs and of enhancing tumor imaging. iRGD and other TPP (tLyP-1, iNGR)-based approaches are the only technologies that enable specific tumor targeting, promote even drug distribution throughout the tumor, and can be used without the need to couple the drug to the targeting agent. Thus, the tumor-penetrating CendR peptides represent a potentially transformative advance in cancer treatment. iRGD is currently undergoing clinical testing, and the next few years are expected to show to what degree the promising preclinical data on TPP are validated in clinical settings.

Refinement of intratumoral precision delivery strategies using the homing peptides is expected to be an important frontier in progress towards more efficacious and more tolerable anticancer strategies. Intratumoral precision delivery 


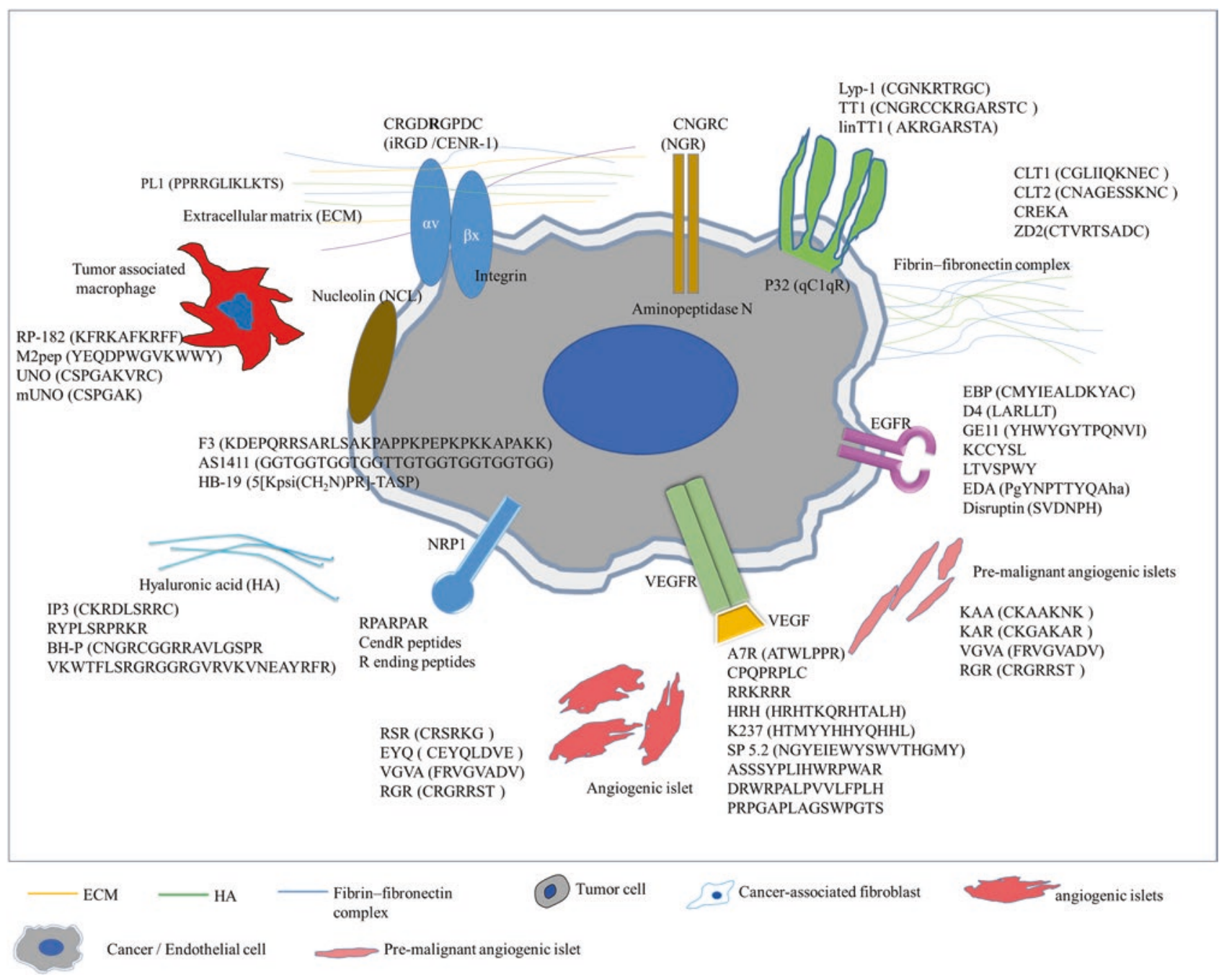

Fig. 3 Tumor-homing peptides. Overview of tumor-homing peptides and their receptors

may be particularly relevant in the context of immunotherapies, where modulation of precisely defined cell populations (e.g., M2 TAMs, Tregs) may result in profound modulation of therapeutic response. For example, iRGD homes to Treg cells, the main immunosuppressive cells in tumors, and iRGD anticancer drug combination may be able to selectively destroy these cells, while leaving the antitumor immune cells intact.

Homing peptides can have a transformative impact on the clinical development of precision-guided nanodrugs and imaging agents. Where 15 passively targeted nanocarriers are FDA-approved for clinical use, none of the actively targeted nanodrugs have advanced past clinical testing and obtained approval [161]. Most of the nanodrugs approved to date have, compared to conventional drugs, demonstrated reduced toxicity and better tolerability rather than improved efficacy. Currently, there are more anticancer nanodrugs in clinical trials than any other drug classes [162]. In numerous preclinical studies, the application of the affinity targeted nanomedicine results in a high tumor concentration of drugs due to a combination of tumor receptor-dependent uptake and EPR-based accumulation. For example, DOX-loaded anti HER2-guided immunoliposomes showed significantly superior therapeutic results compared to control nontargeted liposomal DOX and free DOX [100, 141].

The final issue that needs to be addressed in the coming years is the limiting number of receptors for targeting ligands - a serious limitation of affinity targeting strategies that limits the capacity of the targeting systems [81]. This issue can be alleviated by using the peptide receptors that are highly abundant and accessible (such as ECM components) or combining targeting peptides of different binding specificities (multitargeting). Additional advantage of multitargeted tumor-homing peptides is that they can be used to overcome uneven tissue distribution of individual targets for more uniform payload delivery. In conclusion, peptide targeted drug therapies will continue to contribute a significant innovation in targeted nanomedicine development for maxi- 
mal treatment safety and efficacy for cancer therapy and diagnosis in the near future.

Acknowledgment T. Teesalu was supported by the European Regional Development Fund (Project No. 2014-2020.4.01.15-0012) and Estonian Research Council (grants PRG230 and EAG79).

\section{References}

1. Abdollahpour-Alitappeh, M., Lotfinia, M., Gharibi, T., et al (2019). Antibody-drug conjugates (ADCs) for cancer therapy: Strategies, challenges, and successes. Journal of Cellular Physiology, 234, 5628-5642.

2. Abedon, S. T., Kuhl, S. J., Blasdel, B. G., \& Kutter, E. M. (2011). Phage treatment of human infections. Bacteriophage, 1, 66-85. https://doi.org/10.4161/bact.1.2.15845.

3. Agemy, L., Kotamraju, V. R., Friedmann-Morvinski, D., et al. (2013). Proapoptotic peptide-mediated cancer therapy targeted to cell surface p32. Molecular Therapy, 21, 2195-2204. https://doi. org/10.1038/mt.2013.191.

4. Agemy, L., Sugahara, K. N., Kotamraju, V. R., et al. (2010). Nanoparticle-induced vascular blockade in human prostate cancer. Blood, 116, 2847-2856. https://doi.org/10.1182/ blood-2010-03-274258.

5. Ahsa, A., Ramanand, S. G., Bergin, I. L., et al. (2014). Efficacy of an EGFR-specific peptide against EGFR-dependent cancer cell lines and tumor xenografts. Neoplasia (United States), 16, 105114. https://doi.org/10.1593/neo.14182.

6. Ahsan, A., Ray, D., Ramanand, S. G., et al. (2013). Destabilization of the epidermal growth factor receptor (EGFR) by a peptide that inhibits egfr binding to heat shock protein 90 and receptor dimerization. The Journal of Biological Chemistry, 288, 26879-26886. https://doi.org/10.1074/jbc.M113.492280.

7. Ai, S., Duan, J., Liu, X., et al. (2011). Biological evaluation of a novel doxorubicin-peptide conjugate for targeted delivery to EGF receptor-overexpressing tumor cells. Molecular Pharmaceutics, 8, 375-386. https://doi.org/10.1021/mp100243j.

8. Ai, S., Jia, T., Ai, W., et al. (2013). Targeted delivery of doxorubicin through conjugation with EGF receptor-binding peptide overcomes drug resistance in human colon cancer cells. British Journal of Pharmacology, 168, 1719-1735. https://doi. org/10.1111/bph.12055

9. Alberici, L., Roth, L., Sugahara, K. N., et al. (2013). De Novo design of a tumor-penetrating peptide. Cancer Research, 73, 804812. https://doi.org/10.1158/0008-5472.CAN-12-1668.

10. Alfarouk, K. O., Stock, C.-M., Taylor, S., et al. (2015). Resistance to cancer chemotherapy: Failure in drug response from ADME to P-gp. Cancer Cell International, 15, 71. https://doi.org/10.1186/ s12935-015-0221-1.

11. Alibakhshi, A., Abarghooi Kahaki, F., Ahangarzadeh, S., et al. (2017). Targeted cancer therapy through antibody fragmentsdecorated nanomedicines. Journal of Controlled Release, 268, 323-334. https://doi.org/10.1016/J.JCONREL.2017.10.036.

12. Alitalo, K., \& Carmeliet, P. (2002). Molecular mechanisms of lymphangiogenesis in health and disease. Cancer Cell, 1, 219227. https://doi.org/10.1016/S1535-6108(02)00051-X.

13. Andrieu, J., Re, F., Russo, L., \& Nicotra, F. (2019). Phagedisplayed peptides targeting specific tissues and organs. Journal of Drug Targeting, 27, 555-565.

14. Arap, W., Pasqualini, R., \& Ruoslahti, E. (1998). Cancer treatment by targeted drug delivery to tumor vasculature in a mouse model. Science, 279, 377-380. https://doi.org/10.1126/ SCIENCE.279.5349.377.
15. Araste, F., Abnous, K., Hashemi, M., et al. (2018). Peptide-based targeted therapeutics: Focus on cancer treatment. Journal of Controlled Release, 292, 141-162.

16. Asciutto, E. K., Kopanchuk, S., Lepland, A., et al. (2019). Phagedisplay-derived peptide binds to human CD206 and modeling reveals a new binding site on the receptor. The Journal of Physical Chemistry. B, 123, 1973-1982. https://doi.org/10.1021/acs. jpcb.8b11876.

17. Augustin, H. G., \& Koh, G. Y. (2017). Organotypic vasculature: From descriptive heterogeneity to functional pathophysiology. Science, 357, 6353.

18. Azad, A. K., Rajaram, M. V. S., Metz, W. L., et al. (2015). $\gamma$-Tilmanocept, a new radiopharmaceutical tracer for cancer sentinel lymph nodes, binds to the mannose receptor (CD206). Journal of Immunology, 195, 2019-2029. https://doi.org/10.4049/ jimmunol.1402005.

19. Bábíčková, J., Tóthová, L., Boor, P., \& Celec, P. (2013). In vivo phage display - a discovery tool in molecular biomedicine. Biotechnology Advances, 31, 1247-1259. https://doi. org/10.1016/j.biotechadv.2013.04.004.

20. Barenholz, Y. (2012). Doxil® - The first FDA-approved nanodrug: Lessons learned. Journal of Controlled Release, 160, 117-134.

21. Barrow, P. A., \& Soothill, J. S. (1997). Bacteriophage therapy and prophylaxis: Rediscovery and renewed assessment of potential. Trends in Microbiology, 5, 268-271.

22. Baudino, T. A. (2015). Targeted cancer therapy: The next generation of cancer treatment. Current Drug Discovery Technologies, 12, 3-20. https://doi.org/10.2174/15701638126 66150602144310.

23. Beck, A., Goetsch, L., Dumontet, C., \& Corvaïa, N. (2017). Strategies and challenges for the next generation of antibody-drug conjugates. Nature Reviews. Drug Discovery, 16(5), 315-337. https://doi.org/10.1038/nrd.2016.268.

24. Becker, J. M., \& Naider, F. (2015). Cross-linking strategies to study peptide ligand-receptor interactions. In Methods in enzymology (pp. 527-547). New York: Academic Press.

25. Benezra, M., Penate-Medina, O., Zanzonico, P. B., et al. (2011). Multimodal silica nanoparticles are effective cancer-targeted probes in a model of human melanoma. The Journal of Clinical Investigation, 121, 2768-2780. https://doi.org/10.1172/JCI45600.

26. Berger, C. M., Gaume, X., \& Bouvet, P. (2015). The roles of nucleolin subcellular localization in cancer. Biochimie, 113, 78-85.

27. Bhagwat, S. V., Lahdenranta, J., Giordano, R., et al. (2001). CD13/ APN is activated by angiogenic signals and is essential for capillary tube formation. Blood, 97, 652-659. https://doi.org/10.1182/ blood.V97.3.652.

28. Bobo, D., Robinson, K. J., Islam, J., et al. (2016). Nanoparticlebased medicines: A review of FDA-approved materials and clinical trials to date. Pharmaceutical Research, 33, 2373-2387.

29. Braun, G. B., Sugahara, K. N., Yu, O. M., et al. (2016), Urokinase-controlled tumor penetrating peptide. Journal of Controlled Release, 232, 188-195. https://doi.org/10.1016/j. jconrel.2016.04.027.

30. Bray, F., Ferlay, J., Soerjomataram, I., et al. (2018). Global cancer statistics 2018: GLOBOCAN estimates of incidence and mortality worldwide for 36 cancers in 185 countries. CA: a Cancer Journal for Clinicians, 68, 394-424. https://doi.org/10.3322/caac.21492.

31. Burg, M. A., Pasqualini, R., Arap, W., et al. (1999). NG2 proteoglycan-binding peptides target tumor neovasculature. Cancer Research, 59, 2869-2874.

32. Burugu, S., Dancsok, A. R., \& Nielsen TO. (2018). Emerging targets in cancer immunotherapy. Seminars in Cancer Biology, 52, 39-52. https://doi.org/10.1016/J.SEMCANCER.2017.10.001.

33. Cai, J., Kehoe, O., Smith, G. M., et al. (2008). The angiopoietin/Tie-2 system regulates pericyte survival and recruitment in 
diabetic retinopathy. Investigative Ophthalmology and Visual Science, 49, 2163-2171. https://doi.org/10.1167/iovs.07-1206.

34. Carnemolla, B., Castellani, P., Ponassi, M., et al. (1999). Identification of a glioblastoma-associated tenascin-C isoform by a high affinity recombinant antibody. The American Journal of Pathology, 154, 1345-1352. https://doi.org/10.1016/ S0002-9440(10)65388-6.

35. Carter, P. J., \& Lazar, G. A. (2018). Next generation antibody drugs: Pursuit of the "high-hanging fruit". Nature Reviews. Drug Discovery, 17, 197-223.

36. Chabner, B. A., \& Roberts, T. G. (2005). Chemotherapy and the war on cancer. Nature Reviews. Cancer, 5, 65-72.

37. Chang, L., Chen, Y. J., Fan, C. Y., et al. (2017). Identification of siglec ligands using a proximity labeling method. Journal of Proteome Research, 16, 3929-3941. https://doi.org/10.1021/acs. jproteome.7b00625.

38. Chanmee, T., Ontong, P., Konno, K., \& Itano, N. (2014). Tumorassociated macrophages as major players in the tumor microenvironment. Cancers (Basel), 6, 1670-1690.

39. Chen, Y., Shen, Y., Guo, X., et al. (2006). Transdermal protein delivery by a coadministered peptide identified via phage display. Nature Biotechnology, 24, 455-460. https://doi.org/10.1038/ nbt1193.

40. Cheng, H., Chi, C., Shang, W., et al. (2017). Precise integrintargeting near-infrared imaging-guided surgical method increases surgical qualification of peritoneal carcinomatosis from gastric cancer in mice. Oncotarget, 8(4), 6258. https://doi.org/10.18632/ oncotarget.14058.

41. Christian, S., Pilch, J., Akerman, M. E., et al. (2003). Nucleolin expressed at the cell surface is a marker of endothelial cells in angiogenic blood vessels. The Journal of Cell Biology, 163, 871878. https://doi.org/10.1083/jcb.200304132.

42. Cieslewicz, M., Tang, J., Yu, J. L., et al. (2013). Targeted delivery of proapoptotic peptides to tumor-associated macrophages improves survival. Proceedings of the National Academy of Sciences, 110, 15919-15924. https://doi.org/10.1073/pnas.1312197110.

43. Clackson, T., Hoogenboom, H. R., Griffiths, A. D., \& Winter, G. (1991). Making antibody fragments using phage display libraries. Nature, 352, 624-628. https://doi.org/10.1038/352624a0.

44. Curnis, F., Arrigoni, G., Sacchi, A., et al. (2002). Differential binding of drugs containing the NGR motif to CD13 isoforms in tumor vessels, epithelia, and myeloid cells. Cancer Research, 62, 867-874.

45. Gerber, D. E. (2008). Targeted therapies: A new generation of cancer treatments. American Family Physician, 77, 311-319.

46. Danhier, F. (2016). To exploit the tumor microenvironment: Since the EPR effect fails in the clinic, what is the future of nanomedicine? Journal of Controlled Release, 244, 108-121.

47. Daniels, D. A., Chen, H., Hicke, B. J., et al. (2003). A tenascin-C aptamer identified by tumor cell SELEX: Systematic evolution of ligands by exponential enrichment. Proceedings of the National Academy of Sciences of the United States of America, 100, 1541615421. https://doi.org/10.1073/pnas.2136683100.

48. Dawar, S., Singh, N., Kanwar, R. K., et al. (2013). Multifunctional and multitargeted nanoparticles for drug delivery to overcome barriers of drug resistance in human cancers. Drug Discovery Today, 18, 1292-1300.

49. Dechantsreiter, M. A., Planker, E., Mathä, B., et al. (1999). $\mathrm{N}$-methylated cyclic RGD peptides as highly active and selective $\alpha(\mathrm{v}) \beta 3$ integrin antagonists. Journal of Medicinal Chemistry, 42, 3033-3040. https://doi.org/10.1021/jm970832g.

50. Deng, X., Wang, L., You, X., et al. (2017). Advances in the T7 phage display system (Review). Molecular Medicine Reports, 17, 714-720. https://doi.org/10.3892/mmr.2017.7994.

51. Desgrosellier, J. S., \& Cheresh, D. A. (2010). Integrins in cancer: Biological implications and therapeutic opportunities. Nature Reviews. Cancer, 10, 9-22. https://doi.org/10.1038/nrc2748.
52. Destouches, D., El Khoury, D., Hamma-Kourbali, Y., et al. (2008). Suppression of tumor growth and angiogenesis by a specific antagonist of the cell-surface expressed nucleolin. PLoS One, 3, e2518. https://doi.org/10.1371/journal.pone.0002518.

53. Drecoll, E., Gaertner, F. C., Miederer, M., et al. (2009). Treatment of peritoneal carcinomatosis by targeted delivery of the radiolabeled tumor homing peptide 213Bi-DTPA-[F3]2 into the nucleus of tumor cells. PLoS One, 4, e5715. https://doi.org/10.1371/journal.pone.0005715.

54. Edwards, D. R., Handsley, M. M., \& Pennington, C. J. (2009). The ADAM metalloproteinases. Molecular Aspects of Medicine, 29, 258-289.

55. El Khoury, D., Destouches, D., Lengagne, R., et al. (2010). Targeting surface nucleolin with a multivalent pseudopeptide delays development of spontaneous melanoma in RET transgenic mice. BMC Cancer, 10, 325. https://doi. org/10.1186/1471-2407-10-325.

56. Ellerby, H. M., Arap, W., Ellerby, L. M., et al. (1999). Anti-cancer activity of targeted pro-apoptotic peptides. Nature Medicine, 5, 1032-1038. https://doi.org/10.1038/12469.

57. Field, L. D., Delehanty, J. B., Chen, Y., \& Medintz, I. L. (2015). Peptides for specifically targeting nanoparticles to cellular organelles: Quo vadis ? Accounts of Chemical Research, 48, 13801390. https://doi.org/10.1021/ar500449v.

58. Fosgerau, K., \& Hoffmann, T. (2015). Peptide therapeutics: Current status and future directions. Drug Discovery Today, 20, 122-128. https://doi.org/10.1016/j.drudis.2014.10.003.

59. Frantz, C., Stewart, K. M., \& Weaver, V. M. (2010a). The extracellular matrix at a glance. Journal of Cell Science, 123, 4195-4200.

60. Garde, S. V., Forté, A. J., Ge, M., et al. (2007). Binding and internalization of NGR-peptide-targeted liposomal doxorubicin (TVT-DOX) in CD13-expressing cells and its antitumor effects. Anti-Cancer Drugs, 18, 1189-1200. https://doi.org/10.1097/ CAD.0b013e3282a213ce.

61. Geretti, E., \& Klagsbrun, M. (2007). Neuropilins: Novel targets for anti-angiogenesis therapies. Cell Adhesion \& Migration, 1, $56-61$.

62. Gregorc, V., Gaafar, R. M., Favaretto, A., et al. (2018). NGRhTNF in combination with best investigator choice in previously treated malignant pleural mesothelioma (NGR015): A randomised, double-blind, placebo-controlled phase 3 trial. The Lancet Oncology, 19, 799-811. https://doi.org/10.1016/ S1470-2045(18)30193-1.

63. Gregorc, V., Santoro, A., Bennicelli, E., et al. (2009). Phase Ib study of NGR-hTNF, a selective vascular targeting agent, administered at low doses in combination with doxorubicin to patients with advanced solid tumours. British Journal of Cancer, 101, 219-224. https://doi.org/10.1038/sj.bjc.6605162.

64. Gurunathan, S., Kang, M. H., Qasim, M., \& Kim, J. H. (2018). Nanoparticle-mediated combination therapy: Two-in-one approach for cancer. International Journal of Molecular Sciences, 19(10), 3264.

65. Guzman-Rojas, L., Rangel, R., Salameh, A., et al. (2012). Cooperative effects of aminopeptidase N (CD13) expressed by nonmalignant and cancer cells within the tumor microenvironment. Proceedings of the National Academy of Sciences of the United States of America, 109, 1637-1642. https://doi. org/10.1073/pnas.1120790109.

66. Hambley, T. W. (2009). Is anticancer drug development heading in the right direction? Cancer Research, 69, 1259-1261.

67. Han, Z., Zhou, Z., Shi, X., et al. (2015). EDB fibronectin specific peptide for prostate cancer targeting. Bioconjugate Chemistry, 26(5), 830-836. https://doi.org/10.1021/acs. bioconjchem.5b00178.

68. Hanahan, D., \& Folkman, J. (1996). Patterns and emerging mechanisms of the angiogenic switch during tumorigenesis. Cell, 86, $353-364$. 
69. Hanahan, D., \& Weinberg, R. A. (2011). Hallmarks of cancer: The next generation. Cell, 144, 646-674.

70. Hanold, L. E., Oruganty, K., Ton, N. T., et al. (2015). Inhibiting EGFR dimerization using triazolyl-bridged dimerization arm mimics. PLoS One, 10, e0118796. https://doi.org/10.1371/journal. pone. 0118796 .

71. Harris, L., Batist, G., Belt, R., et al. (2002). Liposomeencapsulated doxorubicin compared with conventional doxorubicin in a randomized multicenter trial as first-line therapy of metastatic breast carcinoma. Cancer, 94, 25-36. https://doi. org/10.1002/cncr.10201.

72. Heldin, C. H., Rubin, K., Pietras, K., \& Östman, A. (2004). High interstitial fluid pressure - An obstacle in cancer therapy. Nature Reviews. Cancer, 4, 806-813.

73. Hoffman, J. A., Giraudo, E., Singh, M., et al. (2003). Progressive vascular changes in a transgenic mouse model of squamous cell carcinoma. Cancer Cell, 4, 383-391. https://doi.org/10.1016/ S1535-6108(03)00273-3.

74. Hossein-Nejad-Ariani, H., Althagafi, E., \& Kaur, K. (2019). Small peptide ligands for targeting EGFR in triple negative breast cancer cells. Scientific Reports, 9, 2723. https://doi.org/10.1038/ s41598-019-38574-y.

75. Kaufman, H. L., \& Scott Wadler, K. A. (2008). Molecular targeting in oncology. Totowa, NJ: Humana Press.

76. Hu, Q., Gu, G., Liu, Z., et al. (2013). F3 peptide-functionalized PEG-PLA nanoparticles co-administrated with tLyp-1 peptide for anti-glioma drug delivery. Biomaterials, 34, 1135-1145. https:// doi.org/10.1016/j.biomaterials.2012.10.048.

77. Huang, C.-Y., Ju, D.-T., Chang, C.-F., et al. (2017). A review on the effects of current chemotherapy drugs and natural agents in treating non-small cell lung cancer. Biomedicine, 7, 23. https:// doi.org/10.1051/bmden/2017070423.

78. Hughes, R., Qian, B.-Z., Rowan, C., et al. (2015). Perivascular M2 macrophages stimulate tumor relapse after chemotherapy. Cancer Research, 75, 3479-3491. https://doi.org/10.1158/0008-5472. CAN-14-3587.

79. Hung, V., Udeshi, N. D., Lam, S. S., et al. (2016). Spatially resolved proteomic mapping in living cells with the engineered peroxidase APEX2. Nature Protocols, 11, 456-475. https://doi. org/10.1038/nprot.2016.018.

80. Hunt, H., Simón-Gracia, L., Tobi, A., et al. (2017). Targeting of p32 in peritoneal carcinomatosis with intraperitoneal linTT1 peptide-guided pro-apoptotic nanoparticles. Journal of Controlled Release, 260, 142-153. https://doi.org/10.1016/j. jconrel.2017.06.005.

81. Hussain, S., Rodriguez-Fernandez, M., Braun, G. B., et al. (2014). Quantity and accessibility for specific targeting of receptors in tumours. Scientific Reports, 4, 5232. https://doi.org/10.1038/ srep05232.

82. Ikemoto, H., Lingasamy, P., Willmore, A. M. A., et al. (2017). Hyaluronan-binding peptide for targeting peritoneal carcinomatosis. Tumor Biology, 39, 1010428317701628. https://doi. org/10.1177/1010428317701628.

83. Ivanenkov, V. V., Felici, F., \& Menon, A. G. (1999). Targeted delivery of multivalent phage display vectors into mammalian cells. Biochimica et Biophysica Acta (BBA)-Molecular Cell Research, 1448, 463-472. https://doi.org/10.1016/ S0167-4889(98)00163-3.

84. Jain, R. K. (2005). Normalization of tumor vasculature: An emerging concept in antiangiogenic therapy. Science, 307, 58-62.

85. Jain, R. K., \& Baxter, L. T. (1988). Mechanisms of heterogeneous distribution of monoclonal antibodies and other macromolecules in tumors: Significance of elevated interstitial pressure. Cancer Research, 48, 7022-7032.

86. Järveläinen, H. (2009). Extracellular matrix molecules: Potential targets in pharmacotherapy. Pharmacological Reviews, 61, 198223. https://doi.org/10.1124/pr.109.001289.provided.
87. Järveläinen, H., Sainio, A., Koulu, M., et al. (2009). Extracellular matrix molecules: Potential targets in pharmacotherapy. Pharmacological Reviews, 61, 198-223.

88. Jaynes, J. M., Sable, R., Ronzetti, M., et al. (2020). Mannose receptor (CD206) activation in tumor-associated macrophages enhances adaptive and innate antitumor immune responses. Science Translational Medicine, 12, eaax6337. https://doi. org/10.1126/scitranslmed.aax6337.

89. Jiang, D., Xu, M., Pei, Y., et al. (2019). Core-matched nanoassemblies for targeted co-delivery of chemotherapy and photosensitizer to treat drug-resistant cancer. Acta Biomaterialia, 88, 406-421. https://doi.org/10.1016/j.actbio.2019.02.009.

90. Jiang, K., Song, X., Yang, L., et al. (2018). Enhanced antitumor and anti-metastasis efficacy against aggressive breast cancer with a fibronectin-targeting liposomal doxorubicin. Journal of Controlled Release, 271, 21-30. https://doi.org/10.1016/j. jconrel.2017.12.026.

91. Jin, Z.-H., Josserand, V., Razkin, J., et al. (2006). Noninvasive optical imaging of ovarian metastases using Cy5-labeled RAFTc(-RGDfK-)4. Molecular Imaging, 5, 188-197.

92. Johansson, A., Hamzah, J., \& Ganss, R. (2014). License for destruction: Tumor-specific cytokine targeting. Trends in Molecular Medicine, 20, 16-24.

93. Joyce, J. A., Laakkonen, P., Bernasconi, M., et al. (2003). Stagespecific vascular markers revealed by phage display in a mouse model of pancreatic islet tumorigenesis. Cancer Cell, 4, 393-403. https://doi.org/10.1016/S1535-6108(03)00271-X.

94. Kapoor, P., Singh, H., Gautam, A., et al. (2012). Tumorhope: A database of tumor homing peptides. PLoS One, 7, e35187. https:// doi.org/10.1371/journal.pone.0035187.

95. Karamchand, L., Kim, G., Wang, S., et al. (2013). Modulation of hydrogel nanoparticle intracellular trafficking by multivalent surface engineering with tumor targeting peptide. Nanoscale, 5, 10327-10344. https://doi.org/10.1039/c3nr00908d.

96. Keat, N., Kenny, J., Chen, K., et al. (2018). A microdose PET study of the safety, immunogenicity, biodistribution, and radiation dosimetry of 18 F-FB-A20FMDV2 for imaging the integrin $\alpha \mathrm{v} \beta$ 6. Journal of Nuclear Medicine Technology, 46, 136-143. https:// doi.org/10.2967/jnmt.117.203547.

97. Kim, D. I., Jensen, S. C., Noble, K. A., et al. (2016). An improved smaller biotin ligase for BioID proximity labeling. Molecular Biology of the Cell, 27, 1188-1196. https://doi.org/10.1091/mbc. E15-12-0844.

98. Kim, M. Y., Kim, O. R., Choi, Y. S., et al. (2012). Selection and characterization of tenascin $\mathrm{C}$ targeting peptide. Molecules and Cells, 33, 71-77. https://doi.org/10.1007/s10059-012-2214-4.

99. Kimura, R. H., Teed, R., Hackel, B. J., et al. (2012). Pharmacokinetically stabilized cystine knot peptides that bind Alpha-v-Beta-6 integrin with single-digit nanomolar affinities for detection of pancreatic cancer. Clinical Cancer Research, 18, 839-849. https://doi.org/10.1158/1078-0432.CCR-11-1116.

100. Kirpotin, D. B., Drummond, D. C., Shao, Y., et al. (2006). Antibody targeting of long-circulating lipidic nanoparticles does not increase tumor localization but does increase internalization in animal models. Cancer Research, 66, 6732-6740. https://doi. org/10.1158/0008-5472.CAN-05-4199.

101. Koivunen, E., Gay, D. A., \& Ruoslahti, E. (1993). Selection of peptides binding to the alpha 5 beta 1 integrin from phage display library. The Journal of Biological Chemistry, 268, 20205-20210.

102. Kolvunen, E., Wang, B., \& Ruoslahti, E. (1995). Phage libraries displaying cyclic peptides with different ring sizes: Ligand specificities of the rgd-directed integrine. Bio/Technology, 13, 265-270. https://doi.org/10.1038/nbt0395-265.

103. Komoriya, A., Hortsch, M., \& Meyers, C. (1984). Biologically active synthetic fragments of epidermal growth factor: Localization of a major receptor-binding region. Isotopenpraxis, 20, 1351-1355. https://doi.org/10.1073/pnas.81.5.1351. 
104. Kruger, G., Bandyopadhyay, A., \& Gao, J. (2017). Cancertargeting peptides. In Advances in the discovery and development of peptide therapeutics (pp. 70-82). Singapore: Springer International Publishing.

105. Kumra, H., \& Reinhardt, D. P. (2016). Fibronectin-targeted drug delivery in cancer. Advanced Drug Delivery Reviews, 97, 101110. https://doi.org/10.1016/j.addr.2015.11.014.

106. Kuncic, Z. (2015a). Cancer nanomedicine: Challenges and opportunities. Medical Journal of Australia, 203, 204-205.e1.

107. Laakkonen, P., Akerman, M. E., Biliran, H., et al. (2004a). Antitumor activity of a homing peptide that targets tumor lymphatics and tumor cells. Proceedings of the National Academy of Sciences, 101, 9381-9386. https://doi.org/10.1073/ pnas.0403317101.

108. Laakkonen, P., Akerman, M. E., Biliran, H., et al. (2004b). Antitumor activity of a homing peptide that targets tumor lymphatics and tumor cells. Proceedings of the National Academy of Sciences of the United States of America, 101, 9381. https://doi. org/10.1073/pnas.0403317101.

109. Laakkonen, P., Porkka, K., Hoffman, J. A., \& Ruoslahti, E. (2002a). A tumor-homing peptide with a targeting specificity related to lymphatic vessels. Nature Medicine, 8, 751-755. https:// doi.org/10.1038/nm720.

110. Laakkonen, P., Zhang, L., \& Ruoslahti, E. (2008). Peptide targeting of tumor lymph vessels. In Annals of the New York academy of sciences (Vol. 1131, pp. 37-43). John Wiley \& Sons, Ltd (10.1111). https://doi.org/10.1196/annals.1413.003.

111. Lambert, J. M., \& Morris, C. Q. (2017). Antibody-drug conjugates (ADCs) for personalized treatment of solid tumors: A review. Advances in Therapy, 34(5), 1015-1035. https://doi.org/10.1007/ s12325-017-0519-6.

112. Larocca, D., Kassner, P. D., Witte, A., et al. (1999). Gene transfer to mammalian cells using genetically targeted filamentous bacteriophage. The FASEB Journal, 13, 727-734. https://doi. org/10.1096/fasebj.13.6.727.

113. Lau, J. L., \& Dunn, M. K. (2018). Therapeutic peptides: Historical perspectives, current development trends, and future directions. Bioorganic \& Medicinal Chemistry, 26, 2700-2707. https://doi. org/10.1016/j.bmc.2017.06.052.

114. Le Joncour, V., \& Laakkonen, P. (2018). Seek \& Destroy, use of targeting peptides for cancer detection and drug delivery. Bioorganic \& Medicinal Chemistry, 26, 2797-2806. https://doi. org/10.1016/j.bmc.2017.08.052.

115. Lingasamy, P., Tobi, A., Haugas, M., et al. (2019). Bi-specific tenascin-C and fibronectin targeted peptide for solid tumor delivery. Biomaterials, 219, 119373. https://doi.org/10.1016/j. biomaterials.2019.119373.

116. Liu, N., Lapcevich, R. K., Underhill, C. B., et al. (2001). Metastatin: A hyaluronan-binding complex from cartilage that inhibits tumor growth. Cancer Research, 61, 1022-1028.

117. Liu, R., Li, X., Xiao, W., \& Lam, K. S. (2017). Tumor-targeting peptides from combinatorial libraries. Advanced Drug Delivery Reviews, 110-111, 13-37.

118. Lorusso, D., Scambia, G., Amadio, G., et al. (2012). Phase II study of NGR-hTNF in combination with doxorubicin in relapsed ovarian cancer patients. British Journal of Cancer, 107, 37-42. https://doi.org/10.1038/bjc.2012.233.

119. Lu, L., Qi, H., Zhu, J., et al. (2017). Vascular-homing peptides for cancer therapy. Biomedicine \& Pharmacotherapy, 92, 187-195. https://doi.org/10.1016/j.biopha.2017.05.054.

120. Maeda, H. (2010). Tumor-selective delivery of macromolecular drugs via the EPR effect: Background and future prospects. Bioconjugate Chemistry, 21(5), 797-802. https://doi.org/10.1021/ bc $100070 \mathrm{~g}$.

121. Maeda, H. (2012). Macromolecular therapeutics in cancer treatment: The EPR effect and beyond. Journal of Controlled Release, $164,138-144$.
122. Maeda, H., Nakamura, H., \& Fang, J. (2013). The EPR effect for macromolecular drug delivery to solid tumors: Improvement of tumor uptake, lowering of systemic toxicity, and distinct tumor imaging in vivo. Advanced Drug Delivery Reviews, 65, 71-79.

123. Maeda, H., Wu, J., Sawa, T., et al. (2000). Tumor vascular permeability and the EPR effect in macromolecular therapeutics: A review. Journal of Controlled Release, 65, 271-284. https://doi. org/10.1016/S0168-3659(99)00248-5.

124. Mahmood, N., Mihalcioiu, C., \& Rabbani, S. A. (2018). Multifaceted role of the urokinase-type plasminogen activator (uPA) and its receptor (uPAR): Diagnostic, prognostic, and therapeutic applications. Frontiers in Oncology, 8, 24.

125. Marelli, U. K., Rechenmacher, F., Sobahi, T. R. A., et al. (2013). Tumor targeting via integrin ligands. Frontiers in Oncology, 3, 222. https://doi.org/10.3389/fonc.2013.00222.

126. Matochko, W. L., \& Derda, R. (2015). Next-generation sequencing of phage-displayed peptide libraries. Methods in Molecular Biology, 1248, 249-266. https://doi. org/10.1007/978-1-4939-2020-4_17.

127. McFedries, A., Schwaid, A., \& Saghatelian, A. (2013). Methods for the elucidation of protein-small molecule interactions. Chemistry \& Biology, 20, 667-673.

128. Miao, H. Q., \& Klagsbrun, M. (2000). Neuropilin is a mediator of angiogenesis. Cancer Metastasis Reviews, 19, 29-37.

129. Mitri, Z., Constantine, T., \& O'Regan, R. (2012). The HER2 receptor in breast Cancer: Pathophysiology, clinical use, and new advances in therapy. Chemotherapy Research and Practice, 2012, 1-7. https://doi.org/10.1155/2012/743193.

130. Nagy, J. A., Chang, S. H., Dvorak, A. M., \& Dvorak, H. F. (2009). Why are tumour blood vessels abnormal and why is it important to know? British Journal of Cancer, 100, 865-869.

131. Neri, D., \& Bicknell, R. (2005). Tumour vascular targeting. Nature Reviews. Cancer, 5, 436-446. https://doi.org/10.1038/nrc1627.

132. Nilsson, F., Kosmehl, H., Zardi, L., \& Neri, D. (2001). Targeted delivery of tissue factor to the ED-B domain of fibronectin, a marker of angiogenesis, mediates the infarction of solid tumors in mice. Cancer Research, 61, 711-716.

133. Nishida, N., Yano, H., Nishida, T., et al. (2006). Angiogenesis in cancer. Vascular Health and Risk Management, 2, 213-219.

134. Nisole, S., Krust, B., Callebaut, C., et al. (1999). The anti-HIV pseudopeptide HB-19 forms a complex with the cell-surfaceexpressed nucleolin independent of heparan sulfate proteoglycans. The Journal of Biological Chemistry, 274, 27875-27884. https://doi.org/10.1074/jbc.274.39.27875.

135. Nolan, D. J., Ginsberg, M., Israely, E., et al. (2013). Molecular signatures of tissue-specific microvascular endothelial cell heterogeneity in organ maintenance and regeneration. Developmental Cell, 26, 204-219. https://doi.org/10.1016/j. devcel.2013.06.017.

136. Noy, R., \& Pollard, J. W. (2014). Tumor-associated macrophages: From mechanisms to therapy. Immunity, 41, 49-61.

137. Paasonen, L., Sharma, S., Braun, G. B., et al. (2016). New p32/ gC1qR ligands for targeted tumor drug delivery. Chembiochem, 17, 570-575. https://doi.org/10.1002/cbic.201500564.

138. Pagé, M. (2002). Tumor targeting in cancer therapy. Totowa: Humana Press.

139. Pang, H.-B., Braun, G. B., Friman, T., et al. (2014). An endocytosis pathway initiated through neuropilin-1 and regulated by nutrient availability. Nature Communications, 5, 4904. https://doi. org/10.1038/ncomms5904.

140. Park, J., Kim, S., Saw, P. E., et al. (2012). Fibronectin extra domain B-specific aptide conjugated nanoparticles for targeted cancer imaging. Journal of Controlled Release, 163, 111-118. https://doi.org/10.1016/j.jconrel.2012.08.029.

141. Park, J. W., Hong, K., Kirpotin, D. B., et al. (2002). Anti-HER2 immunoliposomes: Enhanced efficacy attributable to targeted delivery. Clinical Cancer Research, 8, 1172-1181. 
142. Pasqualini, R., Koivunen, E., Kain, R., et al. (2000). Aminopeptidase $\mathrm{N}$ is a receptor for tumor-homing peptides and a target for inhibiting angiogenesis. Cancer Research, 60, 722-727.

143. Pasqualini, R., \& Ruoslahti, E. (1996). Organ targeting in vivo using phage display peptide libraries. Nature, 380, 364-366. https://doi.org/10.1038/380364a0.

144. Pastorino, F., Brignole, C., Di Paolo, D., et al. (2006). Targeting liposomal chemotherapy via both tumor cell-specific and tumor vasculature-specific ligands potentiates therapeutic efficacy. Cancer Research, 66, 10073-10082. https://doi.org/10.1158/00085472.CAN-06-2117.

145. Pastorino, F., Brignole, C., Marimpietri, D., et al. (2003). Vascular damage and anti-angiogenic effects of tumor vessel-targeted liposomal chemotherapy. Cancer Research, 63, 7400-7409.

146. Penchala, S. C., Miller, M. R., Pal, A., et al. (2015). A biomimetic approach for enhancing the in vivo half-life of peptides. Nature Chemical Biology, 11, 793-798. https://doi.org/10.1038/ nchembio.1907.

147. Persson, B. E., Kold Olesen, T., \& Jensen, J. K. (2009). Degarelix: A new approach for the treatment of prostate cancer. Neuroendocrinology, 90, 235-244. https://doi. org/10.1159/000228832.

148. Pierschbacher, M. D., \& Ruoslahti, E. (1984). Cell attachment activity of fibronectin can be duplicated by small synthetic fragments of the molecule. Nature, 309, 30-33. https://doi. org/10.1038/309030a0.

149. Pierschbacher, M. D., Ruoslahti, E., Sundelin, J., et al. (1982). The cell attachment domain of fibronectin. Determination of the primary structure. Journal of Biological Chemistry, 257, 9593-9597.

150. Pilch, J., Brown, D. M., Komatsu, M., et al. (2006). Peptides selected for binding to clotted plasma accumulate in tumor stroma and wounds. Proceedings of the National Academy of Sciences of the United States of America, 103, 2800-2804. https://doi. org/10.1073/pnas.0511219103.

151. Pollard, J. W. (2004). Tumour-educated macrophages promote tumour progression and metastasis. Nature Reviews. Cancer, 4, 71-78. https://doi.org/10.1038/nrc1256.

152. Porkka, K., Laakkonen, P., Hoffman, J. A., et al. (2002). A fragment of the HMGN2 protein homes to the nuclei of tumor cells and tumor endothelial cells in vivo. Proceedings of the National Academy of Sciences, 99, 7444-7449. https://doi.org/10.1073/ pnas.062189599.

153. Prabhakar, U., Maeda, H., Jain, R. K., et al. (2013). Challenges and key considerations of the enhanced permeability and retention effect for nanomedicine drug delivery in oncology. Cancer Research, 73, 2412-2417. https://doi.org/10.1158/0008-5472. CAN-12-4561.

154. Prickett, W. M., Van Rite, B. D., Resasco, D. E., \& Harrison, R. G. (2011). Vascular targeted single-walled carbon nanotubes for near-infrared light therapy of cancer. Nanotechnology, 22, 455101. https://doi.org/10.1088/0957-4484/22/45/455101.

155. Pytela, R., Pierschbacher, M. D., Ginsberg, M. H., et al. (1986). Platelet membrane glycoprotein IIb/IIIa: Member of a family of Arg-Gly-Asp-specific adhesion receptors. Science, 231, 15591562. https://doi.org/10.1126/science.2420006.

156. Pytela, R., Pierschbacher, M. D., \& Ruoslahti, E. (1985). Identification and isolation of a $140 \mathrm{kd}$ cell surface glycoprotein with properties expected of a fibronectin receptor. Cell, 40, 191198. https://doi.org/10.1016/0092-8674(85)90322-8.

157. Qin, M., Zong, H., \& Kopelman, R. (2014). Click conjugation of peptide to hydrogel nanoparticles for tumor-targeted drug delivery. Biomacromolecules, 15, 3728-3734. https://doi.org/10.1021/ bm501028c.

158. Rajabi, M., \& Mousa, S. (2017). The role of angiogenesis in cancer treatment. Biomedicine, 5, 34. https://doi.org/10.3390/ biomedicines 5020034 .
159. Ramsay, R. R., Popovic-Nikolic, M. R., Nikolic, K., et al. (2018). A perspective on multi-target drug discovery and design for complex diseases. Clinical and Translational Medicine, 7(1), 3. https://doi.org/10.1186/s40169-017-0181-2.

160. Raucher, D. (2019). Tumor targeting peptides: Novel therapeutic strategies in glioblastoma. Current Opinion in Pharmacology, 47, $14-19$.

161. Rosenblum, D., Joshi, N., Tao, W., et al. (2018a). Progress and challenges towards targeted delivery of cancer therapeutics. Nature Communications, 9, 1-12.

162. Rosenblum, D., Joshi, N., Tao, W., et al. (2018b). Progress and challenges towards targeted delivery of cancer therapeutics. Nature Communications, 9, 1410.

163. Roth, L., Agemy, L., Kotamraju, V. R., et al. (2012). Transtumoral targeting enabled by a novel neuropilin-binding peptide. Oncogene, 31, 3754-3763. https://doi.org/10.1038/onc.2011.537.

164. Ruoslahti, E. (2004). Vascular zip codes in angiogenesis and metastasis. Biochemical Society Transactions, 32, 397-402.

165. Ruoslahti, E. (2012). Peptides as targeting elements and tissue penetration devices for nanoparticles. Advanced Materials, 24, 3747-3756.

166. Ruoslahti, E. (2000). Targeting tumor vasculature with homing peptides from phage display. Cancer Biology, 10, 435-442. https://doi.org/10.1006/scbi.2000.0334.

167. Ruoslahti, E. (2017). Tumor penetrating peptides for improved drug delivery. Advanced Drug Delivery Reviews, 110-111, 3-12. https://doi.org/10.1016/j.addr.2016.03.008.

168. Ruoslahti, E. (2002). Specialization of tumour vasculature. Nature Reviews. Cancer, 2, 83-90. https://doi.org/10.1038/nrc724.

169. Ruoslahti, E., Bhatia, S. N., \& Sailor, M. J. (2010). Targeting of drugs and nanoparticles to tumors. The Journal of Cell Biology, $188,759-768$.

170. Ruoslahti, E., \& Pierschbacher, M. D. (1987). New perspectives in cell adhesion: RGD and integrins. Science, 238, 491-497. https:// doi.org/10.1126/science.2821619.

171. Ryan, J. M., Wasser, J. S., Adler, A. J., \& Vella, A. T. (2016). Enhancing the safety of antibody-based immunomodulatory cancer therapy without compromising therapeutic benefit: Can we have our cake and eat it too? Expert Opinion on Biological Therapy, 16, 655-674. https://doi.org/10.1517/14712598.2016.11 52256.

172. Ebbinghaus, S., Hussain, M., Tannir, N., Gordon, M., et al. (2007). Phase 2 study of ABT-510 in patients with previously untreated advanced renal cell carcinoma. In Clinical cancer research (pp. 6689-6695). Philadelphia: American Association for Cancer Research.

173. Säälik, P., Lingasamy, P., Toome, K., et al. (2019). Peptideguided nanoparticles for glioblastoma targeting. Journal of Controlled Release, 308, 109-118. https://doi.org/10.1016/j. jconrel.2019.06.018.

174. Sawa-Wejksza, K., \& Kandefer-Szerszeń, M. (2018). Tumorassociated macrophages as target for antitumor therapy. Archivum Immunologiae et Therapiae Experimentalis (Warsz), 66, 97-111.

175. Scodeller, P., Simón-Gracia, L., Kopanchuk, S., et al. (2017). Precision targeting of tumor macrophages with a CD206 binding peptide. Scientific Reports, 7, 14655. https://doi.org/10.1038/ s41598-017-14709-x.

176. Sharkey, R. M., \& Goldenberg, D. M. (2005). Perspectives on cancer therapy with radiolabeled monoclonal antibodies. Journal of Nuclear Medicine, 46(Suppl 1), 115S-127S.

177. Sharma, R., Kallur, K. G., Ryu, J. S., et al. (2015). Multicenter reproducibility of $18 \mathrm{~F}$-Fluciclatide PET imaging in subjects with solid tumors. Journal of Nuclear Medicine, 56, 1855-1861. https://doi.org/10.2967/jnumed.115.158253.

178. Sharma, S., Kotamraju, V. R., Mölder, T., et al. (2017). Tumorpenetrating nanosystem strongly suppresses breast tumor growth. 
Nano Letters, 17, 1356-1364. https://doi.org/10.1021/acs. nanolett.6b03815.

179. Shi, Q., Zhang, Y., Liu, S., et al. (2018). Specific tissue factor delivery using a tumor-homing peptide for inducing tumor infarction. Biochemical Pharmacology, 156, 501-510. https://doi. org/10.1016/j.bcp.2018.09.020.

180. Silacci, M., Brack, S. S., Späth, N., et al. (2006). Human monoclonal antibodies to domain $\mathrm{C}$ of tenascin-C selectively target solid tumors in vivo. Protein Engineering, Design \& Selection, 19, 471-478. https://doi.org/10.1093/protein/gzl033.

181. Simberg, D., Duza, T., Park, J. H., et al. (2007). Biomimetic amplification of nanoparticle homing to tumors. Proceedings of the National Academy of Sciences of the United States of America, 104, 932-936. https://doi.org/10.1073/pnas.0610298104.

182. Simón-Gracia, L., Hunt, H., \& Teesalu, T. (2018). Peritoneal carcinomatosis targeting with tumor homing peptides. Molecules, 23(5), 1190.

183. Smith, G. P. (1985). Filamentous fusion phage: Novel expression vectors that display cloned antigens on the virion surface. Science, 228, 1315-1317. https://doi.org/10.1126/science.4001944.

184. Song, S., Liu, D., Peng, J., et al. (2009). Novel peptide ligand directs liposomes toward EGF-R high-expressing cancer cells in vitro and in vivo. The FASEB Journal, 23, 1396-1404. https:// doi.org/10.1096/fj.08-117002.

185. Song, Y., Huang, Z., Xu, J., et al. (2014). Multimodal SPIONCREKA peptide based agents for molecular imaging of microthrombus in a rat myocardial ischemia-reperfusion model. Biomaterials, 35, 2961-2970. https://doi.org/10.1016/j. biomaterials.2013.12.038.

186. Spenlé, C., Saupe, F., Midwood, K., et al. (2015). Tenascin-C: Exploitation and collateral damage in cancer management. Cell Adhesion \& Migration, 9, 141-153. https://doi.org/10.1080/1933 6918.2014.1000074.

187. Spicer, C. D., Jumeaux, C., Gupta, B., \& Stevens, M. M. (2018). Peptide and protein nanoparticle conjugates: Versatile platforms for biomedical applications. Chemical Society Reviews, 47, 3574-3620.

188. Sugahara, K. N., Teesalu, T., Karmali, P. P., et al. (2009). Tissuepenetrating delivery of compounds and nanoparticles into tumors. Cancer Cell, 16, 510-520. https://doi.org/10.1016/j. ccr.2009.10.013.

189. Sugahara, K. N., Teesalu, T., Karmali, P. P., et al. (2010). Coadministration of a tumor-penetrating peptide enhances the efficacy of cancer drugs. Science, 328, 1031-1035. https://doi. org/10.1126/science. 1183057.

190. Sun, X., Li, Y., Liu, T., et al. (2017). Peptide-based imaging agents for cancer detection. Advanced Drug Delivery Reviews, 110-111, $38-51$

191. Tajrishi, M. M., Tuteja, R., \& Tuteja, N. (2011). Nucleolin: The most abundant multifunctional phosphoprotein of nucleolus. Communicative \& Integrative Biology, 4, 267-275.

192. Teesalu, T., Sugahara, K. N., Kotamraju, V. R., \& Ruoslahti, E. (2009). C-end rule peptides mediate neuropilin-1-dependent cell, vascular, and tissue penetration. Proceedings of the National Academy of Sciences of the United States of America, 106, 1615716162. https://doi.org/10.1073/pnas.0908201106.

193. Teesalu, T., Sugahara, K. N., \& Ruoslahti, E. (2013). Tumorpenetrating peptides. Frontiers in Oncology, 3, 216. https://doi. org/10.3389/fonc.2013.00216.

194. Teesalu, T., Sugahara, K. N., \& Ruoslahti, E. (2012). Mapping of vascular ZIP codes by phage display. Methods in Enzymology, 503, 35-56. https://doi.org/10.1016/B978-0-12-396962-0.00002-1.

195. Tetz, G., \& Tetz, V. (2018). Bacteriophages as new human viral pathogens. Microorganisms, 6, 54. https://doi.org/10.3390/ microorganisms6020054.
196. Tong, R., \& Langer, R. (2015). Nanomedicines targeting the tumor microenvironment. The Cancer Journal, 21, 314-321.

197. Usmani, S. S., Bedi, G., Samuel, J. S., et al. (2017). THPdb: Database of FDA-approved peptide and protein therapeutics. PLoS One, 12, e0181748. https://doi.org/10.1371/journal. pone. 0181748 .

198. Valetti, S., Maione, F., Mura, S., et al. (2014). Peptidefunctionalized nanoparticles for selective targeting of pancreatic tumor. Journal of Controlled Release, 192, 29-39. https://doi. org/10.1016/j.jconrel.2014.06.039.

199. Ventola, C. L. (2017). Progress in nanomedicine: Approved and investigational nanodrugs. Pharmacy and Therapeutics, 42, $742-755$.

200. Vrettos, E. I., Mező, G., \& Tzakos, A. G. (2018). On the design principles of peptide-drug conjugates for targeted drug delivery to the malignant tumor site. Beilstein Journal of Organic Chemistry, 14, 930-954. https://doi.org/10.3762/bjoc.14.80.

201. Wan, X. M., Chen, Y. P., Xu, W. R., et al. (2009). Identification of nose-to-brain homing peptide through phage display. Peptides, 30 , 343-350. https://doi.org/10.1016/j.peptides.2008.09.026.

202. Weber, W. A. (2009). Assessing tumor response to therapy. Journal of Nuclear Medicine, 50, 1S-10S. https://doi.org/10.2967/ jnumed.108.057174.

203. Werle, M., \& Bernkop-Schnürch, A. (2006). Strategies to improve plasma half life time of peptide and protein drugs. Amino Acids, 30, 351-367.

204. Wickström, M., Larsson, R., Nygren, P., \& Gullbo, J. (2011). Aminopeptidase N (CD13) as a target for cancer chemotherapy. Cancer Science, 102, 501-508. https://doi. org/10.1111/j.1349-7006.2010.01826.x.

205. Wyckoff, J., Wang, W., Lin, E. Y., et al. (2004). A paracrine loop between tumor cells and macrophages is required for tumor cell migration in mammary tumors. Cancer Research, 64, 7022-7029. https://doi.org/10.1158/0008-5472.CAN-04-1449.

206. Yan, J., He, W., Yan, S., et al. (2018). Self-assembled peptidelanthanide nanoclusters for safe tumor therapy: Overcoming and utilizing biological barriers to peptide drug delivery. ACS Nano, 12, 2017-2026. https://doi.org/10.1021/acsnano.8b00081.

207. Yan, L., Rosen, N., \& Arteaga, C. (2011). Targeted cancer therapies. Chinese Journal of Cancer, 30, 1-4. https://doi.org/10.5732/ CJC.010.10553.

208. Ye, F., Wu, X., Jeong, E.-K., et al. (2008). A peptide targeted contrast agent specific to fibrin-fibronectin complexes for cancer molecular imaging with MRI. Bioconjugate Chemistry, 19, 23002303. https://doi.org/10.1021/bc800211r.

209. Yin, H., Zhang, Q., Yang, J., et al. (2017). IRGD as a tumorpenetrating peptide for cancer therapy (Review). Molecular Medicine Reports, 15, 2925-2930.

210. Yoo, J., Park, C., Yi, G., et al. (2019). Active targeting strategies using biological ligands for nanoparticle drug delivery systems. Cancers (Basel), 11(5), 640. https://doi.org/10.3390/ cancers 11050640.

211. Yu, J., Sun, L., Zhou, J., et al. (2017). Self-assembled tumorpenetrating peptide-modified poly(l- $\gamma$-glutamylglutamine)paclitaxel nanoparticles based on hydrophobic interaction for the treatment of glioblastoma. Bioconjugate Chemistry, 28, 28232831. https://doi.org/10.1021/acs.bioconjchem.7b00519.

212. Zhang, B., Shen, S., Liao, Z., et al. (2014). Targeting fibronectins of glioma extracellular matrix by CLT1 peptide-conjugated nanoparticles. Biomaterials, 35, 4088-4098. https://doi.org/10.1016/j. biomaterials.2014.01.046.

213. Zhang, J., Niu, G., Lang, L., et al. (2017a). Clinical translation of a dual integrin $\alpha \mathrm{v} \beta 3$ - and gastrin-releasing peptide receptor-targeting PET radiotracer, 68Ga-BBN-RGD. Journal of Nuclear Medicine, 58, 228-234. https://doi.org/10.2967/jnumed.116.177048. 
214. Zhang, Y., Sun, T., \& Jiang, C. (2018). Biomacromolecules as carriers in drug delivery and tissue engineering. Acta Pharmaceutica Sinica $B, 8,34-50$.

215. Zhang, Y., Wei, J., Liu, S., et al. (2017b). Inhibition of platelet function using liposomal nanoparticles blocks tumor metastasis. Theranostics, 7, 1062-1071. https://doi.org/10.7150/thno.17908.

216. Zhang, Y., Yang, M., Park, J. H., et al. (2009). A surface-charge study on cellular-uptake behavior of F3- peptide-conjugated iron oxide nanoparticles. Small, 5, 1990-1996. https://doi.org/10.1002/ smll.200900520.

217. Zhao, N., Qin, Y., Liu, H., \& Cheng, Z. (2018). Tumor-targeting peptides: Ligands for molecular imaging and therapy. Anti-Cancer Agents in Medicinal Chemistry, 18, 74-86. https://doi.org/10.2174 /1871520617666170419143459.
218. Zhong, Y., Zhang, Y., Xu, J., et al. (2019). Low-intensity focused ultrasound-responsive phase-transitional nanoparticles for thrombolysis without vascular damage: A synergistic nonpharmaceutical strategy. ACS Nano, 13, 3387-3403. https://doi.org/10.1021/ acsnano.8b09277.

219. Zhou, J. E., Yu, J., Gao, L., et al. (2017). iNGR-modified liposomes for tumor vascular targeting and tumor tissue penetrating delivery in the treatment of glioblastoma. Molecular Pharmaceutics, 14, 1811-1820. https://doi.org/10.1021/acs. molpharmaceut.7b00101.

220. Zuo, H. (2019). IRGD: A promising peptide for cancer imaging and a potential therapeutic agent for various cancers. Journal of Oncology, 2019, 9367845. https://doi. org/10.1155/2019/9367845. 